\title{
Estimação de cópulas via ondaletas
}

\author{
Francyelle de Lima e Silva
}

TESE APRESENTADA

$\mathrm{AO}$

Instituto De Matemática e Estatística

DA

Universidade de São Paulo

PARA

OBTENÇÃO DO TÍTULO

$\mathrm{DE}$

Doutora EM CIÊNCIAS

\author{
Programa: Estatística \\ Orientador: Prof. Dr. Pedro Alberto Morettin \\ Coorientadora: Prof. ${ }^{a}$ Dr. ${ }^{a}$ Clélia Maria de Castro Toloi
}

Este trabalho foi financiado pela CAPES e CNPq 


\section{Estimação de cópulas via ondaletas}

Este exemplar correponde a redação final da tese devidamente corrigida e defendida por Francyelle de Lima e Silva, aprovada pela Comissão Julgadora.

Comissão Julgadora:

- Prof. Dr. Pedro Alberto Morettin (orientador) - IME-USP

- Prof ${ }^{\mathrm{a}}$. Dr ${ }^{\mathrm{a}}$. Clélia Maria de Castro Toloi (coorientadora) - IME-USP.

- Prof. Dr. Aluíso de Souza Pinheiro - IMECC-UNICAMP.

- Prof. Dr. João Ricardo Sato - UFABC.

- Prof $^{\mathrm{a}}$. Dr ${ }^{\mathrm{a}}$. Silvia Regina Costa Lopes - UFRGS. 


\section{Agradecimentos}

Aos meus pais, Ionice e Aparecido pelo incentivo, apoio e pelo amor incondicional. Meus melhores exemplos de vida.

Aos professores Clélia Maria de Castro Toloi e Pedro Alberto Morettin pelos ensinamentos.

Ao meu amado esposo Aldo Medina pelo companheirismo, amizade, apoio e pelo seu amor. Sou uma pessoa bem mais feliz e realizada ao lado dele.

Ao meu amado sobrinho Frederico Eduardo, pelo seu carinho. Seu amor incondicional me deu força pra superar cada obstáculo. Ele, com certeza, é um dos grandes amores da minha vida.

Ao meu grande amigo Jorge Torrejón pelas valiosas discussões probabilísticas, que possibilitaram alguns desenvolvimentos teóricos do trabalho. Agradeço também a Javier Hernándes pelas dicas e discussões.

A Rosemari Damasceno pelos oito anos de convívio em São Paulo, compartilhando cada momento de alegria, realização e de dificuldade. Foi um aprendizado valioso ter convivido tantos anos juntas.

Aos meus tios queridos Hilda e Gilberto, por me adotarem como sobrinha em todos esses anos. Eu os admiro e os respeito cada dia mais.

As minhas primas Ariadne e Gleice pelo carinho. Vocês são pessoas incríveis.

Aos meus queridos amigos Tiago Vargas, Dylene Agda, Gilberto Sassi, João Italo, Gustavo Prado e Alice Lemos por tornarem meus dias mais alegres durante o doutorado. A amizade de vocês é eterna. 
As minhas famílias de Caarapó e do Perú, pelo enorme carinho.

As secretárias do IME-USP Rosemeire A. de Oliveira, Tamara Cury, Cecilia Maria, Celia Pires e Rosana Benedetti, pelo profissionalismo, amizade e disponibilidade.

A todas as pessoas que ajudaram direta e indiretamente na elaboração deste trabalho.

A CAPES e a CNPq pelo apoio financeiro. 


\section{Resumo}

Cópulas tem se tornado uma importante ferramenta para descrever e analisar a estrutura de dependência entre variáveis aleatórias e processos estocásticos. Recentemente, surgiram alguns métodos de estimação não paramétricos, utilizando kernels e ondaletas.

Neste contexto, sabendo que cópulas podem ser escritas como expansão em ondaletas, foi proposto um estimador não paramétrico via ondaletas para a função cópula para dados independentes e de séries temporais, considerando processos $\alpha$-mixing.

Este estimador tem como característica principal estimar diretamente a função cópula, sem fazer suposição alguma sobre a distribuição dos dados e sem ajustes prévios de modelos ARMA - GARCH, como é feito em ajuste paramétrico para cópulas.

Foram calculadas taxas de convergência para o estimador proposto em ambos os casos, mostrando sua consistência. Foram feitos também alguns estudos de simulação, além de aplicações a dados reais.

Palavras-chave: Cópula, Estimação não paramétrica, Ondaletas, Processos $\alpha$-mixing 


\section{Abstract}

Copulas are important tools for describing the dependence structure between random variables and stochastic processes. Recently some nonparametric estimation procedures have appeared, using kernels and wavelets.

In this context, knowing that a copula function can be expanded in a wavelet basis, we have proposed a nonparametric copula estimation procedure through wavelets for independent data and times series under $\alpha$-mixing condition.

The main feature of this estimator is the copula function estimation without assumptions about the data distribution and without ARMA - GARCH modeling, like in parametric copula estimation.

Convergence rates for the estimator were computed, showing the estimator consistency. Some simulation studies were made, as well as analysis of real data sets.

Keywords: Copula, Nonparametric estimation, Wavelets, $\alpha$-mixing processes. 


\section{Sumário}

Lista de Figuras $\quad$ ix

Lista de Tabelas $\quad$ xi

1 Introdução 1

2 Conceitos básicos 3

2.1 Cópulas . . . . . . . . . . . . . . . . . . . . . . 3

2.2 Ondaletas . . . . . . . . . . . . . . . . . . 5

2.2.1 Análise de Multirresolução . . . . . . . . . . . . . . . 5

2.2.2 Ondaletas bidimensionais . . . . . . . . . . . . . . . 8

2.2 .3 Ondaletas periódicas . . . . . . . . . . . . . . . . . 11

2.3 Espaços de Besov . . . . . . . . . . . . . . . . . . . . . . . . . 12

2.4 Mixing . . . . . . . . . . . . . . . . . . . 15

2.5 Ergodicidade . . . . . . . . . . . . . . . . . 15

3 Estimação da Cópula $\quad 17$

3.1 Introdução . . . . . . . . . . . . . . . . . . . . . . 17

3.2 Estimação para o caso iid . . . . . . . . . . . . . . . 20

4 Estimação para o caso de séries temporais 31 
5 Simulações $\quad 41$

5.1 Simulação para o caso de séries temporais . . . . . . . . . . . . . . . 41

5.2 Simulação para o caso de séries temporais com threshold . . . . . . . 49

5.3 Simulação para o caso iid . . . . . . . . . . . . . . . . . . . 53

5.4 Simulações adicionais . . . . . . . . . . . . . . . 56

6 Aplicações $\quad 59$

6.1 Casos de séries temporais . . . . . . . . . . . . . . . . . 59

6.1.1 Séries CAC40 - DAX30 . . . . . . . . . . . 59

6.1 .2 Séries Ibovespa - IPC . . . . . . . . . . . . . . . . . 65

6.1 .3 Séries S\&P500 - DJIA . . . . . . . . . . . . 69

6.2 Caso iid . . . . . . . . . . . . . . . . . . . 72

6.2.1 Séries Lucro líquido - Margem de vendas . . . . . . . . . . . 73

6.3 Comentários . . . . . . . . . . . . . . . . . . 76

$\begin{array}{lll}7 & \text { Conclusões } & 77\end{array}$

$\begin{array}{ll}\text { A Desigualdades } & 81\end{array}$

A.1 Desigualdade de Dvoretsky-Kiefer-Wolfowitz . . . . . . . . . . . . 81

A.2 Desigualdade de Rosenthal . . . . . . . . . . . . . . . . . . . . . . 81

A.3 Desigualdade de Davydov . . . . . . . . . . . . . . . . . 82

B Ajuste de Modelos $\quad 83$

B.1 Casos de séries temporais . . . . . . . . . . . . . . 83

B.1.1 Séries CAC40 - DAX30 . . . . . . . . . . . 83

B.1.2 Séries Ibovespa - IPC . . . . . . . . . . . . . . . . . 87

B.1.3 Séries SP500 - DJ . . . . . . . . . . . . . . . 91

B.2 Caso independente . . . . . . . . . . . . . . . . . . . . 95

B.2.1 Séries Lucro líquido - Margem de vendas . . . . . . . . . . . . 95

$\begin{array}{ll}\text { Referências Bibliográficas } & 99\end{array}$ 


\section{Lista de Figuras}

5.1 Gráficos do Viés e do EQM. . . . . . . . . . . . . . . . . . . . . . . 45

5.2 Gráficos das Cópulas estimadas em vários níveis. . . . . . . . . . . . . . . . . 46

5.3 Gráficos das Cópulas estimadas em vários níveis para o componentes dependentes. 49

5.4 Gráficos das Cópulas estimadas em vários níveis para o componentes dependentes com Threshold. . . . . . . . . . . . . . . . . . . . 52

6.1 Gráficos dos log-retornos das séries. . . . . . . . . . . . . . . . . . . . . . . 60

6.2 Gráfico de dispersão entre as séries CAC40 e DAX30. . . . . . . . . . . . . . 60

6.3 Gráficos da cópula ajustada via ondaletas e das curvas de nível . . . . . . . . . 61

6.4 Gráficos da cópula ajustada e das curvas de nível - t-Student . . . . . . . . . . 62

6.5 Gráfico das estimativas para as medidas de dependência nas caudas . . . . . . . 65

6.6 Gráficos dos log-retornos das séries. . . . . . . . . . . . . . . . . . . . 65

6.7 Gráfico de dispersão entre as séries Ibovespa e IPC. . . . . . . . . . . . . . . 66

6.8 Gráficos da cópula estimada e das curvas de nível para as séries Ibovespa e IPC via ondaletas. . . . . . . . . . . . . . . . . . . . . . . 67

6.9 Gráficos da cópula estimada t-Student e das curvas de nível para séries Ibovespa IPC . . . . . . . . . . . . . . . . . . 67

6.10 Gráfico das estimativas para as medidas de dependência nas caudas para as séries Ibovespa e IPC . . . . . . . . . . . . . . . . . . . . . . . . . . 68

6.11 Gráficos dos log-retornos das séries. . . . . . . . . . . . . . . . . . . . 69

6.12 Gráfico de dispersão entre as séries S\&P500 e DJIA. . . . . . . . . . . . . . . 69 
6.13 Gráficos da cópula ajustada e das curvas de nível para as séries S\&P500 e DJIA via ondaletas. . . . . . . . . . . . . . . . . . . . 70 70

6.14 Gráficos da cópula t-Student ajustada e das curvas de nível para séries S\&P500 DJIA. . . . . . . . . . . . . . . . . . 71

6.15 Gráfico das estimativas para as medidas de dependência nas caudas para as séries S\&P500 e DJIA. . . . . . . . . . . . . . . . . . . . . . 72

6.16 Gráfico de dispersão entre os postos padronizados das séries Lucro líquido e Margem de vendas. . . . . . . . . . . . . . . . . . . . . . 73

6.17 Gráficos da cópula ajustada via ondaletas e das curvas de nível . . . . . . . . . 74

6.18 Gráfico das estimativas para as medidas de dependência nas caudas para as séries lucro líquido e margem de venda. . . . . . . . . . . . . . . . . . . . . . . 74

6.19 Gráfico das estimativas para as medidas de dependência nas caudas para as séries lucro líquido e margem de venda. . . . . . . . . . . . . . . . . . 


\section{Lista de Tabelas}

5.1 Média, Viés e EQM do estimador proposto utilizando Daubechie "D2" - componentes independentes, com $\mathrm{n}=1024$. . . . . . . . . . . . . . . 44

5.2 Média, Viés e EQM do estimador proposto utilizando Daubechie "D2" - componentes dependentes. . . . . . . . . . . . . . .

5.3 Média, Viés e EQM do estimador proposto utilizando Daubechie "D2" - caso dependente com threshold de quantil com $p=0.9$. . . . . . .

5.4 Média, Viés e EQM do estimador proposto utilizando Daubechie "D2" - caso iid com componentes independentes. . . . . . . . . . . . .

5.5 Média, Viés e EQM do estimador proposto utilizando Daubechie "D2" - caso iid com componentes dependentes. . . . . . . . . . . . . . . . 55

5.6 Média e desvio padrão do erro relativo entre as cópulas estimadas e as cópulas verdadeiras. . . . . . . . . . . . . . . . . . . 57

5.7 Média e desvio padrão do erro relativo entre as cópulas estimadas e as cópulas verdadeiras. . . . . . . . . . . . . . . . . . . . . . 58

6.1 Erro e ER entre as cópulas estimadas. . . . . . . . . . . . . . . . . 63

6.2 Erro e ER entre as cópulas estimadas para as séries Ibovespa - IPC. . 68

6.3 Erro e ER entre as cópulas ajustadas para as séries S\&P500 - DJIA. . 71

6.4 Erro e ER entre as cópulas estimadas para as séries lucro líquido margem de venda. . . . . . . . . . . . . . . . . . . . 75 


\section{Capítulo 1}

\section{Introdução}

Estruturas de dependência entre duas ou mais variáveis aleatórias tem sido amplamente estudadas visando descrever e detalhar de forma mais precisa como duas variáveis se comportam conjuntamente e como uma pode influenciar no comportamento da outra.

Visando estudar não só uma medida quantitativa de dependência, as cópulas surgem como uma alternativa ao estudo da dependência entre variáveis aleatórias.

Alguns métodos de estimação foram propostos, como por exemplo Fermanian and Scaillet (2003), que utiliza kernels para estimação das densidades marginais, as funções de distribuição e seus quantis e finalmente a função cópula. Morettin et al. (2011) propuseram um estimador com os mesmos passos, porém utilizando ondaletas.

Já Morettin et al. (2010) apresentaram um estimador que estima a cópula diretamente utilizando cópula empírica, porém sem apresentar as propriedades do estimador.

Como comentado em Morettin et al. (2010) e Morettin et al. (2011), os estimadores para cópula via ondaletas, apresentaram melhores resultados em termos de Viés e Erro quadrático médio, quando comparados a outros estimadores não paramétricos, como por exemplo os estimadores via kernels.

Com o intuito de obter um estimador consistente, neste trabalho, assim como su- 
gerido em Morettin et al. (2010), foi proposto a estimação da função cópula por meio da expansão direta em bases de ondaletas. Os coeficientes, sob suposições adequadas, são estimados diretamente utilizando algoritmo piramidal no caso bidimensional.

No Capítulo 2, são apresentados alguns conceitos e definições, que facilitam a leitura e compreensão do trabalho.

As idéias fundamentais do processo de estimação para o caso iid podem ser vistas no Capítulo 3. Ao final, também apresenta-se a taxa de convergência do estimador, a um nível $l^{*}$ ótimo.

A estimação para o caso dependente, considerando um processo $\alpha$-mixing é apresentada no Capítulo 4.

No Capítulo 5, são apresentados os resultados obtidos por meio de simulações para o caso iid, e para o caso dependente.

As aplicações são apresentadas no Capítulo 6. Foram ajustadas cópulas via ondaletas para as séries CAC40, da França e DAX30, da Alemanha, visando analisar se os índices estão correlacionados ou não. Também foi feito ajuste para as séries Ibovespa e IPC do México, e para as séries americanas S\&P500 e DJIA. Para fins de comparação, foram ajustados modelos ARMA-GARCH para todas as séries, e aos resíduos padronizados foram ajustadas as cópulas paramétricas Normal, t-Student, Gumbel, Clayton e Frank. Após os ajustes, foram calculadas medidas que indicam o quanto as cópulas ajustadas via ondaletas, utilizando somente função escala e sem suposições sobre o comportamento dos dados, diferem dos modelos paramétricos.

As conclusões e alguns comentários, assim como algumas idéias sobre pesquisas futuras são apresentados no Capítulo 7. 


\section{Capítulo 2}

\section{Conceitos básicos}

Neste capítulo serão apresentados alguns conceitos e definições utilizados ao longo do trabalho.

\subsection{Cópulas}

As definições, para melhor compreensão, foram feitas para o caso bidimensional, porém podem ser estendidas para o caso multidimensional.

Sejam $X$ e $Y$ variáveis aleatórias com função distribuição conjunta

$$
H(x ; y)=P(X \leq x ; Y \leq y),
$$

e marginais contínuas $F($.$) e G($.$) , respectivamente. Suponha que se transforme o$ vetor aleatório $(X ; Y)$ componente a componente, para que ele tenha distribuições marginais $U(0,1)$. Essa transformação pode ser obtida usando a transformada integral de probabilidade:

$$
T: \mathbb{R}^{2} \mapsto \mathbb{R}^{2},(x ; y) \mapsto(F(x) ; G(y))
$$

A função distribuição conjunta $C(. ;$. $)$ de $(F(x) ; G(y))$ é então chamada cópula do vetor aleatório $(X ; Y)$, ou equivalentemente, cópula associada à distribuição conjunta 
$H(. ;$.$) . Logo, segue que$

$$
H(x ; y)=P(F(X) \leq F(x) ; G(Y) \leq G(y))=C(F(x) ; G(y)) .
$$

Um resultado, muito importante em teoria de cópulas, que dá existência e unicidade da função $C(. ;$.) é o Teorema de Sklar.

Teorema 2.1: Seja H(.;.) uma função de distribuição conjunta com marginais $F($.$) e G($.$) . Então, existe uma cópula bidimensional C(. ;$.$) , tal que:$

$$
H(x ; y)=C(F(x) ; G(y))
$$

Se $F(),. G($.$) são contínuas, então C(. ;$.$) é única, caso contrário C(. ;$.$) é univoca-$ mente determinada no conjunto $I M(F) \times I M(G)$, onde $I M(F)$ representa o conjunto imagem de $F($.$) .$

Reciprocamente, se $C(. ;$.) é uma cópula bidimensional e $F(),. G($.$) são funções de$ distribuição, então a função H(.;.), definida em (2.1) é uma função de distribuição conjunta bidimensional.

Considerando a cópula como uma função definida em (2.1), considera-se o seguinte teorema, enunciado em Nelsen (2005) p.14, como Teorema 2.2.8:

Teorema 2.2: Seja C(.;.) uma cópula. Se $\frac{\partial}{\partial v} C(u ; v)$ e $\frac{\partial^{2}}{\partial u \partial v} C(u ; v)$ são contínuas em $[0,1]^{2}$ e $\frac{\partial}{\partial u} C(u ; v)$ existe para todo $u \in[0,1]$ quando $v=0$, então $\frac{\partial}{\partial u} C(u ; v)$ e $\frac{\partial^{2}}{\partial u \partial v} C(u ; v)$ existem em $[0,1]^{2}$, e $\frac{\partial^{2}}{\partial u \partial v} C(u ; v)=\frac{\partial^{2}}{\partial v \partial u} C(u ; v)$.

Demonstração: Veja Seeley (1961).

Assim, supondo válido o Teorema 2.2, ou seja, que existem as derivadas parciais de $C(u ; v)$, a densidade cópula é dada por

$$
c(u ; v)=\frac{\partial^{2}}{\partial u \partial v} C(u ; v) .
$$




\subsection{Ondaletas}

De acordo com Morettin (2014), ondaletas são funções que satisfazem certas propriedades, localizadas no tempo e no espaço. Elas podem ser suaves ou não, simétricas ou não e podem ter expressões matemáticas simples ou não.

Considere o espaço $L^{2}(\mathbb{R})$ de todas as funções de quadrado integrável. A idéia do uso de ondaletas é gerar $L^{2}(\mathbb{R})$ a partir de funções $\phi$ (ondaleta pai ou função de escala) e $\psi$ (ondaleta mãe) através de dilatações(compressões) e translações, do tipo:

$$
\begin{aligned}
& \phi_{j, k}(t)=2^{\frac{j}{2}} \phi\left(2^{j} t-k\right), j, k \in \mathbb{Z}, \\
& \psi_{j, k}(t)=2^{\frac{j}{2}} \psi\left(2^{j} t-k\right), j, k \in \mathbb{Z}
\end{aligned}
$$

de tal forma que possamos aproximar qualquer função pertencente a $L^{2}(\mathbb{R})$ por meio de alguma base, de forma precisa.

Sendo assim, será definida a análise de multirresolução, que na verdade é uma sequência de espaços fechados de $L^{2}(\mathbb{R})$, que apresentam boas propriedades de aproximação.

\subsubsection{Análise de Multirresolução}

Seja $\phi($.$) pertencente a L^{2}(\mathbb{R})$, de tal forma que suas translações $\left\{\phi_{0 k}, k \in \mathbb{Z}\right\}=$ $\{\phi(.-k), k \in \mathbb{Z}\}$ formam um sistema ortonormal(SO).

De acordo com as dilatações e translações apresentadas na equação (2.2), definemse os espaços lineares encaixados

$$
\ldots V_{-2} \subset V_{-1} \subset V_{0} \subset V_{1} \subset V_{2} \subset \ldots \subset L^{2}(\mathbb{R})
$$

em que 


$$
\begin{aligned}
V_{0} & =\left\{f(x)=\sum_{k} c_{k} \phi(x-k): \sum_{k}\left|c_{k}\right|^{2}<\infty\right\} \\
V_{1} & =\left\{h(x)=f(2 x): f \in V_{0}\right\} \\
\vdots & \\
V_{j} & =\left\{h(x)=f\left(2^{j} x\right): f \in V_{0}\right\}, j \in \mathbb{Z} .
\end{aligned}
$$

Uma aproximação por multirresolução de $L^{2}(\mathbb{R})$ é por definição uma sequência crescente de subespaços $V_{j}, j \in \mathbb{Z}$ de $L^{2}(\mathbb{R})$ de acordo com os espaços lineares definidos anteriormente, e também com as seguintes propriedades:

$$
\begin{aligned}
& \text { (1) } \bigcap_{-\infty}^{\infty} V_{j}=\{0\} \\
& \text { (2) } \bigcup_{-\infty}^{\infty} V_{j} \text { é denso em } L^{2}(\mathbb{R}) .
\end{aligned}
$$

Também, para cada $j$, há um complemento $W_{j}$ para $V_{j}$ em $V_{j+1}$, tal que

$$
V_{j+1}=V_{j} \oplus W_{j}
$$

Assim,

$$
\begin{aligned}
V_{j+1} & =\left(V_{j-1} \oplus W_{j-1}\right) \oplus W_{j}=\left(V_{j-2} \oplus W_{j-2}\right) \oplus W_{j-1} \oplus W_{j} \\
& =\ldots=V_{0} \oplus \bigoplus_{k=0}^{j} W_{k} .
\end{aligned}
$$

Pela propriedade (2), tem-se

$$
L^{2}(\mathbb{R})=V_{0} \oplus \bigoplus_{j=0}^{\infty} W_{j} .
$$

Mas note que pelas substituições sucessivas dadas em (2.4), o nível $j=0$ é arbitrário, ou seja, é possível escrever 


$$
L^{2}(\mathbb{R})=V_{j_{0}} \oplus \bigoplus_{j=j_{0}}^{\infty} W_{j}
$$

Considerando a análise de multirresolução(AMR), é possível obter a função $\psi$ a partir de $\phi$, em que $\left\{\psi_{0 k}, k \in \mathbb{Z}\right\}=\{\psi(.-k), k \in \mathbb{Z}\}$ é um SO em $W_{0}$ e, consequentemente, tem-se que $\psi_{j, k}(t)$ definida em (2.3) é um SO para $W_{j}$.

Logo, $\left\{\left\{\phi_{j_{0}, k}\right\},\left\{\psi_{j, k}\right\}, k \in \mathbb{Z}, j \geq j_{0}\right\}$ é um SO em $L^{2}(\mathbb{R})$.

Sendo assim, qualquer função $f \in L^{2}(\mathbb{R})$ pode ser representada por

$$
f(t)=\sum_{k} c_{j_{0}, k} \phi_{j_{0}, k}(t)+\sum_{j \geq j_{0}}^{\infty} \sum_{k} d_{j, k} \psi_{j, k}(t),
$$

em que $c_{j_{0}, k}$ e $d_{j, k}$ são os coeficientes de ondaletas.

Para que $f$ tenha uma única representação, em termos da convergência em $L^{2}$, os coeficientes são dados por

$$
c_{j_{0}, k}=\int_{\mathbb{R}} f(t) \phi_{j_{0}, k}(t) d t
$$

e

$$
d_{j, k}=\int_{\mathbb{R}} f(t) \psi_{j, k}(t) d t .
$$

Como consequência da expansão de uma função $f$ em bases de ondaletas, tem-se que uma forma de aproximar uma função $f$, é utilizar a projeção de $f$ em $V_{j+1}$ pelos subespaços $V_{j_{0}}$ e $W_{j}$, em que a projeção é dada por

$$
P_{V_{j+1}}(f)=\sum_{k} c_{j_{0}, k} \phi_{j_{0}, k}(t)+\sum_{j \geq j_{0}}^{\infty} \sum_{k} d_{j, k} \psi_{j, k}(t),
$$

em que $c_{j_{0}, k}$ e $d_{j, k}$ são definidos como em (2.5) e (2.6).

Agora, considerando uma função $f(x, y) \in L^{2}\left(\mathbb{R}^{2}\right)$, a seguir será mostrado como $f(x, y)$ também pode ser expandida em bases de ondaletas através de ondaletas bidimensionais. 


\subsubsection{Ondaletas bidimensionais}

Há duas formas de construir bases de ondaletas bidimensionais:

- Com uma única escala, a partir do produto tensorial de duas bases unidimensionais;

- Como o produto tensorial de duas bases unidimensionais, com escalas distintas.

Neste trabalho, utilizam-se bases com uma única escala, obtidas através do produto tensorial de bases $\left\{\phi_{l, k}, \psi_{j, k}(t), j \leq l, k \in \mathbb{Z}\right\}$.

Seja

$$
\ldots \mathbf{V}_{-2} \subset \mathbf{V}_{-1} \subset \mathbf{V}_{0} \subset \mathbf{V}_{1} \subset \mathbf{V}_{2} \subset \ldots \subset L^{2}\left(\mathbb{R}^{2}\right)
$$

uma análise de multirresolução bidimensional, em que

$$
\mathbf{V}_{j}=\bigotimes_{i=1}^{2} \mathbf{V}_{j,(i)}
$$

e considerando $\Phi(x ; y)=\phi(x) \phi(y)$ a função escala bidimensional, tem-se que $\left\{\Phi_{j, \mathbf{k}}(x ; y), \mathbf{k}=\left(k_{1}, k_{2}\right), \mathbf{k} \in \mathbb{Z}^{2}\right\}$ é uma base para $\mathbf{V}_{j}$.

Assim, pela AMR,

$$
\begin{aligned}
\mathbf{V}_{j+1} & =\mathbf{V}_{j+1,(1)} \oplus \mathbf{V}_{j+1,(2)} \\
& =\left(\mathbf{V}_{j,(1)} \oplus \mathbf{W}_{j,(1)}\right) \otimes\left(\mathbf{V}_{j,(2)} \oplus \mathbf{W}_{j,(2)}\right) \\
& =\left(\mathbf{V}_{j,(1)} \otimes \mathbf{V}_{j,(2)}\right) \oplus\left(\mathbf{V}_{j,(1)} \otimes \mathbf{W}_{j,(2)}\right) \otimes\left(\mathbf{W}_{j,(1)} \otimes \mathbf{V}_{j,(2)}\right) \otimes\left(\mathbf{W}_{j,(1)} \otimes \mathbf{W}_{j,(2)}\right) \\
& =\mathbf{V}_{j} \oplus \mathbf{W}_{j}^{(h)} \oplus \mathbf{W}_{j}^{(v)} \oplus \mathbf{W}_{j}^{(d)} .
\end{aligned}
$$

Os índices $\mu=\{h, v, d\}$ definem os espaços dos detalhes em três direções: horizontal, vertical e diagonal, em que $\mathbf{W}_{j}^{(h)}, \mathbf{W}_{j}^{(v)}$ e $\mathbf{W}_{j}^{(d)}$ são obtidos por meio das translações com respeito a dois argumentos $k_{1}$ e $k_{2}$ em 


$$
\begin{aligned}
\Psi_{j, \mathbf{k}}^{h}(x ; y) & =\phi_{j, k_{1}}(x) \psi_{j, k_{2}}(y), \\
\Psi_{j, \mathbf{k}}^{v}(x ; y) & =\psi_{j, k_{1}}(x) \phi_{j, k_{2}}(y), \\
\Psi_{j, \mathbf{k}}^{d}(x ; y) & =\psi_{j, k_{1}}(x) \psi_{j, k_{2}}(y) .
\end{aligned}
$$

Agora, considere

$$
\mathbf{V}_{j+1}=\mathbf{V}_{j} \oplus\left[\mathbf{W}_{j}^{(h)} \oplus \mathbf{W}_{j}^{(v)} \oplus \mathbf{W}_{j}^{(d)}\right]=\mathbf{V}_{j} \oplus \mathbf{W}_{j}^{*}
$$

em que $\mathbf{W}_{j}^{*}$ é o complemento ortogonal de $\mathbf{V}_{j}$ em $\mathbf{V}_{j+1}$.

Analogamente ao caso unidimensional, faz-se

$$
\begin{aligned}
\mathbf{V}_{j+1} & =\mathbf{V}_{j} \oplus \mathbf{W}_{j}^{*}=\left(\mathbf{V}_{j-1} \oplus \mathbf{W}_{j-1}^{*}\right) \oplus \mathbf{W}_{j}^{*}=\left(\mathbf{V}_{j-2} \oplus \mathbf{W}_{j-2}^{*}\right) \oplus \mathbf{W}_{j-1}^{*} \oplus \mathbf{W}_{j}^{*} \\
& =\ldots=\mathbf{V}_{j_{0}} \oplus \bigoplus_{m=j_{0}}^{j} \mathbf{W}_{m}^{*}=\mathbf{V}_{j_{0}} \oplus\left[\bigoplus_{m=j_{0}}^{j}\left(\mathbf{W}_{m}^{(h)} \oplus \mathbf{W}_{m}^{(v)} \oplus \mathbf{W}_{m}^{(d)}\right)\right] .
\end{aligned}
$$

Logo,

$$
\left\{\Phi_{l, \mathbf{k}}(x ; y), \mathbf{k}=\left(k_{1}, k_{2}\right)\right\}_{\mathbf{k}} \cup\left\{\Psi_{j, \mathbf{k}}^{\mu}(x ; y), \mathbf{k}=\left(k_{1}, k_{2}\right), \mu=h, v, d\right\}_{j \geq l, \mathbf{k}}
$$

é uma base para $L^{2}\left(\mathbb{R}^{2}\right)$, em que

$$
\begin{aligned}
\Phi_{l, \mathbf{k}}(x ; y) & =\phi_{l, k_{1}}(x) \phi_{l, k_{2}}(y), \\
\Psi_{j, \mathbf{k}}^{h}(x ; y) & =\phi_{j, k_{1}}(x) \psi_{j, k_{2}}(y), \\
\Psi_{j, \mathbf{k}}^{v}(x ; y) & =\psi_{j, k_{1}}(x) \phi_{j, k_{2}}(y), \\
\Psi_{j, \mathbf{k}}^{d}(x ; y) & =\psi_{j, k_{1}}(x) \psi_{j, k_{2}}(y) .
\end{aligned}
$$

Sendo assim, uma função pertencente a $L^{2}\left(\mathbb{R}^{2}\right)$ pode ser escrita como

$$
f(x ; y)=\sum_{\mathbf{k} \in \mathbb{Z}^{2}} c_{l, \mathbf{k}} \Phi_{l, \mathbf{k}}(x ; y)+\sum_{j \geq l}^{\infty} \sum_{\mathbf{k} \in \mathbb{Z}^{2}} \sum_{\mu=h, v, d} d_{j, \mathbf{k}}^{\mu} \Psi_{j, \mathbf{k}}^{\mu}(x ; y),
$$


com coeficientes dados por

$$
c_{l, \mathbf{k}}=\int_{\mathbb{R}^{2}} f(x ; y) \Phi_{l, \mathbf{k}}(x ; y) d x d y
$$

$\mathrm{e}$

$$
d_{j, \mathbf{k}}^{\mu}=\int_{\mathbb{R}^{2}} f(x ; y) \Psi_{j, \mathbf{k}}^{\mu}(x ; y) d x d y
$$

Neste caso, a transformada discreta de ondaletas, utilizando o algoritmo piramidal introduzido por Mallat (1989a) e Mallat (1989b), pode ser estendido para o caso bidimensional. Os coeficientes serão matrizes, que podem ser representadas como na Figura 2.1.

\begin{tabular}{|c|c|c|c|}
\hline$C_{j_{0}}$ & $D_{j_{0}}^{h}$ & \multirow{2}{*}{$D_{j_{0}+1}^{h}$} & \\
\cline { 1 - 2 }$D_{j_{0}}^{v}$ & $D_{j_{0}}^{d}$ & & \\
\hline$D_{j_{0}+1}^{v}$ & $D_{j_{0}+1}^{d}$ & \\
& & \\
& & \\
& & \\
& $D_{j_{0}+2}^{v}$ & $D_{j_{0}+2}^{d}$ \\
& & \\
\end{tabular}

Figura 2.1: Representação dos coeficientes das ondaletas bidimensionais

Ao se falar de aproximação de funções utilizando ondaletas, a principal questão a ser respondida é como seria possível calcular os coeficientes.

Em algumas situações há funções cujo domínio pertence ao espaço $L^{2}([0,1])$, e deseja-se utilizar ondaletas para aproximar essas funções. Porém, a única base de ondeletas em $[0,1]$ é a base de Haar. Para obter outras bases ortogonais em $L^{2}$, é necessário periodizá-las. 


\subsubsection{Ondaletas periódicas}

Considere as função de escala $\phi($.$) e a função de detalhes \psi($.$) . As ondaletas periódicas$ são definidas como

$$
\tilde{\phi}_{j, k}(x)=\sum_{n \in \mathbb{N}} \phi(x+n)
$$

$\mathrm{e}$

$$
\tilde{\psi}_{j, k}(x)=\sum_{n \in \mathbb{N}} \psi(x+n)
$$

As funções periodizadas também definem uma AMR sobre $L^{2}([0,1])$. Isto também pode ser extendido para o caso bidimensional e considerando o produto tensor, é possível obter uma base para $L^{2}\left([0,1]^{2}\right)$.

Alguns outros métodos para obter bases em ondaletas para funções em intervalos podem ser vistos em Cohen et al. (1993). Porém, no trabalho serão utilizadas as bases detalhadas em Strang and Nguyen (1996).

Alguns problemas poderão surgir neste caso, como por exemplo descontinuidade nas bordas. Visando contornar este problema, de acordo com Cohen et al. (1993), são propostos alguns métodos para correção do problema das bordas.

Simetrização: Este método consiste na replicação simétrica dos sinais ou imagens fora do suporte original;

Periodização: Assume que os sinais ou imagens podem ser replicados por extensão periódica;

Zero-padding: Assume que os sinais ou imagens tenham valor zero fora do suporte original.

Ao se falar de ondaletas, é importante destacar que alguns espaços de funções admitem caracterização em termos dos coeficientes de ondaletas, ou seja, se uma função pertence a um determinado espaço, e pode ser espandida em bases de ondaletas, os coeficientes admitem determinados comportamentos. 
Neste trabalho, utilizam-se as funções definidas em espaços de Besov, que apresentam ferramentas mais gerais que, por exemplo, os espaços de Sobolev e Holder.

\subsection{Espaços de Besov}

Como feito em Härdle et al. (1998), antes de definir os espaços de Besov, são definidos os espaços de Sobolev.

Inicialmente é necessário introduzir o conceito de diferenciabilidade fraca.

Proposição 2.1: Seja $D(\mathbb{R})$ o espaço de funções com suporte compacto infinitamente diferenciável, e seja $f$ uma função definida na reta real, integrável em todo intervalo limitado. As seguintes propriedades são equivalentes:

1. Existe uma função $g$ definida na reta real, integrável em todo intervalo limitado tal que

$$
\forall x \leq y, \int_{x}^{y} g(u) d u=f(y)-f(x)
$$

2. Existe uma função $g$ definida na reta real, integrável em todo intervalo limitado tal que

$$
\forall \varphi \in D(\mathbb{R}): \int f(u) \varphi^{\prime}(u) d u=-\int g(u) \varphi(u) d u .
$$

Definição 2.1: A função $f$ satisfazendo as propriedades da Proposição 2.1 é chamada fracamente diferenciável. A função $g$ é definida quase sempre, é chamada de derivada fraca de $f$ e é denotada por $f^{\prime}$.

Definição 2.2: Uma função $f$ é $N$ vezes fracamente diferenciável, se tem $N-1$ derivadas fracas fracamente diferenciável, implicando que as derivadas fracas $f, f^{\prime}, \ldots$, $f^{N-1}$ sejam contínuas.

Espaços de Sobolev: Seja $1 \leq p \leq \infty, m \in\{0,1, \ldots$,$\} . A função f \in L_{p}(\mathbb{R})$ pertence ao espaço de Sobolev $W_{p}^{m}(\mathbb{R})$, se é m-vezes fracamente diferenciável, e se $f^{(i)} \in L_{p}(\mathbb{R}), j=1,2, \ldots, m$. Em particular, $W_{p}^{0}(\mathbb{R})=L_{p}(\mathbb{R})$. 
O espaço $W_{p}^{m}(\mathbb{R})$ é naturalmente equipado com a norma

$$
\|f\|_{W_{p}^{m}}=\|f\|_{p}+\left\|f^{(m)}\right\|_{p}
$$

Módulo de Continuidade: seja $f$ uma função em $L_{p}(\mathbb{R}), 1 \leq p \leq \infty$. Seja $\tau_{h} f(x)=f(x-h), \Delta_{h} f=\tau_{h} f-f$. Define-se também $\Delta_{h}^{2} f=\Delta_{h} \Delta_{h} f$. Para $t \geq 0$, os modulos de continuidade são definidos por

$$
\omega_{p}^{1}(f, t)=\sup _{|h| \leq t}\left\|\Delta_{h} f\right\|_{p}, \omega_{p}^{2}(f, t)=\sup _{|h| \leq t}\left\|\Delta_{h}^{2} f\right\|_{p} .
$$

Espaços de Besov: Sejam $1 \leq p, q \leq \infty$, e $s=n+\alpha$, com $n \in\{0,1, \ldots\}$, e $0<\alpha \leq 1$. O espaço de Besov $\mathfrak{B}_{p}^{s, q}(\mathbb{R})$ é o espaço de todas as funções $f$, tal que

$$
f \in W_{p}^{n}(\mathbb{R}), \text { e } \omega_{p}^{2}\left(f^{(n)}, t\right)=\epsilon(t) t^{\alpha},
$$

para $t \in[0, \infty)$, e $\|\epsilon\|_{q}^{*}<\infty$ definida como

$$
\|\epsilon\|_{q}^{*}= \begin{cases}\left(\int_{0}^{\infty}|\epsilon(t)|^{q} \frac{d t}{t}\right)^{\frac{1}{q}} & \text { se } 1 \leq q<\infty \\ \operatorname{ess} \sup _{t}|\epsilon(t)| & \text { se } q=\infty\end{cases}
$$

Então, se $q<\infty,\|\cdot\|_{q}^{*}$ é uma norma no espaço $L^{q}$ ponderado $L^{q}\left([0, \infty), \frac{d t}{t}\right)$. Mas, se $q=\infty, \epsilon$ é dita essencialmente limitada e pertencente ao espaço $L^{\infty}([0, \infty))$ se existir uma constante $C \in \mathbb{R}$, tal que $\mu\{t \in[0, \infty):|\epsilon(t)|>C\}=0$. Sendo assim, a norma $\|\epsilon\|_{L^{\infty}([0, \infty))}^{*}$ é definida como

$$
\|\epsilon\|_{L^{\infty}([0, \infty))}^{*}=\inf \{C \in \mathbb{R}: \mu(\{t \in[0, \infty):|\epsilon(t)|>C\})=0\} .
$$

O espaço $\mathfrak{B}_{p}^{s, q}(\mathbb{R})$ é equipado com a norma

$$
\|f\|_{s p q}=\|f\|_{W_{p}^{n}}+\left\|\frac{\omega_{p}^{2}\left(f^{(n)}, t\right)}{t^{\alpha}}\right\|_{q}^{*} .
$$

Note que um espaço de Besov é uma generalização de um espaço de Sobolev, em que $W_{1}^{s}(\mathbb{R})=\mathfrak{B}_{1}^{s, 2}(\mathbb{R})$. 
Sendo assim, de acordo com Meyer (1992):

Proposição 2.2: Seja $\Lambda_{j}$ um conjunto de índices tais que $\left\{\psi_{i}, i \in \Lambda_{j}\right\}$ forma um SO para o subespaço $W_{j}$. Existem duas constantes $0<C \leq C^{\prime}$, tais que, para qualquer expoente $p \in[1, \infty]$, para cada $j \in \mathbb{Z}$ e para cada soma finita $f(x)=$ $\sum_{i \in \Lambda_{j}} d_{i} \psi_{i}(x)$ em $W_{j}$

$$
C\|f\|_{p} \leq 2^{\frac{j}{2}} 2^{\frac{-j}{p}}\left(\sum_{i \in \Lambda_{j}}\left|d_{i}\right|^{p}\right)^{\frac{1}{p}} \leq C^{\prime}\|f\|_{p} .
$$

Deste resultado, é possível obter a caracterização de espaços de Besov por meio dos coeficientes das ondaletas. Ou seja, a função $f=\sum_{k} c_{j_{0} k} \phi_{j_{0} k}+\sum_{j \geq j_{0}} \sum_{k} d_{j k} \psi_{j k}(x)$ pertence ao espaço de $\operatorname{Besov} \mathfrak{B}_{p}^{s, q}(\mathbb{R})$ se seus coeficientes satisfizerem as seguintes condições:

$$
\left(\sum_{k}\left|c_{j_{0} k}\right|^{p}\right)^{\frac{1}{p}}<\infty
$$

$\mathrm{e}$

$$
\left(\sum_{k}\left|d_{j k}\right|^{p}\right)^{\frac{1}{p}} \leq 2^{-j\left(s+\frac{1}{2}+\frac{1}{p}\right)} \epsilon_{j}
$$

em que $\epsilon_{j} \in l_{q}(\mathbb{N})$.

Logo, a partir da análise de multirresolução e dos espaços de Besov definidos anteriormente, é possível obter estimadores para as funções $f \in L^{2}(\mathbb{R})$ a partir de sua representação em bases de ondaletas, com boas propriedades assintóticas, não só em norma $L^{2}$, mas também em espaços funcionais como Besov.

Ao se falar de estimação de funções, tanto unidimensionais como bidimensionais, existem muitos resultados na literatura para o caso iid. Visando obter resultados mais abrangentes, este trabalho também apresenta resultados para o caso dependente, supondo estrutura mixing. 


\section{$2.4 \quad$ Mixing}

Exitem várias estruturas mixing definidas para processos em geral, como pode ser visto em Doukhan (1995). Neste trabalho, será utilizada a estrutura $\alpha$-mixing.

Medida $\alpha$-mixing: Seja $(\Omega, \mathscr{F}, \mathbb{P})$ um espaço de probabilidade. Dadas as $\sigma$ álgebras $\mathscr{A}$ e $\mathscr{B}$ em $(\Omega, \mathscr{F}, \mathbb{P})$, o coeficiente $\alpha$-mixing $\alpha(\mathscr{A}, \mathscr{B})$ é definido por

$$
\alpha(\mathscr{A}, \mathscr{B})=\sup _{(A, B) \in \mathscr{A} \times \mathscr{B}}|\mathbb{P}(A \cap B)-\mathbb{P}(A) \mathbb{P}(B)|
$$

Já para um processo estacionário $\left\{X_{t}, t \in T\right\}$, seja $\mathscr{F}_{k}$ a $\sigma$-álgebra gerada por $\left\{X_{t}, t \leq k\right\}$, e $\mathscr{F}_{k+h}$ a $\sigma$-álgebra gerada por $\left\{X_{t}, t \geq k+h\right\}$. O coeficiente $\alpha$-mixing é definido por

$$
\alpha(h)=\sup _{(A, B) \in \mathscr{F}_{k} \times \mathscr{F}_{k+h}}|\mathbb{P}(A \cap B)-\mathbb{P}(A) \mathbb{P}(B)|
$$

\subsection{Ergodicidade}

Considere o processo mixing definido sob o espaço de probabilidade dado anteriormente, e considere uma transformação $T$. A definição de processo mixing também é dada por

$$
\lim _{t \rightarrow \infty}\left|\mathbb{P}\left(A \cap T^{-t}(B)\right)-\mathbb{P}(A) \mathbb{P}\left(T^{-t}(B)\right)\right|=0
$$

Se $\mathbb{P}$ é $T$-invariante, mixing é equivalente a

$$
\lim _{t \rightarrow \infty} \mathbb{P}\left(A \cap T^{-t}(B)\right)=\mathbb{P}(A) \mathbb{P}(B) .
$$

Logo como consequência, tem-se que mixing implica ergodicidade, pois uma medida $T$-invariante é ergodica se para todo evento $A \in \mathscr{F}, \operatorname{com} T^{-1}(A)=A, \mathbb{P}(A)=0$ ou $\mathbb{P}(A)=1$. 


\section{Capítulo 3}

\section{Estimação da Cópula}

\subsection{Introdução}

Considerando a estimação paramétrica de cópulas, para dados iid, é necessário a escolha prévia de uma família de cópulas, fazer vários ajustes e depois escolher o mais adequado. Para séries temporais, a amostra não é composta de pares independentes, logo é necessário fazer ajustes preliminares de modelos ARMA-GARCH para cada série, obter os resíduos padronizados e a esses resíduos, ajustar cópulas paramétricas, e então escolher a mais adequada.

Considerando um método não paramétrico, teoria de ondaletas tem sido considerada como uma ferramenta eficiente para estimação de densidades, funções e também cópulas, fornecendo bons resultados.

Como mencionado no capítulo anterior, funções pertencentes a $L^{2}(\mathbb{R})$ podem ser expandidas em bases de ondaletas, e então estimadas por meio dos coeficientes das ondaletas.

Neste contexto, considere uma cópula $C(u ; v)$ desconhecida e uma base adequada em $L^{2}\left([0,1]^{2}\right)$. 
A cópula $C(u ; v)$ pode ser escrita como

$$
C(u ; v)=\sum_{\mathbf{k}} c_{l, \mathbf{k}} \Phi_{l, \mathbf{k}}(u ; v)+\sum_{j \geq l}^{\infty} \sum_{\mathbf{k} \in \mathbb{Z}^{2}} \sum_{\mu=h, v, d} d_{j, \mathbf{k}}^{\mu} \Psi_{j, \mathbf{k}}^{\mu}(u ; v),
$$

em que $c_{l, \mathbf{k}}=\int_{[0,1]^{2}} C(u ; v) \Phi_{l, \mathbf{k}}(u ; v) d u d v$ e $d_{j, \mathbf{k}}^{\mu}=\int_{[0,1]^{2}} C(u ; v) \Psi_{j, \mathbf{k}}^{\mu}(u ; v) d u d v$.

Neste caso, a cópula pode ser estimada diretamente através da estimação dos coeficientes, sem necessariamente, dada uma amostra de $X$ e $Y$, estimar as densidades $f_{X}$ e $g_{Y}$, as funções de distribuição $F($.$) e G($.$) , além da conjunta H(F(.) ; G()$.$) , como$ proposto em Morettin et al. (2011).

Assim,

$$
\hat{C}(u ; v)=\sum_{\mathbf{k}} \hat{c}_{l, \mathbf{k}} \Phi_{l, \mathbf{k}}(u ; v)+\sum_{j \geq l}^{J} \sum_{\mathbf{k} \in \mathbb{Z}^{2}} \sum_{\mu=h, v, d} \hat{d}_{j, \mathbf{k}}^{\mu} \Psi_{j, \mathbf{k}}^{\mu}(u ; v),
$$

em que a soma em $j \geq l$ é uma soma finita, pois para a estimação dos coeficientes é utilizada uma quantidade finita de observações, e consequentemente, como a soma em $\mathbf{k} \in \mathbb{Z}^{2}$ depende de $l$ e $j$, também é uma soma finita.

Supondo que

$$
C(u ; v)=\int_{0}^{v} \int_{0}^{u} c(r ; s) d r d s
$$

voltando a (3.1), podemos escrever

$$
\begin{aligned}
c_{l, \mathbf{k}} & =\int_{[0,1]^{2}} C(u ; v) \Phi_{l, \mathbf{k}}(u ; v) d u d v=\int_{[0,1]^{2}}\left[\int_{0}^{v} \int_{0}^{u} c(r ; s) d r d s\right] \Phi_{l, \mathbf{k}}(u ; v) d u d v \\
& =\int_{[0,1]^{2}}\left[\int_{s}^{1} \int_{r}^{1} \Phi_{l, \mathbf{k}}(u ; v) d u d v\right] c(r ; s) d r d s,
\end{aligned}
$$

onde $c(r ; s)$ é a densidade cópula.

Fazendo $r=F(x)$ e $s=G(y)$, como feito em Genest et al. (2009), e sabendo que

$$
h(x ; y)=\frac{\partial^{2}}{\partial x \partial y} H(x ; y),
$$


em que

$$
\begin{aligned}
\frac{\partial^{2}}{\partial x \partial y} H(x ; y) & =\frac{\partial^{2}}{\partial F(x) \partial G(y)} C(F(x) ; G(y)) \frac{\partial}{\partial x} F(x) \frac{\partial}{\partial y} G(y) \\
& =c(F(x) ; G(y)) \cdot f(x) \cdot g(y)
\end{aligned}
$$

sendo $f($.$) e g($.$) as densidades marginais de X$ e $Y$, tem-se que

$$
\begin{aligned}
c_{l, \mathbf{k}} & =\int_{\mathbb{R}^{2}}\left[\int_{G(y)}^{1} \int_{F(x)}^{1} \Phi_{l, \mathbf{k}}(u ; v) d u d v\right] h(x ; y) d x d y \\
& =\mathbb{E}_{h(x, y)}\left[\int_{G(Y)}^{1} \int_{F(X)}^{1} \Phi_{l, \mathbf{k}}(u ; v) d u d v\right] .
\end{aligned}
$$

Muitas vezes, são propostos estimadores que utilizam somente a função escala na expansão da função a ser estimada. A utilização somente da função escala vem de suposições dadas no seguinte teorema:

Teorema 3.1: Suponha que $\phi$ seja uma função contínua com suporte compacto satisfazendo a condição de ortonormalidade: $\int \phi(x-k) \phi(x-l) d x=\delta_{k l}$. Seja $V_{j}$ o espaço gerado por $\left\{\phi\left(2^{j}-k\right) ; k \in \mathbb{Z}\right\}$. Então as seguintes condições são satisfeitas:

- O espaço $V_{j}$ satisfaz a condição $\cap V_{j}=\{0\}$

- Se as seguintes condições adicionais são satisfeitas por $\phi$,

1. $\int \phi(x) d x=1$ (Normalização)

2. $\phi(x)=\sum_{k} p_{k} \phi(2 x-k)$, para algum número finito de constantes $p_{k}$,

então o subespaço $V_{j}$ associado é denso, ou seja, qualquer função pertencente a $L^{2}(\mathbb{R})$ pode ser aproximada por funções em $V_{j}$, para um $j$ grande o suficiente.

Em particular, se a função $\phi$ é contínua, com suporte compacto e satisfaz as condições 1 e 2 do Teorema 3.1, além da ortonormalidade, então a coleção de espaços $\left\{V_{j}, j \in \mathbb{Z}\right\}$ forma uma análise de multiresolução.

Demonstração: Ver teorema 5.17 em Boggess and Narcowich (2001). 
Assim, no cenário das cópulas, vamos supor que:

$$
C_{l}(u ; v)=\sum_{\mathbf{k}} c_{l, \mathbf{k}} \Phi_{l, \mathbf{k}}(u ; v),
$$

e

$$
\hat{C}_{l}(u ; v)=\sum_{\mathbf{k}} \hat{c}_{l, \mathbf{k}} \Phi_{l, \mathbf{k}}(u ; v),
$$

em que $C_{l}(u ; v)$ indica e expansão da Cópula utilizando somente a função escala.

\subsection{Estimação para o caso iid}

Considerando $\left(X_{1} ; Y_{1}\right), \ldots,\left(X_{n} ; Y_{n}\right)$ uma amostra aleatória de uma função distribuição conjunta $H(. ;$.$) , e F_{n}$ e $G_{n}$ funções de distribuição empírica associadas a $F($.$) e$ $G\left(\right.$.)(funções de distribuição marginal de $X$ e $Y$ ), o estimador proposto para $c_{l, \mathbf{k}}$, de acordo com (3.3), é dado por:

$$
\hat{c}_{l, \mathbf{k}}=\frac{1}{n} \sum_{i=1}^{n}\left[\int_{G\left(Y_{i}\right)}^{1} \int_{F\left(X_{i}\right)}^{1} \Phi_{l, \mathbf{k}}(u ; v) d u d v\right],
$$

que é motivado por (3.3).

Porém, note que $F\left(X_{i}\right)$ e $G\left(Y_{i}\right)$ não são conhecidas. Então, um outro estimador é proposto, substituindo as funções distribuição por suas respectivas funções de distribuições empíricas. Logo, tem-se o estimador:

$$
\tilde{c}_{l, \mathbf{k}}=\frac{1}{n} \sum_{i=1}^{n}\left[\int_{G_{n}\left(Y_{i}\right)}^{1} \int_{F_{n}\left(X_{i}\right)}^{1} \Phi_{l, \mathbf{k}}(u ; v) d u d v\right],
$$

em que $F_{n}\left(X_{i}\right)=\frac{1}{n} \sum_{k=1}^{n} \mathbb{I}\left\{X_{k} \leq X_{i}\right\}$ e $G_{n}\left(Y_{i}\right)=\frac{1}{n} \sum_{k=1}^{n} \mathbb{I}\left\{Y_{k} \leq Y_{i}\right\}$.

Para investigar e estudar o comportamento do estimador $\tilde{c}_{l, \mathbf{k}}$, será considerado o risco quadrático, denominado erro quadratico médio integrado (MISE), definido por:

$$
\operatorname{MISE}\left(\tilde{C}_{l}(u ; v), C(u ; v)\right)=\mathbb{E}_{h(x, y)}\left\|\tilde{C}_{l}(u ; v)-C(u ; v)\right\|_{2}^{2} .
$$

Sejam as suposições: 
(S1) $C$ pertençe a $L_{2}\left([0,1]^{2}\right)$, e também pertençe a uma bola de raio $M>0$ em um espaço de besov $\mathfrak{B}_{2}^{s, q}$;

(S2) $\phi$ satisfaz as condições do Teorema 3.1.

Considerando o estimador dado em (3.6) e a função de perda dada em (3.7), tem-se o seguinte resultado:

Teorema 3.2: Suponha que (S1) e (S2) sejam válidas. Dada uma amostra de uma função distribuição conjunta $H(. ;$.$) de tamanho n$, com função cópula $C(. ;$. desconhecida, escolha $l^{*} \in \mathbb{N}$ tal que

$$
2^{l^{*}} \leq n^{\frac{1}{2(s+4)}}<2^{l^{*}+1}
$$

Seja $\tilde{C}_{l^{*}}(. ;$.$) um estimador de C(. ;$.$) , considerando o nível l^{*}$. Então existe uma constante $K>0$, tal que

$$
\sup _{C \in \mathfrak{B}_{s, 2}^{q}(M)} n^{\frac{s+2}{s+4}} \operatorname{MISE}\left(\tilde{C}_{l^{*}}(u ; v), C(u ; v)\right) \leq K .
$$

Sendo assim, $r_{n}=n^{\frac{-(s+2)}{s+4}}$ é conhecida como a taxa de convergência alcançada no espaço de Besov pelo procedimento linear, a partir do estimador proposto.

Note que a escolha do nível $l^{*}$ ótimo para a estimação da cópula utilizando somente a função escala, depende da informação prévia sobre o grau de regularidade $s$ da cópula a ser estimada, que é desconhecida, indicando que este procedimento não é direcionado pelos dados. Sendo assim, uma forma de escolher o nível mais adequado, é partir do maior possível nível $J$ a ser adotado.

Neste trabalho, $J$ assume valor tal que

$$
2^{J} \leq \sqrt{n} \leq 2^{J+1}
$$

em que $l^{*}=J, J-1, J-2, \ldots, 1$. A partir disso, são analisadas as estimativas das cópulas em vários níveis; $l^{*}$ ótimo é o que apresenta melhores resultados em termos de Viés e Erro Quadrático Médio(EQM). 


\section{Demonstração do Teorema 3.2}

Substituindo (3.1) e (3.4) em (3.7), e considerando uma base ortonormal para a expansão, tem-se que

$$
\begin{aligned}
\mathbb{E}_{h(x, y)}\left\|\tilde{C}_{l}(u ; v)-C(u ; v)\right\|_{2}^{2} & \leq \mathbb{E}_{h(x, y)}\left\|\tilde{C}_{l}(u ; v)-C_{l}(u ; v)\right\|_{2}^{2} \\
& +\left\|\sum_{j \geq l} \sum_{\mathbf{k} \in \mathbb{Z}^{2}} \sum_{\mu=h, v, d} d_{j, \mathbf{k}}^{\mu} \Psi_{j, \mathbf{k}}^{\mu}(u ; v)\right\|_{2}^{2} .
\end{aligned}
$$

Logo a equação (3.8) é dividida em duas partes. Analisando separadamente, tem-se que:

$2^{\mathrm{a}}$ parte de (3.8):

Se $C$ pertence ao espaço de Besov $\mathfrak{B}_{p}^{s, q}$, com $s>0,1 \leq p, q<\infty$, então

$$
\|C\|_{s, p, q}=\left\|c_{l .}\right\|_{p}+\left(\sum_{j \geq l}\left(2^{j\left(s+\frac{2}{p}+1\right)}\left\|d_{j .}\right\|_{p}\right)^{q}\right)^{\frac{1}{q}},
$$

onde $\left\|c_{l}\right\|$ é a norma da sequência $\left\{c_{l, \mathbf{k}}, \mathbf{k} \in \mathbb{Z}^{2}\right\}$ para $l$ fixado, assim como $\left\|d_{j}.\right\|$ é norma da sequência $\left\{d_{j, \mathbf{k}}^{\mu}, j \geq l, \mathbf{k} \in \mathbb{Z}^{2}, \mu=h, v, d\right\}$ para $j$ fixado.

Sob a suposição (S1), tem-se que

$$
\begin{aligned}
& \left\|\sum_{j \geq l} \sum_{\mathbf{k}, \mu} d_{j, \mathbf{k}}^{\mu} \Psi_{j, \mathbf{k}}^{\mu}(u ; v)\right\|_{2} \leq \sum_{j \geq l} 2^{j(s+2)}\left\|\sum_{\mathbf{k}, \mu} d_{j, \mathbf{k}}^{\mu} \Psi_{j, \mathbf{k}}^{\mu}(u ; v)\right\|_{2} 2^{-j(s+2)} \\
\leq & \left(\sum_{j \geq l}\left(2^{j(s+2)}\left\|\sum_{\mathbf{k}, \mu} d_{j, \mathbf{k}}^{\mu} \Psi_{j, \mathbf{k}}^{\mu}(u ; v)\right\|_{2}\right)^{q}\right)^{1 / q}\left(\sum_{j \geq l}\left(2^{-j(s+2)}\right)^{q^{\prime}}\right)^{1 / q^{\prime}},
\end{aligned}
$$

pela desigualdade de Hölder, $\operatorname{com} \frac{1}{q}+\frac{1}{q^{\prime}}=1$.

Logo,

$$
\begin{aligned}
& \left\|\sum_{j \geq l} \sum_{\mathbf{k}, \mu} d_{j, \mathbf{k}}^{\mu} \Psi_{j, \mathbf{k}}^{\mu}(u ; v)\right\|_{2} \leq\|C\|_{s, 2, q}\left(\sum_{j \geq l} 2^{-j(s+2) q^{\prime}}\right)^{1 / q^{\prime}} \Rightarrow \\
& \left\|\sum_{j \geq l} \sum_{\mathbf{k}, \mu} d_{j, \mathbf{k}}^{\mu} \Psi_{j, \mathbf{k}}^{\mu}(u ; v)\right\|_{2}^{2} \leq M 2^{-2 l(s+2)},
\end{aligned}
$$


o que conclui a segunda parte de (3.8).

\section{$1^{\text {a }}$ parte de (3.8):}

Estudar o comportamento de $\mathbb{E}_{h(x, y)}\left\|\tilde{C}_{l}(u ; v)-C_{l}(u ; v)\right\|_{2}^{2}$ seria complicado, pelo fato de utilizar estimadores empíricos para $F\left(X_{i}\right)$ e $G\left(Y_{i}\right)$.

Sendo assim, como proposto em Genest et al. (2009), faz-se:

$$
\begin{array}{r}
\mathbb{E}_{h(x, y)}\left\|\tilde{C}_{l}(u ; v)-C_{l}(u ; v)\right\|_{2}^{2}=\mathbb{E}_{h(x, y)}\left\|\tilde{C}_{l}(u ; v)-C_{l}(u ; v)+\hat{C}_{l}(u ; v)-\hat{C}_{l}(u ; v)\right\|_{2}^{2} \\
\leq 2 \mathbb{E}_{h(x, y)}\left\|\tilde{C}_{l}(u ; v)-\hat{C}_{l}(u ; v)\right\|_{2}^{2}+2 \mathbb{E}_{h(x, y)}\left\|\hat{C}_{l}(u ; v)-C_{l}(u ; v)\right\|_{2}^{2},
\end{array}
$$

que, na verdade, se resume em analisar o risco quadrático entre o estimador proposto e o auxiliar, dado por (3.5) e (3.6), e entre o auxiliar e a verdadeira cópula, o que algébricamente se torna mais viável que estudar o erro quadrático entre o estimador proposto e a verdadeira cópula, definida em (3.4).

Desenvolvendo (3.9) separadamente, sob a suposição (S1) tem-se que:

$1^{\mathrm{a}}$ parte de (3.9):

$$
\begin{aligned}
\mathbb{E}_{h(x, y)}\left\|\tilde{C}_{l}(u ; v)-\hat{C}_{l}(u ; v)\right\|_{2}^{2} & =\mathbb{E}_{h(x, y)}\left[\int_{0}^{1} \int_{0}^{1}\left(\tilde{C}_{l}(u ; v)-\hat{C}_{l}(u ; v)\right)^{2} d u d v\right] \\
& =\mathbb{E}_{h(x, y)}\left[\int_{[0,1]^{2}}\left(\sum_{\mathbf{k}} \tilde{c}_{l, \mathbf{k}} \Phi_{l, \mathbf{k}}(u ; v)-\sum_{\mathbf{k}} \hat{c}_{l, \mathbf{k}} \Phi_{l, \mathbf{k}}(u ; v)\right)^{2} d u d v\right] \\
& =\mathbb{E}_{h(x, y)}\left[\int_{[0,1]^{2}}\left(\sum_{\mathbf{k}}\left(\tilde{c}_{l, \mathbf{k}}-\hat{c}_{l, \mathbf{k}}\right) \Phi_{l, \mathbf{k}}(u ; v)\right)^{2} d u d v\right] \\
& =\sum_{\mathbf{k}} \mathbb{E}_{h(x, y)}\left[\left(\tilde{c}_{l, \mathbf{k}}-\hat{c}_{l, \mathbf{k}}\right)^{2}\right] .
\end{aligned}
$$

Substituindo os estimadores, tem-se: 


$$
\begin{aligned}
{\left[\left(\tilde{c}_{l, \mathbf{k}}-\hat{c}_{l, \mathbf{k}}\right)^{2}\right] } & =\left(\frac{1}{n} \sum_{i=1}^{n} \int_{G_{n}\left(Y_{i}\right)}^{1} \int_{F_{n}\left(X_{i}\right)}^{1} \Phi_{l, \mathbf{k}}(u ; v) d u d v-\frac{1}{n} \sum_{i=1}^{n} \int_{G\left(Y_{i}\right)}^{1} \int_{F\left(X_{i}\right)}^{1} \Phi_{l, \mathbf{k}}(u ; v) d u d v\right)^{2} \\
\leq & \left(\left\|\Phi_{l, \mathbf{k}}(u ; v)\right\|_{\infty}\right)^{2}\left\{\frac { 1 } { n } \sum _ { i = 1 } ^ { n } \left[\left(1-G_{n}\left(Y_{i}\right)\right)\left(1-F_{n}\left(X_{i}\right)\right)\right.\right. \\
& -\left(1-G\left(Y_{i}\right)\right)\left(1-F\left(X_{i}\right)\right)+\left(1-F_{n}\left(X_{i}\right)\right)\left(1-G\left(Y_{i}\right)\right) \\
& \left.\left.-\left(1-F_{n}\left(X_{i}\right)\right)\left(1-G\left(Y_{i}\right)\right)\right]\right\}^{2} \\
& =\left(\left\|\Phi_{l, \mathbf{k}}(u ; v)\right\|_{\infty}\right)^{2}\left\{\frac { 1 } { n } \sum _ { i = 1 } ^ { n } \left[\left(1-F_{n}\left(X_{i}\right)\right)\left(G\left(Y_{i}\right)-G_{n}\left(Y_{i}\right)\right)\right.\right. \\
& \left.\left.+\left(1-G\left(Y_{i}\right)\right)\left(F\left(X_{i}\right)-F_{n}\left(X_{i}\right)\right)\right]\right\}^{2} \\
\leq & \left(\left\|\Phi_{l, \mathbf{k}}(u ; v)\right\|_{\infty}\right)^{2}\left\{\frac{1}{n} \sum_{i=1}^{n}\left[\left(G\left(Y_{i}\right)-G_{n}\left(Y_{i}\right)\right)+\left(F\left(X_{i}\right)-F_{n}\left(X_{i}\right)\right)\right]\right\}^{2} \\
\leq & \left(\left\|\Phi_{l, \mathbf{k}}(u ; v)\right\|_{\infty}\right)^{2}\left\{\frac{1}{n} \sum_{i=1}^{n}\left[\left|G\left(Y_{i}\right)-G_{n}\left(Y_{i}\right)\right|+\left|F\left(X_{i}\right)-F_{n}\left(X_{i}\right)\right|\right]\right\}^{2}
\end{aligned}
$$

Defina:

$$
F\left(X_{i}\right)-F_{n}\left(X_{i}\right)=\Delta\left(X_{i}\right) \text { e } G\left(Y_{i}\right)-G_{n}\left(Y_{i}\right)=\Delta\left(Y_{i}\right),
$$

em que $\Delta\left(Y_{i}\right)=\Delta\left(Y_{i}\right)\left(\mathbb{I}\left\{\left|\Delta\left(Y_{i}\right)\right|>\epsilon\right\}+\mathbb{I}\left\{\left|\Delta\left(Y_{i}\right)\right| \leq \epsilon\right\}\right)$, para $\epsilon>0$ fixado.

Sendo assim,

$$
\left[\left(\tilde{c}_{l, \mathbf{k}}-\hat{c}_{l, \mathbf{k}}\right)^{2}\right]=\left(\left\|\Phi_{l, \mathbf{k}}(u ; v)\right\|_{\infty}\right)^{2}\left\{\frac{1}{n} \sum_{i=1}^{n}\left[\left|\Delta\left(X_{i}\right)\right|+\left|\Delta\left(Y_{i}\right)\right|\right]\right\}^{2} .
$$

Logo,

$$
\sum_{i=1}^{n}\left|\Delta\left(X_{i}\right)\right| \leq n \epsilon+n\left(\mathbb{I}\left\{\left|\Delta\left(X_{i}\right)\right|>\epsilon\right\}\right)
$$


De maneira análoga, é feito para $\sum_{i=1}^{n}\left|\Delta\left(Y_{i}\right)\right|$.

Assim,

$$
\begin{aligned}
{\left[\left(\tilde{c}_{l, \mathbf{k}}-\hat{c}_{l, \mathbf{k}}\right)^{2}\right] } & \leq\left(\left\|\Phi_{l, \mathbf{k}}(u ; v)\right\|_{\infty}\right)^{2}\left\{\mathbb{I}\left\{\left|\Delta\left(X_{i}\right)\right|>\epsilon\right\}+2 \epsilon+\mathbb{I}\left\{\left|\Delta\left(Y_{i}\right)\right|>\epsilon\right\}\right\}^{2} \\
& =\left(\left\|\Phi_{l, \mathbf{k}}(u ; v)\right\|_{\infty}\right)^{2}\left\{\mathbb{I}^{2}\left\{\left|\Delta\left(X_{i}\right)\right|>\epsilon\right\}+4 \epsilon^{2}+\mathbb{I}^{2}\left\{\left|\Delta\left(Y_{i}\right)\right|>\epsilon\right\}\right. \\
& +4 \epsilon \mathbb{I}\left\{\left|\Delta\left(X_{i}\right)\right|>\epsilon\right\}+4 \epsilon \mathbb{I}\left\{\left|\Delta\left(Y_{i}\right)\right|>\epsilon\right\} \\
& \left.+2\left(\mathbb{I}\left\{\left|\Delta\left(X_{i}\right)\right|>\epsilon\right\} \mathbb{I}\left\{\left|\Delta\left(Y_{i}\right)\right|>\epsilon\right\}\right)\right\} .
\end{aligned}
$$

Mas, pela definição de função indicadora, tem-se que

$$
\mathbb{I}\left\{\left|\Delta\left(X_{i}\right)\right|>\epsilon\right\} \mathbb{I}\left\{\left|\Delta\left(Y_{i}\right)\right|>\epsilon\right\} \leq \mathbb{I}\left\{\left|\Delta\left(X_{i}\right)\right|>\epsilon\right\}+\mathbb{I}\left\{\left|\Delta\left(Y_{i}\right)\right|>\epsilon\right\},
$$

$\log 0$

$$
\begin{aligned}
{\left[\left(\tilde{c}_{l, \mathbf{k}}-\hat{c}_{l, \mathbf{k}}\right)^{2}\right] } & \leq\left(\left\|\Phi_{l, \mathbf{k}}(u ; v)\right\|_{\infty}\right)^{2}\left\{3 \mathbb{I}\left\{\left|\Delta\left(X_{i}\right)\right|>\epsilon\right\}+4 \epsilon \mathbb{I}\left\{\left|\Delta\left(X_{i}\right)\right|>\epsilon\right\}+4 \epsilon^{2}\right. \\
& \left.+3 \mathbb{I}\left\{\left|\Delta\left(Y_{i}\right)\right|>\epsilon\right\}+4 \epsilon \mathbb{I}\left\{\left|\Delta\left(Y_{i}\right)\right|>\epsilon\right\}\right\} \\
& =\left(\left\|\Phi_{l, \mathbf{k}}(u ; v)\right\|_{\infty}\right)^{2}\left\{4 \epsilon^{2}+(3+4 \epsilon)\left[\mathbb{I}\left\{\left|\Delta\left(X_{i}\right)\right|>\epsilon\right\}+\mathbb{I}\left\{\left|\Delta\left(Y_{i}\right)\right|>\epsilon\right\}\right]\right\}
\end{aligned}
$$

Logo, voltando a (3.10),

$$
\begin{aligned}
\sum_{\mathbf{k}} \mathbb{E}_{h(x, y)}\left[\left(\tilde{c}_{l, \mathbf{k}}-\hat{c}_{l, \mathbf{k}}\right)^{2}\right] & \leq \sum_{\mathbf{k}}\left(\left\|\Phi_{l, \mathbf{k}}(u ; v)\right\|_{\infty}\right)^{2} \mathbb{E}_{h(x, y)}\left\{4 \epsilon^{2}\right. \\
& \left.+(3+4 \epsilon)\left[\mathbb{I}\left\{\left|\Delta\left(X_{i}\right)\right|>\epsilon\right\}+\mathbb{I}\left\{\left|\Delta\left(Y_{i}\right)\right|>\epsilon\right\}\right]\right\}
\end{aligned}
$$

Mas,

$$
\begin{aligned}
\left\|\Phi_{l, \mathbf{k}}(u ; v)\right\|_{\infty} & =\left\|2^{l} \Phi\left(2^{l} u-k_{1} ; 2^{l} v-k_{2}\right)\right\|_{\infty}=2^{l} \sup _{\left(2^{l} u-k_{1} ; 2^{l} v-k_{2}\right)}\left|\Phi\left(2^{l} u-k_{1} ; 2^{l} v-k_{2}\right)\right| \\
& =2^{l}\|\Phi(w ; z)\|_{\infty}, \text { em que } w=2^{l} u-k_{1} \text { e } z=2^{l} v-k_{2},
\end{aligned}
$$


com $k_{1}=0, \ldots, 2^{l}-1, k_{2}=0, . ., 2^{l}-1$.

Portanto,

$$
\begin{aligned}
\sum_{\mathbf{k}} \mathbb{E}_{h(x, y)}\left[\left(\tilde{c}_{l, \mathbf{k}}-\hat{c}_{l, \mathbf{k}}\right)^{2}\right] & \leq 2^{2 l}\left(\|\Phi(w ; z)\|_{\infty}\right)^{2} \sum_{\mathbf{k}}\left\{4 \epsilon^{2}\right. \\
& \left.+(3+4 \epsilon)\left[P\left\{\left|\Delta\left(X_{i}\right)\right|>\epsilon\right\}+P\left\{\left|\Delta\left(Y_{i}\right)\right|>\epsilon\right\}\right]\right\} .
\end{aligned}
$$

Como $\sum_{\mathrm{k}}=2^{2 l}$, pela desigualdade de Dvoretzky-Kiefer-Wolfowitz ${ }^{1}$, para $\epsilon=$ $\left\{\frac{\delta \log (n)}{2 n}\right\}^{\frac{1}{2}}$, e para $\delta$ suficientemente grande, tem-se

$$
\mathbb{E}_{h(x, y)}\left\|\tilde{C}_{l}(u ; v)-\hat{C}_{l}(u ; v)\right\|_{2}^{2} \leq 2^{4 l}\left\{k_{1} \frac{\log (n)}{n}+k_{2} n^{-\delta}+k_{3}(\log (n))^{\frac{1}{2}} n^{-\left(\delta+\frac{1}{2}\right)}\right\},
$$

com $k_{1}, k_{2}$ e $k_{3}$ dependendo de $\|\Phi(w ; z)\|_{\infty}$ e de $\delta$, concluindo a primeira parte de (3.9).

\section{$2^{\mathrm{a}}$ parte de (3.9):}

Note que

$$
\begin{aligned}
\mathbb{E}_{h(x, y)}\left(\hat{C}_{l}(u ; v)\right) & =\mathbb{E}_{h(x, y)}\left(\sum_{\mathbf{k}} \hat{c}_{l, \mathbf{k}} \Phi_{l, \mathbf{k}}(u ; v)\right)=\sum_{\mathbf{k}} \mathbb{E}_{h(x, y)}\left(\hat{c}_{l, \mathbf{k}}\right) \Phi_{l, \mathbf{k}}(u ; v) \\
& =\sum_{\mathbf{k}} \mathbb{E}_{h(x, y)}\left(\frac{1}{n} \sum_{i=1}^{n}\left[\int_{G\left(Y_{i}\right)}^{1} \int_{F\left(X_{i}\right)}^{1} \Phi_{l, \mathbf{k}}(u ; v) d u d v\right]\right) \Phi_{l, \mathbf{k}}(u ; v) \\
& =\sum_{\mathbf{k}} \frac{1}{n} \sum_{i=1}^{n} \mathbb{E}_{h\left(x_{i}, y_{i}\right)}\left[\int_{G\left(Y_{i}\right)}^{1} \int_{F\left(X_{i}\right)}^{1} \Phi_{l, \mathbf{k}}(u ; v) d u d v\right] \Phi_{l, \mathbf{k}}(u ; v) \\
& =\sum_{\mathbf{k}} c_{l, \mathbf{k}} \Phi_{l, \mathbf{k}}(u ; v)=C_{l}(u ; v),
\end{aligned}
$$

mostrando que $\hat{C}_{l}(u ; v)$ é um estimador não viesado para $C_{l}(u ; v)$.

\footnotetext{
${ }^{1}$ Ver Apêndice A
} 
Sendo assim,

$$
\begin{aligned}
\mathbb{E}_{h(x, y)}\left\|\hat{C}_{l}(u ; v)-C_{l}(u ; v)\right\|_{2}^{2} & =\mathbb{E}_{h(x, y)}\left\|\hat{C}_{l}(u ; v)-\mathbb{E}_{h(x, y)}\left(\hat{C}_{l}(u ; v)\right)\right\|_{2}^{2} \\
& =\mathbb{E}_{h(x, y)}\left[\int_{[0,1]^{2}}\left(\sum_{\mathbf{k}}\left(\hat{c}_{l, \mathbf{k}}-\mathbb{E}_{h(x, y)}\left(\hat{c}_{l, \mathbf{k}}\right)\right) \Phi_{l, \mathbf{k}}(u ; v)\right)^{2} d u d v\right] \\
& =\sum_{\mathbf{k}} \mathbb{E}_{h(x, y)}\left[\left(\hat{c}_{l, \mathbf{k}}-\mathbb{E}_{h(x, y)}\left(\hat{c}_{l, \mathbf{k}}\right)\right)^{2}\right] \\
& =\sum_{\mathbf{k}} \mathbb{E}_{h(x, y)}\left[\left(\frac{1}{n} \sum_{i=1}^{n} \int_{G\left(Y_{i}\right)}^{1} \int_{F\left(X_{i}\right)}^{1} \Phi_{l, \mathbf{k}}(u ; v) d u d v\right.\right. \\
& \left.\left.-\frac{1}{n} \sum_{i=1}^{n} E_{h\left(x_{i}, y_{i}\right)}\left(\int_{G\left(Y_{i}\right)}^{1} \int_{F\left(X_{i}\right)}^{1} \Phi_{l, \mathbf{k}}(u ; v) d u d v\right)\right)^{2}\right] .
\end{aligned}
$$

Seja

$$
\begin{aligned}
W_{i} & =\int_{G\left(Y_{i}\right)}^{1} \int_{F\left(X_{i}\right)}^{1} \Phi_{l, \mathbf{k}}(u ; v) d u d v-\mathbb{E}_{h\left(x_{i}, y_{i}\right)}\left(\int_{G\left(Y_{i}\right)}^{1} \int_{F\left(X_{i}\right)}^{1} \Phi_{l, \mathbf{k}}(u ; v) d u d v\right) \\
& =\int_{G\left(Y_{i}\right)}^{1} \int_{F\left(X_{i}\right)}^{1} \Phi_{l, \mathbf{k}}(u ; v) d u d v-c_{l, \mathbf{k}},
\end{aligned}
$$

então

$$
\mathbb{E}_{h(x, y)}\left\|\hat{C}(u ; v)-\mathbb{E}_{h(x, y)}(\hat{C}(u ; v))\right\|_{2}^{2}=\sum_{\mathbf{k}} \mathbb{E}_{h(x, y)}\left[\left(\frac{1}{n} \sum_{i=1}^{n} W_{i}\right)^{2}\right] .
$$

Note que $W_{i}$ são variáveis aleatórias iid, com $\mathbb{E}_{h\left(x_{i}, y_{i}\right)}\left(W_{i}\right)=0$ e também que

$$
\begin{aligned}
\mathbb{E}_{h(x, y)}\left[\left(W_{i}\right)^{2}\right] & \leq \mathbb{E}_{h(x, y)}\left[\left(\int_{G\left(Y_{i}\right)}^{1} \int_{F\left(X_{i}\right)}^{1} \Phi_{l, \mathbf{k}}(u ; v) d u d v\right)^{2}\right] \\
& =\mathbb{E}_{h(x, y)}\left[\left(\int_{0}^{1} \int_{0}^{1} \Phi_{l, \mathbf{k}}(u ; v) \mathbb{I}\left(F\left(X_{i}\right)<u<1\right) \mathbb{I}\left(G\left(Y_{i}\right)<v<1\right) d u d v\right)^{2}\right] \\
& =\mathbb{E}_{h(x, y)}\left[\left(2 ^ { - l } \int _ { 0 } ^ { 1 } \int _ { 0 } ^ { 1 } \Phi ( w ; z ) \mathbb { I } \left(2^{l} F\left(X_{i}\right)-k_{1}<w<2^{l}-k_{2}\right.\right.\right. \\
& \left.\left.\times \mathbb{I}\left(2^{l} G\left(Y_{i}\right)-k_{2}<z<2^{l}-k_{2}\right) d w d z\right)^{2}\right]<2^{2 l}\|\Phi(w ; z)\|_{\infty}<\infty .
\end{aligned}
$$


Logo, pela desigualdade de Rosenthal ${ }^{2}$, existe $K>0$ tal que

$$
\begin{aligned}
\sum_{\mathbf{k}} \mathbb{E}_{h(x, y)}\left[\left(\frac{1}{n} \sum_{i=1}^{n} W_{i}\right)^{2}\right] & \leq \sum_{\mathbf{k}} \frac{1}{n^{2}} \mathbb{E}_{h(x, y)}\left[\left(\sum_{i=1}^{n} W_{i}\right)^{2}\right] \\
& \leq \sum_{\mathbf{k}} K\left[\sum_{i=1}^{n} \mathbb{E}_{h(x, y)}\left(W_{i}^{2}\right)+\left(\sum_{i=1}^{n} \mathbb{E}_{h(x, y)}\left(W_{i}^{2}\right)\right)\right] \\
& \leq \sum_{\mathbf{k}} \frac{1}{n^{2}} K\left[n 2^{2 l}+\left(n 2^{2 l}\right)\right] \leq \sum_{\mathbf{k}} \frac{1}{n} K^{\prime} 2^{2 l}=K^{\prime} \frac{2^{4 l}}{n} .
\end{aligned}
$$

Sendo assim,

$$
\mathbb{E}_{h(x, y)}\left\|\hat{C}_{l}(u ; v)-C_{l}(u ; v)\right\|_{2}^{2} \leq K^{\prime} \frac{2^{4 l}}{n}
$$

concluindo a segunda parte de (3.9), e consequentemente a primira parte de (3.8).

Combinando os resultados de (3.8) e (3.9), tem-se

$$
\begin{aligned}
\mathbb{E}_{h(x, y)}\left\|\tilde{C}_{l}(u ; v)-C(u ; v)\right\|_{2}^{2} & \leq 2^{4 l+1}\left[k_{1} \frac{\log (n)}{n}+k_{2} n^{-\delta}+k_{3}(\log (n))^{\frac{1}{2}} n^{-\left(\delta+\frac{1}{2}\right)}\right] \\
& +K^{\prime} \frac{2^{4 l+1}}{n}+M 2^{-2 l(s+2)}
\end{aligned}
$$

O termo dado pela primeira parte de (3.9) é conhecido como o erro resultante da aproximação das funções $F\left(X_{i}\right)$ e $G\left(Y_{i}\right)$ pelas suas respectivas funções empíricas, que tende a zero para $n$ tendendo a infinito.

Para calcular a taxa de convergência de (3.11) serão considerados os dois últimos termos, que como podem ser vistos, apresentam comportamentos contrários para diferentes valores de $l$.

Para que a soma

$$
K^{\prime} \frac{2^{4 l+1}}{n}+M 2^{-2 l(s+2)}
$$

\footnotetext{
${ }^{2}$ Ver Apêndice A para mais detalhes
} 
admita um valor mínimo, as duas quantidades devem ser balanceadas. Para isto, é calculado o valor de $l$, em função de $n$ que minimiza (3.11). Mais detalhes sobre esse procedimento podem ser vistos em Härdle et al. (1998).

Sendo assim, para uma amostra de uma função distribuição conjunta $H(. ;$.) de tamanho $n$, com função cópula $C(. ;$.) desconhecida, e supondo que $C(. ;$.$) pertença a$ uma bola de raio $M$ no espaço de Besov $\mathfrak{B}_{2}^{s, q}(M)$, para $s$ fixo, $\mathbb{E}_{h(x, y)}\left\|\tilde{C}_{l}(u ; v)-C(u ; v)\right\|_{2}^{2}$ admite valor mínimo quando $l^{*} \in \mathbb{N}$ assume valor

$$
2^{l^{*}} \leq n^{\frac{2}{s+4}}<2^{l^{*}+1}
$$

Seja $\tilde{C}_{l^{*}}(. ;$.$) o estimador de C(. ;$.$) definido em (3.5). Considerando o nível l^{*}$ dado em (3.12), existe uma constante $K>0$, tal que

$$
\begin{gathered}
\sup _{C \in \mathfrak{B}_{s, 2}^{q}(M)} \operatorname{MISE}\left(\tilde{C}_{l^{*}}(u ; v), C(u ; v)\right) \leq K n^{\frac{-(s+2)}{s+4}} \\
\Rightarrow \sup _{C \in \mathfrak{B}_{s, 2}^{q}(M)} n^{\frac{s+2}{s+4}} \operatorname{MISE}\left(\tilde{C}_{l^{*}}(u ; v), C(u ; v)\right) \leq K,
\end{gathered}
$$

o que garante o resultado. 


\section{Capítulo 4}

\section{Estimação para o caso de séries temporais}

Neste capítulo, utilizando a mesma abordagem que no caso iid e a partir de algumas suposições, será proposto um estimador para a função cópula para o caso de séries temporais.

Seja $\left\{\mathbf{V}_{t}=\left(X_{t} ; Y_{t}\right), t \in \mathbb{Z}\right\}$ um processo estocástico bidimensional estacionário, com função de densidade $h_{t}(x, y)$, para todo $t \in \mathbb{Z}$, e para todo $(x, y) \in \mathbb{R}^{2}$. Sejam $\left\{\mathbf{V}_{t}, t=1, \ldots, T\right\}$ realizações do processo.

Para propor o estimador, são feitas as seguintes suposições:

(S1) Para cada inteiro $h$, existe a função de distribuição conjunta $J\left(\left(X_{t} ; Y_{t}\right) ;\left(X_{t+h} ; Y_{t+h}\right)\right)$ e uma constante positiva $M$, tal que para cada variável aleatória de média zero, limitada $H\left(X_{t} ; Y_{t}\right)$ :

$$
\mathbb{E}\left[\left|H\left(X_{t} ; Y_{t}\right) \cdot H\left(X_{t+h} ; Y_{t+h}\right)\right|\right] \leq M \mathbb{E}\left[\left|H\left(X_{t} ; Y_{t}\right)\right|\right] \mathbb{E}\left[\left|H\left(X_{t+h} ; Y_{t+h}\right)\right|\right]
$$

(S2) O processo estacionário $\left\{\mathbf{V}_{t}, t \in \mathbb{Z}\right\}$ é $\alpha$-mixing ${ }^{3}$, e os coeficientes $\alpha(p)$ são, para

\footnotetext{
${ }^{3}$ Ver Capítulo 2 para mais detalhes
} 
$r>2$ :

$$
\sum_{p=N}^{\infty}[\alpha(p)]^{1-\frac{2}{r}}=O\left(N^{-1}\right)
$$

(S3) $C$ pertence ao espaço de Besov $\mathfrak{B}_{p}^{s, q}$, com $s>0,1 \leq p, q<\infty$.

(S4) $\left\{X_{t}, t \in \mathbb{Z}\right\}$ e $\left\{Y_{t}, t \in \mathbb{Z}\right\}$ são processos $\alpha$-mixing.

Considerando a função de perda

$$
\operatorname{MISE}\left(\tilde{C}_{l}(u ; v), C(u ; v)\right)=\mathbb{E}_{h(x, y)}\left\|\tilde{C}_{l}(u ; v)-C(u ; v)\right\|_{2}^{2}
$$

tem-se o seguinte resultado:

Teorema 4.1: Suponha que sejam válidas (S1) - (S4). Dadas as T realizações do processo $\left\{\mathbf{V}_{t}, t \in \mathbb{Z}\right\}$ e seja $\tilde{C}_{l}(. ;$.$) um estimador de C(u ; v)$. Então, existe uma constante $K>0$ tal que

$$
\operatorname{MISE}\left(\tilde{C}_{l}(u ; v), C(u ; v)\right) \leq K\left[\frac{2}{T}+M 2^{-2 l(s+2)}\right] .
$$

\section{Demonstração do Teorema 4.1}

Analogamente ao caso iid, e sob a suposição (S3), tem-se que

$$
\begin{aligned}
\mathbb{E}_{h(x, y)}\left\|\tilde{C}_{l}(u ; v)-C(u ; v)\right\|_{2}^{2} & \leq \mathbb{E}_{h(x, y)}\left\|\tilde{C}_{l}(u ; v)-C_{l}(u ; v)\right\|_{2}^{2} \\
& +\left\|\sum_{j \geq l} \sum_{\mathbf{k} \in \mathbb{Z}^{2}} \sum_{\mu=h, v, d} d_{j, \mathbf{k}}^{\mu} \Psi_{j, \mathbf{k}}^{\mu}(u ; v)\right\|_{2}^{2}
\end{aligned}
$$

em que

$$
\left\|\sum_{j \geq l} \sum_{\mathbf{k}, \mu} d_{j, \mathbf{k}}^{\mu} \Psi_{j, \mathbf{k}}^{\mu}(u ; v)\right\|_{2}^{2} \leq M 2^{-2 l(s+2)}
$$


A primeira parte de (4.1) é dividida em duas partes

$$
\begin{aligned}
\mathbb{E}_{h(x, y)}\left\|\tilde{C}_{l}(u ; v)-C_{l}(u ; v)\right\|_{2}^{2} & \leq 2 \mathbb{E}_{h(x, y)}\left\|\tilde{C}_{l}(u ; v)-\hat{C}_{l}(u ; v)\right\|_{2}^{2} \\
& +2 \mathbb{E}_{h(x, y)}\left\|\hat{C}_{l}(u ; v)-C_{l}(u ; v)\right\|_{2}^{2},
\end{aligned}
$$

em que, analogamente ao caso iid, é proposto o estimador auxiliar

$$
\tilde{c}_{l, \mathbf{k}}=\frac{1}{T} \sum_{t=1}^{T}\left[\int_{G_{T}\left(Y_{t}\right)}^{1} \int_{F_{T}\left(X_{t}\right)}^{1} \Phi_{l, \mathbf{k}}(u ; v) d u d v\right],
$$

$\operatorname{com} t=\{1, \ldots, T\}$. Logo

$$
\tilde{C}_{l}(u ; v)=\sum_{\mathbf{k}} \tilde{c}_{l, \mathbf{k}} \Phi_{l, \mathbf{k}}(u ; v)
$$

$2^{\mathrm{a}}$ parte de $(4.2)$ :

$$
\begin{aligned}
\mathbb{E}_{h(x, y)}\left\|\hat{C}_{l}(u ; v)-C_{l}(u ; v)\right\|_{2}^{2} & \leq \mathbb{E}_{h(x, y)}\left\|\hat{C}(u ; v)-\mathbb{E}_{h(x, y)}(\hat{C}(u ; v))\right\|_{2}^{2} \\
& +\left\|\mathbb{E}_{h(x, y)}(\hat{C}(u ; v))-C(u ; v)\right\|_{2}^{2}
\end{aligned}
$$

Como pode ser observado, o risco também é analisado em duas partes, uma chamada de erro estocástico e a outra, erro do viés.

Porém, analogamente ao caso iid, e pela estacionariedade, tem-se que

$$
\left\|\mathbb{E}_{h(x, y)}(\hat{C}(u ; v))-C(u ; v)\right\|_{2}^{2}=0 .
$$

Então, desenvolvendo a primeira parte da desigualdade (4.3), tem-se

$$
\begin{aligned}
\mathbb{E}_{h(x, y)}\left\|\hat{C}(u ; v)-\mathbb{E}_{h(x, y)}(\hat{C}(u ; v))\right\|_{2}^{2} & =\sum_{\mathbf{k}} \mathbb{E}_{h(x, y)}\left[\left(\hat{c}_{l, \mathbf{k}}-\mathbb{E}_{h(x, y)}\left(\hat{c}_{l, \mathbf{k}}\right)\right)^{2}\right] \\
& =\sum_{\mathbf{k}} \mathbb{E}_{h(x, y)}\left[\left(\frac{1}{T} \sum_{t=1}^{T} W_{t}\right)^{2}\right]
\end{aligned}
$$


em que

$$
W_{t}=\int_{G\left(Y_{t}\right)}^{1} \int_{F\left(X_{t}\right)}^{1} \Phi_{l, \mathbf{k}}(u ; v) d u d v-\mathbb{E}_{h(x, y)}\left(\int_{G\left(Y_{t}\right)}^{1} \int_{F\left(X_{t}\right)}^{1} \Phi_{l, \mathbf{k}}(u ; v) d u d v\right) .
$$

Pela estacionariedade do processo:

$$
\begin{aligned}
\sum_{\mathbf{k}} \mathbb{E}_{h(x, y)}\left[\left(\frac{1}{T} \sum_{t=1}^{T} W_{t}\right)^{2}\right] & =\frac{1}{T^{2}} \sum_{\mathbf{k}} \mathbb{E}_{h(x, y)}\left[\sum_{t=1}^{T} W_{t}^{2}+\sum_{t=1}^{T} \sum_{\substack{h=1 \\
t \neq h}}^{T} W_{t} W_{h}\right] \\
& =\frac{1}{T^{2}} \sum_{\mathbf{k}}\left[\sum_{t=1}^{T} \mathbb{E}_{h(x, y)}\left(W_{t}^{2}\right)+2 \sum_{h=1}^{T-1}(T-h) \mathbb{E}_{h(x, y)}\left(W_{T} W_{T-h}\right)\right] \\
& =\frac{1}{T^{2}} \sum_{\mathbf{k}}\left[\sum_{t=1}^{T} \mathbb{E}_{h(x, y)}\left(W_{t}^{2}\right)\right] \\
& +\frac{2}{T^{2}} \sum_{\mathbf{k}}\left[\sum_{h=1}^{T-1}(T-h) \mathbb{E}_{h(x, y)}\left(W_{T} W_{T-h}\right)\right]
\end{aligned}
$$

$1^{\mathrm{a}}$ parte de $(4.4)$ :

$$
\begin{aligned}
\frac{1}{T^{2}} \sum_{\mathbf{k}}\left[\sum_{t=1}^{T} \mathbb{E}_{h(x, y)}\left(W_{t}^{2}\right)\right] & \leq \frac{1}{T^{2}} \sum_{\mathbf{k}} \sum_{t=1}^{T} \mathbb{E}_{h(x, y)}\left[\left(\int_{G\left(Y_{t}\right)}^{1} \int_{F\left(X_{t}\right)}^{1} \Phi_{l, \mathbf{k}}(u ; v) d u d v\right)^{2}\right] \\
& =\frac{1}{T^{2}} \sum_{\mathbf{k}} \sum_{t=1}^{T} \mathbb{E}_{h(x, y)}\left[\left(2^{l} \int_{G\left(Y_{t}\right)}^{1} \int_{F\left(X_{t}\right)}^{1} \phi\left(2^{l} u-k_{1}\right) \phi\left(2^{l} v-k_{2}\right) d u d v\right)^{2}\right] .
\end{aligned}
$$

Fazendo $2^{l} u-k_{1}=r$ e $2^{l} v-k_{2}=s$, tem-se

$$
\begin{aligned}
\frac{1}{T^{2}} \sum_{\mathbf{k}}\left[\sum_{t=1}^{T} \mathbb{E}_{h(x, y)}\left(W_{t}^{2}\right)\right] & \leq \frac{1}{T^{2}} \sum_{\mathbf{k}} \sum_{t=1}^{T} \mathbb{E}_{h(x, y)}\left[\left(2^{-l} \int_{2^{l} G\left(Y_{t}\right)-k_{2}}^{2^{l}-k_{2}} \int_{2^{l} F\left(X_{t}\right)-k_{1}}^{2^{l}-k_{1}} \phi(r) \phi(s) d r d s\right)^{2}\right] \\
& \leq \frac{2^{-2 l}}{T^{2}} \sum_{\mathbf{k}}\left[\sum_{t=1}^{T} \mathbb{E}_{h(x, y)}\left(M_{1}^{2}\right)\right]
\end{aligned}
$$

em que

$$
M_{1}=\sup _{l, \mathbf{k}}\left\|\int_{2^{l} G\left(Y_{t}\right)-k_{2}}^{2^{l}-k_{2}} \int_{2^{l} F\left(X_{t}\right)-k_{1}}^{2^{l}-k_{1}} \phi(r) \phi(s) d r d s\right\|_{\infty} .
$$


Assim,

$$
\begin{aligned}
\frac{1}{T^{2}} \sum_{\mathbf{k}}\left[\sum_{t=1}^{T} \mathbb{E}_{h(x, y)}\left(W_{t}^{2}\right)\right] & \leq \frac{2^{-2 l}}{T^{2}} \sum_{\mathbf{k}}\left[\sum_{t=1}^{T} \mathbb{E}_{h(x, y)}\left(M_{1}^{2}\right)\right]=\frac{2^{-2 l}}{T} \sum_{\mathbf{k}} M_{1}^{2} \\
& \leq \frac{M_{1}^{2}}{T}
\end{aligned}
$$

o que conclui a primeira parte de (4.4).

$2^{\mathrm{a}}$ parte de (4.4):

Considere a soma em $h$ dividida em duas partes, a primeira até o instante $\eta$, e a segunda de $(\eta+1)$ até $(T-1)$, em que

$$
\begin{aligned}
\frac{2}{T^{2}} \sum_{\mathbf{k}}\left[\sum_{h=1}^{T-1}(T-h) \mathbb{E}_{h(x, y)}\left(W_{T} W_{T-h}\right)\right] & =\frac{2}{T^{2}} \sum_{\mathbf{k}}\left[\sum_{h=1}^{\eta}(T-h) \mathbb{E}_{h(x, y)}\left(W_{T} W_{T-h}\right)\right. \\
& \left.+\sum_{h=\eta+1}^{T-1}(T-h) \mathbb{E}_{h(x, y)}\left(W_{T} W_{T-h}\right)\right] .
\end{aligned}
$$

$1^{\mathrm{a}}$ parte de (4.5):

$$
\begin{array}{r}
\frac{2}{T^{2}} \sum_{\mathbf{k}}\left[\sum_{h=1}^{\eta}(T-h) \mathbb{E}_{h(x, y)}\left(W_{T} W_{T-h}\right)\right] \leq \frac{2}{T^{2}} \sum_{\mathbf{k}}\left[T \sum_{h=1}^{\eta}\left(1-\frac{h}{T}\right) \mathbb{E}_{h(x, y)}\left|W_{T} W_{T-h}\right|\right] \\
\leq \frac{2 M}{T} \sum_{\mathbf{k}}\left[\sum_{h=1}^{\eta}\left(1-\frac{h}{T}\right)\left(\mathbb{E}_{h(x, y)}\left|W_{T}\right|\right)\left(\mathbb{E}_{h(x, y)}\left|W_{T-h}\right|\right)\right],
\end{array}
$$

em que, utilizou-se a suposição (S1).

Mas, sabe-se que, para todo $t=1, \ldots T$

$$
\begin{aligned}
\mathbb{E}_{h(x, y)}\left(\left|W_{t}\right|\right) & =\mathbb{E}_{h(x, y)}\left|\int_{G\left(Y_{t}\right)}^{1} \int_{F\left(X_{t}\right)}^{1} \Phi_{l, \mathbf{k}}(u ; v) d u d v-\mathbb{E}_{h(x, y)}\left(\int_{G\left(Y_{t}\right)}^{1} \int_{F\left(X_{t}\right)}^{1} \Phi_{l, \mathbf{k}}(u ; v) d u d v\right)\right| \\
& =2^{-l} \mathbb{E}_{h(x, y)} \mid \int_{2^{l} G\left(Y_{t}\right)-k_{2}}^{2^{l}-k_{2}} \int_{2^{l} F\left(X_{t}\right)-k_{1}}^{2^{l}-k_{1}} \phi(r) \phi(s) d r d s \\
& -\mathbb{E}_{h(x, y)}\left(\int_{2^{l} G\left(Y_{t}\right)-k_{2}}^{2^{l}-k_{2}} \int_{2^{l} F\left(X_{t}\right)-k_{1}}^{2^{l}-k_{1}} \phi(r) \phi(s) d r d s\right) \mid,
\end{aligned}
$$


em que

$$
\int_{2^{l} G\left(Y_{t}\right)-k_{2}}^{2^{l}-k_{2}} \int_{2^{l} F\left(X_{t}\right)-k_{1}}^{2^{l}-k_{1}} \phi(r) \phi(s) d r d s \leq \int_{\left[a^{\prime}, a\right]^{2}} \phi(r) \phi(s) d r d s,
$$

em que $\left[a^{\prime}, a\right]^{2}$ é a região na qual $\phi(r) \phi(s) \neq 0$, e pela normalização da função escala, tem-se que:

$$
\left|\int_{\left[a^{\prime}, a\right]^{2}} \phi(r) \phi(s) d r d s-\mathbb{E}_{h(x, y)}\left(\int_{\left[a^{\prime}, a\right]^{2}} \phi(r) \phi(s) d r d s\right)\right| \leq 1,
$$

assim, $\mathbb{E}_{h(x, y)}\left(\left|W_{t}\right|\right) \leq 2^{-l}$ uniformemente em $(x ; y)$, para todo $t=1, \ldots, T$.

Logo

$$
\begin{aligned}
\frac{2 M}{T} \sum_{\mathbf{k}}\left[\sum_{h=1}^{\eta}\left(1-\frac{h}{T}\right)\left(\mathbb{E}_{h(x, y)}\left|W_{T}\right|\right)\left(\mathbb{E}_{h(x, y)}\left|W_{T-h}\right|\right)\right] & \leq \frac{2 M}{T} \sum_{\mathbf{k}}\left[\sum_{h=1}^{\eta}\left(1-\frac{h}{T}\right) 2^{-2 l}\right] \\
& \leq \frac{2 M}{T} \sum_{h=1}^{\eta}\left(1-\frac{h}{T}\right) \\
& =\frac{2 M \eta}{T}-\frac{2 M}{T^{2}} \sum_{h=1}^{\eta} h,
\end{aligned}
$$

pois $\sum_{h=1}^{\eta} h$ é finita, cncluindo a primeira parte de (4.5).

$2^{\underline{a}}$ parte de $(4.5)$ :

$$
\begin{aligned}
\frac{2}{T^{2}} \sum_{\mathbf{k}}\left[\sum_{h=\eta+1}^{T-1}(T-h) \mathbb{E}_{h(x, y)}\left(W_{T} W_{T-h}\right)\right] & \leq \frac{2}{T^{2}} \sum_{\mathbf{k}}\left[\sum_{h=\eta+1}^{T-1}(T-h) \mathbb{E}_{h(x, y)}\left(W_{T} W_{T-h}\right) \mid\right. \\
& \leq \frac{2}{T} \sum_{\mathbf{k}}\left[\sum_{h=\eta+1}^{T-1}\left(1-\frac{h}{T}\right)\left|\mathbb{E}_{h(x, y)}\left(W_{T} W_{T-h}\right)\right|\right] \\
& \leq \frac{2}{T} \sum_{\mathbf{k}}\left[\sum_{h=\eta+1}^{T-1}\left|\mathbb{E}_{h(x, y)}\left(W_{T} W_{T-h}\right)\right|\right],
\end{aligned}
$$

$\operatorname{mas}\left|\mathbb{E}_{h(x, y)}\left(W_{T} W_{T-h}\right)\right|=\left|\operatorname{Cov}\left(W_{T}, W_{T-h}\right)\right|$, pois $\mathbb{E}_{h(x, y)}\left(W_{t}\right)=0$ para todo $t=$ $1, \ldots, T$. 
Mas, de acordo com a desigualdade de Davydov ${ }^{4}$,

$$
\begin{array}{r}
\frac{2}{T} \sum_{\mathbf{k}}\left[\sum_{h=\eta+1}^{T-1}\left(1-\frac{h}{T}\right)\left|\mathbb{E}_{h(x, y)}\left(W_{T} W_{T-h}\right)\right|\right] \\
\frac{2}{T} \sum_{\mathbf{k}}\left[\sum_{h=\eta+1}^{T}\left(1-\frac{h}{T}\right) 2 \frac{r}{r-2}(2 \alpha(h))^{1-\frac{2}{r}}\left[\mathbb{E}_{h(x, y)}\left(\left|W_{T}\right|^{r}\right)\right]^{\frac{1}{r}}\left[\mathbb{E}_{h(x, y)}\left(\left|W_{T-h}\right|^{r}\right)\right]^{\frac{1}{r}}\right] .
\end{array}
$$

Mas, sabendo que $\left|W_{t}\right| \leq 2^{-l}$, para todo $(x ; y)$ e para todo $t=1, \ldots, T$, então $\left|W_{t}\right|^{r} \leq\left(2^{-l}\right)^{r}$, e assim $\mathbb{E}_{h(x, y)}\left(\left|W_{t}\right|^{r}\right) \leq 2^{-l r}$, para todo $t=1, \ldots, T$.

Logo,

$$
\begin{array}{r}
\frac{2}{T} \sum_{\mathbf{k}}\left[\sum_{h=\eta+1}^{T}\left(1-\frac{h}{T}\right) 2 \frac{r}{r-2}(2 \alpha(h))^{1-\frac{2}{r}}\left[\mathbb{E}_{h(x, y)}\left(\left|W_{T}\right|^{r}\right)\right]^{\frac{1}{r}}\left[\mathbb{E}_{h(x, y)}\left(\left|W_{T-h}\right|^{r}\right)\right]^{\frac{1}{r}}\right] \leq \\
\frac{1}{T} 2^{2 l} 2^{3-\frac{1}{r}} \frac{r}{r-2} \sum_{h=\eta+1}^{T}\left(1-\frac{h}{T}\right)(\alpha(h))^{1-\frac{2}{r}} 2^{-2 l} \leq \frac{1}{T} K_{r} \sum_{h=\eta+1}^{T}(\alpha(h))^{1-\frac{2}{r}}
\end{array}
$$

em que $K_{r}=2^{3-\frac{1}{r}} \frac{r}{r-2}$.

Mas, pela suposição $(\mathrm{S} 2), \sum_{h=\eta+1}^{T}(\alpha(h))^{1-\frac{2}{r}}=O\left(\eta^{-1}\right)$, então

$$
\frac{1}{T} K_{r} \sum_{h=\eta+1}^{T}(\alpha(h))^{1-\frac{2}{r}}=\frac{1}{T} K_{r} O\left(\eta^{-1}\right),
$$

concluindo a segunda parte de (4.5) e consequentemente a segunda parte de (4.4).

Sendo assim, considerando os resultados obtidos em (4.4) e (4.5), tem-se para a primeira parte de (4.3)

$$
\begin{aligned}
\mathbb{E}_{h(x, y)}\left\|\hat{C}_{l}(u ; v)-\mathbb{E}_{h(x, y)}\left(\hat{C}_{l}(u ; v)\right)\right\|_{2}^{2} & \leq \frac{\left(M_{1}\right)^{2}}{T}+\frac{2 M \eta}{T}-\frac{2 M}{T^{2}} \sum_{h=1}^{\eta} h \\
& +\frac{1}{T} K_{r} O\left(\eta^{-1}\right) .
\end{aligned}
$$

\footnotetext{
${ }^{4}$ Ver Apêndice A
} 
$1^{\mathrm{a}}$ parte de $(4.2)$ :

Considerando o estimador auxiliar, analogamente ao caso iid, tem-se que

$$
\mathbb{E}_{h(x, y)}\left\|\tilde{C}_{l}(u ; v)-\hat{C}_{l}(u ; v)\right\|_{2}^{2}=\sum_{\mathbf{k}} \mathbb{E}_{h(x, y)}\left[\left(\tilde{c}_{l, \mathbf{k}}-\hat{c}_{l, \mathbf{k}}\right)^{2}\right]
$$

Mas

$$
\begin{aligned}
{\left[\left(\tilde{c}_{l, \mathbf{k}}-\hat{c}_{l, \mathbf{k}}\right)^{2}\right] } & \leq\left(\left\|\Phi_{l, \mathbf{k}}(u ; v)\right\|_{\infty}\right)^{2}\left\{\frac{1}{T} \sum_{t=1}^{T}\left[\left|G\left(Y_{t}\right)-G_{T}\left(Y_{t}\right)\right|+\left|F\left(X_{t}\right)-F_{T}\left(X_{t}\right)\right|\right]\right\}^{2} \\
& =\left(\left\|\Phi_{l, \mathbf{k}}(u ; v)\right\|_{\infty}\right)^{2}\left\{\frac{1}{T} \sum_{t=1}^{T}\left[\left|\Delta\left(X_{t}\right)\right|+\left|\Delta\left(Y_{t}\right)\right|\right]\right\}^{2}
\end{aligned}
$$

e considerando $\Delta\left(Y_{i}\right)=\Delta\left(Y_{i}\right)\left(\mathbb{I}\left\{\left|\Delta\left(Y_{i}\right)\right|>\epsilon\right\}+\mathbb{I}\left\{\left|\Delta\left(Y_{i}\right)\right| \leq \epsilon\right\}\right)$, para $\epsilon>0$ fixado, em que $\epsilon=\frac{1}{n}$, tem-se que

$$
\begin{aligned}
\sum_{\mathbf{k}} \mathbb{E}_{h(x, y)}\left[\left(\tilde{c}_{l, \mathbf{k}}-\hat{c}_{l, \mathbf{k}}\right)^{2}\right] & \leq 2^{2 l}\left(\|\Phi(w ; z)\|_{\infty}\right)^{2} \sum_{\mathbf{k}}\left\{k_{1} \frac{1}{n^{2}}\right. \\
& \left.+\left(k_{2}+k_{3} \frac{1}{n}\right)\left[P\left\{\left|\Delta\left(X_{i}\right)\right|>\epsilon\right\}+P\left\{\left|\Delta\left(Y_{i}\right)\right|>\epsilon\right\}\right]\right\} .
\end{aligned}
$$

Como no caso iid, o termo dado em (4.6) é referente a aproximação das funções de distribuição pelas funções de distribuição empírica.

Neste caso, é necessário analisar como as funções podem ser aproximadas, considerando sequências de variáveis aleatórias associadas. Considerando os resultados apresentados em Yu (1993), em que para uma sequência $\left\{\mathbf{X}_{t}, t \in \mathbb{Z}\right\}$, com a mesma função de distribuição $F(x)$, se $F(x)$ é contínua e se

$$
\sum_{t=1}^{\infty} \frac{1}{T^{2}} \operatorname{Cov}\left\{X_{t}, S_{T-1}\right\}<\infty
$$

em que $t=1, \ldots, T$ e $S_{T}=\sum_{t=1}^{T} X_{t}$, então quando $T \rightarrow \infty$

$$
\sup _{x \in \mathbb{R}}\left|F_{T}(x)-F(x)\right| \stackrel{q \cdot c}{\longrightarrow} 0 .
$$


E supondo estacionariedade, a condição (4.7) pode ser substituída por

$$
\frac{1}{T} \sum_{t=1}^{T} \operatorname{Cov}\left\{X_{t}, X_{T}\right\} \longrightarrow 0 .
$$

Sendo assim, pela suposição (S4) e sabendo que a condição $\alpha$-mixing implica ergodicidade $^{4}$, por (4.8), tem-se que

$$
\sum_{\mathbf{k}} \mathbb{E}_{h(x, y)}\left[\left(\tilde{c}_{l, \mathbf{k}}-\hat{c}_{l, \mathbf{k}}\right)^{2}\right]=o(1),
$$

o que conclui a primeira parte de (4.2).

Logo, considerando os resultados obtidos em (4.2), tem-se que para (4.1)

$$
\begin{aligned}
\operatorname{MISE}\left(\tilde{C}_{l}(u ; v), C(u ; v)\right) & \leq \frac{\left(M_{1}\right)^{2}}{T}+\frac{2 M \eta}{T}-\frac{2 M}{T^{2}} \sum_{h=1}^{\eta} h \frac{1}{T} K_{r} O\left(\eta^{-1}\right) \\
& \leq K\left[\frac{2}{T}+M 2^{-2 l(s+2)}\right]
\end{aligned}
$$

o que garante o resultado.

\footnotetext{
${ }^{4}$ Ver Capítulo 2 para mais detalhes
} 
40 CAPÍTULO 4. ESTIMAÇÃO PARA O CASO DE SÉRIES TEMPORAIS 


\section{Capítulo 5}

\section{Simulações}

Neste capítulo, será apresentado o procedimento seguido para o estudo de simulação, além dos resultados obtidos para o caso iid e também para o caso dependente.

\subsection{Simulação para o caso de séries temporais}

Com o intuito de avaliar o método, e compará-lo a outros métodos já propostos, será utilizado o mesmo exemplo proposto por Fermanian and Scaillet (2003).

A simulação será feita a partir dos seguintes passos:

(1) Simule os dados a partir de um processo autorregressivo bivariado estacionário de ordem 1, dado por

$$
\mathbf{V}_{t}=\mathbf{A}+\mathbf{B V}_{t-1}+\epsilon_{t}
$$

em que $\mathbf{V}_{t}=\left(X_{t} ; Y_{t}\right), \epsilon_{t} \sim N(0 ; \boldsymbol{\Sigma}), \mathbf{A}=(1 ; 1)^{\prime}$. vec $(\mathbf{B})$ e vec $(\boldsymbol{\Sigma})$ são escolhidos de acordo com o tipo de componente.

Uma amostra de tamanho $n$ é gerada a partir do processo Gaussiano estacionário obtido de acordo com os valores adotados para $\operatorname{vec}(\mathbf{B})$ e $\operatorname{vec}(\boldsymbol{\Sigma})$. 
(2) Calcule a cópula empírica na grade de pontos $\left(\frac{i}{n}, \frac{j}{n}\right)$, em que

$$
\mathbb{C}_{n}\left(\frac{i}{n} ; \frac{j}{n}\right)=\frac{1}{n} \sum_{k=1}^{n} \mathbb{I}\left\{X_{k} \leq X_{(i)} ; Y_{k} \leq Y_{(j)}\right\} \text {, para } i, j=0,1, \ldots, n \text {. }
$$

(3) Calcule a estimativa da cópula utilizando o procedimento de estimação apresentado na seção a seguir, considerando o estimador dado em (3.6).

(4) Calcule o valor da cópula em cada ponto da grade como sendo $C\left(\frac{i}{n} ; \frac{j}{n}\right)=\frac{i j}{n^{2}}$.

(5) Repita os passos (1)-(3) $m$ vezes. Para cada ponto da grade, considerando as $m$ réplicas de cópulas estimadas e a cópula calculada no passo (4), calcule o Viés e o EQM como sendo

$$
\begin{aligned}
\text { Viés } & =\frac{1}{m} \sum_{p=1}^{m}\left(\hat{C}_{p}-C\right) \\
\mathrm{EQM} & =\frac{1}{m} \sum_{p=1}^{m}\left(\hat{C}_{p}-C\right)^{2},
\end{aligned}
$$

em que $\hat{C}_{p}$ é a estimativa da cópula para a $p$-ésima réplica.

\subsubsection{Procedimento de estimação}

Para estimar a cópula, de acordo com o estimador proposto, o procedimento é feito por meio dos seguintes passos:

(1) Encontre o inteiro $J$ tal que $N=2^{J} \leq \sqrt{n}<2^{J+1}$, $J$ representando o nível de resolução mais fino para uma amostra de tamanho $n$.

(2) Considerando o conjunto de dados, que neste contexto é uma matriz com valores entre 0 e 1 , há problemas de descontinuidade nas bordas. Como já mencionado ao longo do texto, um dos métodos para solucionar este problema é a simetrização dos dados, detalhado a seguir. Considere uma matriz $\mathbf{A}=\left(a_{p_{1} p_{2}}\right)$, com 
$p_{1}, p_{2} \in\{1, \ldots, p\}$. A idéia do método de simetrização é expandir a matriz $\mathbf{A}$ em uma matriz $\mathbf{B}$ de dimensão $(3 p \times 3 p)$, dada por

$$
\mathbf{B}=\left(\begin{array}{ccc}
{ }_{*} \mathbf{A}_{*} & { }_{*} \mathbf{A} & { }_{*} \mathbf{A}_{*} \\
\mathbf{A}_{*} & \mathbf{A} & \mathbf{A}_{*} \\
{ }_{*} \mathbf{A}_{*} & { }_{*} \mathbf{A} & { }_{*} \mathbf{A}_{*}
\end{array}\right) \text {, }
$$

em que ${ }_{*} \mathbf{A}_{*}=\left(a_{p+1-p_{1}, p+1-p_{2}}\right), \mathbf{A}_{*}=\left(a_{p_{1}, p+1-p_{2}}\right)$ e ${ }_{*} \mathbf{A}=\left(a_{p+1-p_{1}, p_{2}}\right)$.

A seguir, aplique o método e construa a matriz $\mathbf{B}$ de blocos a partir dos dados.

(3) Aplique a transformada discreta de ondaletas bidimensional em $B$, e então extraia o bloco de dimensão $(n \times n)$ de $\mathbf{B}$, na posição $(2,2)$. Dessa forma, são obtidas as estimativas dos coeficientes de $\tilde{c}_{l}$, nos níveis $l=J, J-1, J-2, \ldots, 1$.

(4) Aplique a transformada inversa de ondaletas, considerando os coeficientes estimados, obtendo a cópula estimada

$$
\tilde{C}_{l}(u ; v)=\sum_{\mathbf{k} \in \mathbb{Z}^{2}} \tilde{\alpha}_{l, \mathbf{k}} \Phi_{j, \mathbf{k}}(u ; v),(u ; v) \in(0,1) .
$$

(5) Selecione a cópula estimada $C^{*}=\tilde{C}_{l^{*}}$ como estimador da cópula $C$.

\subsubsection{Resultados}

\section{1 - Componentes Independentes}

Nesta seção, serão apresentados alguns resultados obtidos para o caso dependente, em que as componentes são independentes.

As simulações foram feitas utilizando o software Matlab R2012a.

Foram simuladas 5.000 réplicas para amostras de tamanho $n=1.024$ do processo autorregressivo dado em $(5.1), \operatorname{com} \operatorname{vec}(\mathbf{B})=(0,25 ; 0 ; 0 ; 0,75)^{\prime}$ e $\operatorname{vec}(\boldsymbol{\Sigma})=$ $(0,75 ; 0 ; 0 ; 1,25)^{\prime}$, o que resultou no processo Gaussiano estacionário dado por

$$
\left[\begin{array}{l}
X_{t} \\
Y_{t}
\end{array}\right] \sim N\left(\left[\begin{array}{l}
1,33 \\
4
\end{array}\right] ;\left[\begin{array}{ll}
0,8 & 0 \\
0 & 2,86
\end{array}\right]\right) .
$$


Para estes dados, foi proposto o uso da ondaleta Daubechie "D2". Para a correção da descontinuidade das bordas, foi utilizado o método da simetrização, detalhado na seção 5.1.1.

\begin{tabular}{|c|c|c|c|c|c|c|c|c|}
\hline \multicolumn{9}{|c|}{ Cópulas } \\
\hline & $\times 10^{-4}$ & $\mathrm{C}(, 01 ;, 01)$ & $\mathrm{C}(, 05 ;, 05)$ & $\mathrm{C}(, 25 ;, 25)$ & $\mathrm{C}(, 50 ;, 50)$ & $\mathrm{C}(, 75 ;, 75)$ & $\mathrm{C}(, 95 ;, 95)$ & $\mathrm{C}(, 99 ;, 99)$ \\
\hline & Real & 1,00 & 25,00 & 625,00 & $2.500,00$ & $5.625,00$ & $9.025,00$ & $9.801,00$ \\
\hline Nível & Estatística & \multicolumn{7}{|c|}{ Estimador Ondaleta "D2" (5000 réplicas) } \\
\hline \multirow{3}{*}{$l=1$} & Média & 1,79043 & 25,69 & 630,85 & $2.502,78$ & $5.624,26$ & $9.027,92$ & $9.851,29$ \\
\hline & Viés & 0,79043 & 0,69320 & 5,85242 & 2,78267 & $-0,73069$ & 2,92745 & 50,29317 \\
\hline & EQM & 0,00066 & 0,01840 & 0,42841 & 0,81192 & 0,42911 & 0,01671 & 0,25307 \\
\hline \multirow{3}{*}{$l=2$} & Média & 1,19 & 24,62 & 630,91 & $2.502,62$ & $5.624,21$ & $9.028,70$ & $9.814,31$ \\
\hline & Viés & 0,18936 & $-0,37974$ & 5,91207 & 2,62732 & $-0,78241$ & 3,70217 & 13,31907 \\
\hline & EQM & 0,00076 & 0,02233 & 0,41422 & 0,78837 & 0,41536 & 0,02264 & 0,017952 \\
\hline \multirow{3}{*}{$l=3$} & Média & 0,87626 & 24,51274 & 630,89 & $2.502,63$ & $5.624,32$ & $9.028,68$ & $9.807,86$ \\
\hline & Viés & $-0,12373$ & $-0,48726$ & 5,89316 & 2,62973 & $-0,67675$ & 3,68293 & 6,86647 \\
\hline & EQM & 0,00105 & 0,02195 & 0,40864 & 0,77857 & 0,40861 & 0,02208 & 0,00529 \\
\hline \multirow{3}{*}{$l=4$} & Média & 0,98333 & 24,50 & 630,88 & $2.502,66$ & $5.624,36$ & $9.028,58$ & $9.805,67$ \\
\hline & Viés & $-0,01667$ & $-0,49575$ & 5,88007 & 2,66045 & $-0,63316$ & 3,58812 & 4,67924 \\
\hline & EQM & 0,00114 & 0,02220 & 0,40662 & 0,77657 & 0,40668 & 0,02339 & 0,00329 \\
\hline \multirow{4}{*}{$l=5$} & Média & 0,98539 & 24,50 & 630,92 & $2.502,61$ & $5.624,35$ & $9.028,60$ & $9.805,69$ \\
\hline & Viés & $-0,01460$ & $-0,49638$ & 5,91641 & 2,61533 & $-0,64523$ & 3,59959 & 4,68638 \\
\hline & EQM & 0,00105 & 0,02250 & 0,41132 & 0,78310 & 0,410305 & 0,02330 & 0,00322 \\
\hline & & \multicolumn{7}{|c|}{ Estimador Ondaleta "D8"(1000 réplicas) - Morettin et al. (2010) } \\
\hline \multirow[t]{3}{*}{$\mathrm{J}=5$} & Viés & 0,66 & 1,31 & 1,76 & 11,59 & 16,16 & 3,38 & 9,27 \\
\hline & EQM & 0,00 & 0,03 & 0,42 & 0,42 & 0,40 & 0,02 & 0,01 \\
\hline & & \multicolumn{7}{|c|}{ Estimador Ondaleta "Haar"(500 réplicas) - Morettin et al. (2011) } \\
\hline \multirow[t]{3}{*}{$\mathrm{J}=5$} & Viés & 0,05 & 0,72 & 9,06 & 25,32 & 28,57 & 13,53 & 5,32 \\
\hline & EQM & 0,00 & 0,00 & 0,02 & 0,11 & 0,15 & 0,03 & 0,01 \\
\hline & & \multicolumn{7}{|c|}{ Estimador via Kernel (5000 réplicas) - Fermanian and Scaillet (2003) } \\
\hline \multirow[t]{2}{*}{ - } & Viés & $-0,09$ & $-0,08$ & 0,40 & 1,12 & $-0,90$ & $-0,04$ & 4,66 \\
\hline & EQM & 0,00 & 0,01 & 0,25 & 0,48 & 0,25 & 0,01 & 0,05 \\
\hline
\end{tabular}

Tabela 5.1: Média, Viés e EQM do estimador proposto utilizando Daubechie "D2" componentes independentes, com $\mathrm{n}=1024$.

Na Tabela 5.1 são apresentados os valores da média das réplicas dos valores estimados, além do Viés e do EQM, nos níveis de resolução $l=5, \ldots, 1$. Todos os valores são multiplos de $10^{-4}$. Também são apresentados os valores obtidos para os estima- 
dores propostos em Morettin et al. (2010), Morettin et al. (2011) e Fermanian and Scaillet (2003).

Pelos valores da Tabela 5.1, percebe-se que os níveis que apresentam melhores resultados são $l=4$ e $l=5$. Ambos apresentam também bons resultados quando comparados aos resultados apresentados por Morettin et al. (2010), Morettin et al. (2011) Fermanian and Scaillet (2003). Note também que considerando o estimador proposto em (3.6), são utilizados somente a função escala e seus respectivos coeficientes estimados.
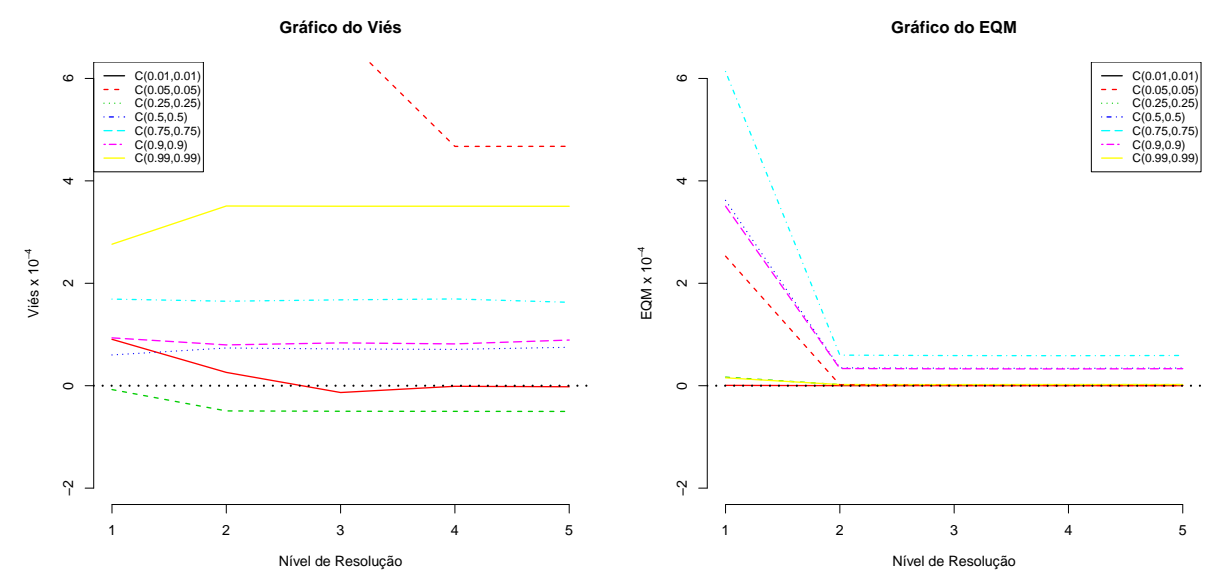

Figura 5.1: Gráficos do Viés e do EQM.

Na Figura 5.1 são apresentados os gráficos do Viés e do EQM das réplicas para as cópulas estimadas nos cinco níveis de resolução, $l=1,2, \ldots, 5$. Pela figura, percebe-se que há uma quedo dos valores do Viés e do EQM para níveis de resolução maiores.

Na Figura 5.2 são apresentados os gráficos das cópulas estimadas também em vários níveis. Pode-se perceber que são obtidos melhores resultados para os níveis $l=4$ e $l=5$. 

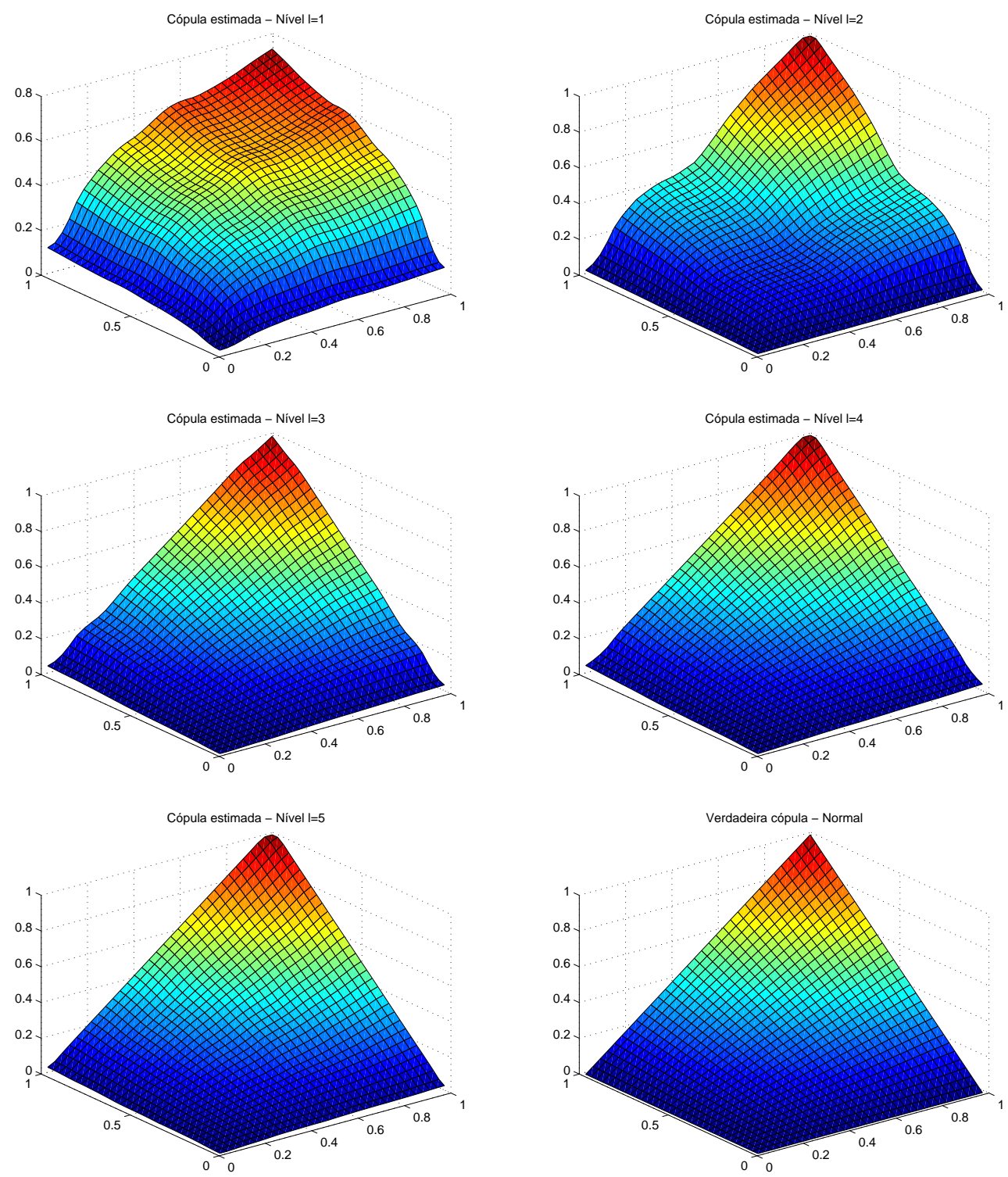

Figura 5.2: Gráficos das Cópulas estimadas em vários níveis.

\section{2 - Componentes Dependentes}

Para o caso em que as componentes são dependentes, será considerado o processo dado em $(5.1)$, em que vec $(\mathbf{B})=(0,25 ; 0,2 ; 0,2 ; 0,75)^{\prime}$ e vec $(\boldsymbol{\Sigma})=(0,75 ; 0,5 ; 0,5 ; 1.25)^{\prime}$. 
Neste caso, os componentes são dependentes e $C(u ; v)>u v$.

Assim, uma amostra de tamanho $n$ deve ser gerada a partir do processo Gaussiano estacionário

$$
\left[\begin{array}{l}
X_{t} \\
Y_{t}
\end{array}\right] \sim N\left(\left[\begin{array}{l}
3.05 \\
6.44
\end{array}\right] ;\left[\begin{array}{ll}
1.13 & 1.49 \\
1.49 & 3.98
\end{array}\right]\right)
$$

Como no caso de componentes independentes, foram geradas 5.000 réplicas para amostras de tamanho $n=1.024$, utilizando a distribuição dada em (5.2), propondo o uso da ondaleta Daubechie "D2". Logo, seguiu-se os mesmos passos de (1)-(5), detalhados na seção 5.1, além do procedimento detalhado na seção 5.1.1.

Os resultados são apresentados na Tabela 5.2, que apresenta valores do Viés e do EQM, múltiplos de $10^{-4}$. Todos os valores são bem próximos para todos os níveis de resolução. Porém, quando comparados com os valores obtidos por Morettin et al. (2010) apresentam valores maiores. Isso, pode ser devido ao fato de não utilizar as funções de detalhes, ou sequer um threshold que selecione os detalhes que seriam significantes na aproximação. Porém, quando comparados aos valores obtidos por Fermanian and Scaillet (2003), que utiliza kernels na estimação da cópula, os resultados são semelhantes.

Na Figura 5.3 são apresentados os gráficos das cópulas estimadas, no caso dependente, também nos cinco níveis.

Pelos gráficos, é possível perceber que, como mostrado pelas simulações, o que apresenta comportamento mais semelhante ao da verdadeira cópula é a cópula estimada via ondaletas com $l^{*}=5$.

Pelas simulações é possível perceber que o estimador, utilizando somente as funções escala, aparentemente apresenta melhores resultados para o caso iid. Logo, no caso dependente, é possível que as estimativas sejam influenciadas pela falta das funções de detalhes. Porém, as estimativas apresentam um número bem menor de coeficientes utilizados na estimação da cópula.

Visando verificar se o fato de não utilizar as funções de detalhes influencia muito no ajuste de cópulas via ondaletas, foram propostas simulações para o caso dependente, 


\begin{tabular}{|c|c|c|c|c|c|c|c|c|}
\hline \multicolumn{9}{|c|}{ Cópulas } \\
\hline & $\times 10^{-4}$ & $\mathrm{C}(, 01 ;, 01)$ & $\mathrm{C}(, 05 ;, 05)$ & $\mathrm{C}(, 25 ;, 25)$ & $\mathrm{C}(, 50 ;, 50)$ & $\mathrm{C}(, 75 ;, 75)$ & $\mathrm{C}(, 95 ;, 95)$ & $\mathrm{C}(, 99 ;, 99)$ \\
\hline & Real & 26,9 & 197,2 & $1.509,8$ & $3.739,9$ & $6.508,8$ & $9.197,2$ & $9.826,9$ \\
\hline Nível & Estatística & \multicolumn{7}{|c|}{ Estimador Ondaleta "D2" (5000 réplicas) } \\
\hline \multirow{3}{*}{$l=1$} & Média & 20,52574 & 172,051 & $1.376,47$ & $3.586,99$ & $6.465,42$ & $9.181,81$ & $9.855,91$ \\
\hline & Viés & 6,39937 & $-25,1177$ & $-132,371$ & $-152,891$ & $-43,4135$ & $-15,3651$ & 28,9833 \\
\hline & EQM & 0,01052 & 0,19315 & 2,20303 & 2,91158 & 0,54573 & 0,07141 & 0,08477 \\
\hline \multirow{3}{*}{$l=2$} & Média & 17,3965 & 165,603 & $1.374,81$ & $3.585,62$ & $6.465,04$ & $9.185,07$ & $9.821,37$ \\
\hline & Viés & $-9,52861$ & $-31,5727$ & $-134,02$ & $-154,26$ & $-43,7941$ & $-12,0981$ & $-5,55452$ \\
\hline & EQM & 0,02029 & 0,21037 & 2,22979 & 2,93473 & 0,54438 & 0,08432 & 0,00473 \\
\hline \multirow{3}{*}{$l=3$} & Média & 17,7891 & 164,393 & $1.374,55$ & $3.585,35$ & $6.465,20$ & $9.184,22$ & $9.817,49$ \\
\hline & Viés & $-9,13599$ & $-32,7832$ & $-134,28$ & $-154,53$ & $-43,6367$ & $-12,9511$ & $-9,43256$ \\
\hline & EQM & 0,01867 & 0,20959 & 2,23056 & 2,93672 & 0,55730 & 0,08182 & 0,014811 \\
\hline \multirow{3}{*}{$l=4$} & Média & 18,3893 & 164,139 & $1.374,60$ & $3.585,32$ & $6.465,16$ & $9.184,03$ & $9.815,85$ \\
\hline & Viés & $-8,53580$ & $-33,0367$ & $-134,233$ & $-154,568$ & $-43,6722$ & $-13,1460$ & $-11,0753$ \\
\hline & EQM & 0,022259 & 0,208256 & 2,242146 & 2,950065 & 0,558231 & 0,080473 & 0,022071 \\
\hline \multirow{4}{*}{$l=5$} & Média & 18,2113 & 164,098 & $1.374,62$ & $3.585,38$ & $6.465,17$ & $9.184,01$ & $9.815,71$ \\
\hline & Viés & $-8,71379$ & $-33,0777$ & $-134,221$ & $-154,503$ & $-43,6685$ & $-13,1644$ & $-11,2071$ \\
\hline & EQM & 0,021615 & 0,207158 & 2,240509 & 2,946415 & 0,560300 & 0,080146 & 0,021664 \\
\hline & & \multicolumn{7}{|c|}{ Estimador Ondaleta “D8” (1.000 réplicas) - Morettin et al. (2010) } \\
\hline \multirow[t]{3}{*}{$\mathrm{J}=5$} & Viés & $-0,30$ & 0,96 & 3,89 & 7,37 & 9,56 & 0,05 & 1,99 \\
\hline & EQM & 0,02 & 0,11 & 0,50 & 0,66 & 0,52 & 0,11 & 0,02 \\
\hline & & \multicolumn{7}{|c|}{ Estimador Ondaleta "Haar" (500 réplicas) - Morettin et al. (2011) } \\
\hline \multirow[t]{3}{*}{$\mathrm{J}=5$} & Viés & 0,60 & $-1,96$ & $-21,32$ & $-32,93$ & 13,27 & 9,22 & 16,75 \\
\hline & EQM & 0,02 & 0,11 & 0,46 & 0,73 & 0,50 & 0,11 & 0,05 \\
\hline & & \multicolumn{7}{|c|}{ Estimador via Kernel (5.000 réplicas) - Fermanian and Scaillet (2003) } \\
\hline \multirow[t]{2}{*}{-} & Viés & $-7,47$ & $-34,88$ & 130,32 & 172,28 & $-130,53$ & $-35,25$ & 7,65 \\
\hline & EQM & 0,01 & 0,18 & 1,98 & 3,36 & 1,99 & 0,18 & 0,01 \\
\hline
\end{tabular}

Tabela 5.2: Média, Viés e EQM do estimador proposto utilizando Daubechie "D2" componentes dependentes.

utilizando threshold. 

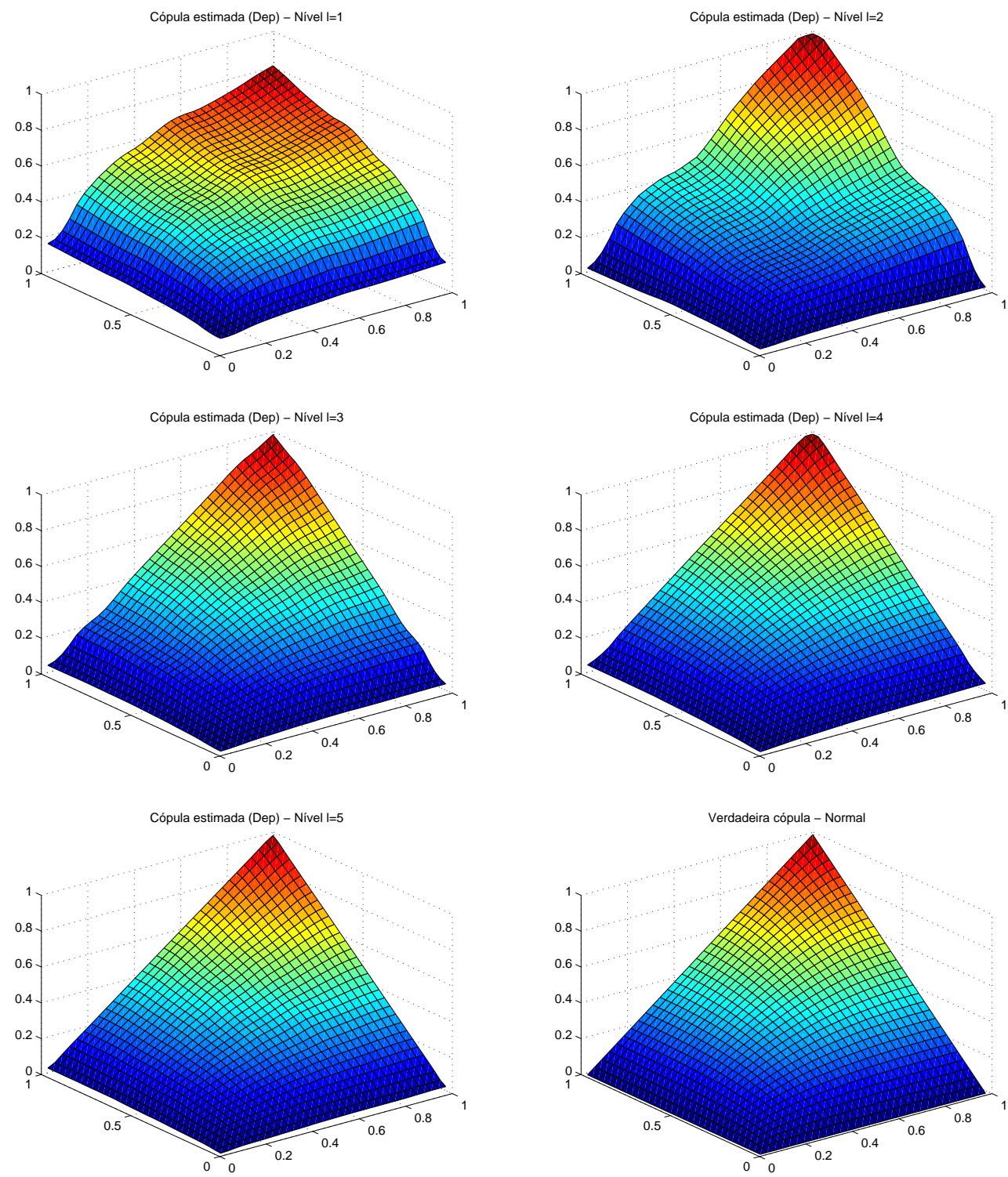

Figura 5.3: Gráficos das Cópulas estimadas em vários níveis para o componentes dependentes.

\subsection{Simulação para o caso de séries temporais com threshold}

Para introduzir o conceito de threshold na estimação de cópulas via ondaletas, considere a cópula estimada

$$
\hat{C}(u ; v)=\sum_{\mathbf{k}} \hat{c}_{l, \mathbf{k}} \Phi_{l, \mathbf{k}}(u ; v)+\sum_{j \geq l}^{J} \sum_{\mathbf{k} \in \mathbb{Z}^{2}} \sum_{\mu=h, v, d} \hat{d}_{j, \mathbf{k}}^{\mu} \Psi_{j, \mathbf{k}}^{\mu}(u ; v),
$$


em que $\hat{c}_{l, \mathbf{k}}$ e $\hat{d}_{j, \mathbf{k}}^{\mu}$ são estimados como o método proposto neste trabalho.

Para selecionar quais os coeficientes $\hat{d}_{j, \mathbf{k}}^{\mu}$ que seriam mais significantes ao ajuste, considera-se o threshold $\delta_{\lambda}($.$) , em que \lambda$ é o parâmetro que indica o critério de escolha.

Geralmente são utilizados os thresholds definidos como Hard e Soft threshold, dados por

$$
\delta_{\lambda}^{H}(x)=\left\{\begin{array}{l}
0, \text { se }|x| \leq \lambda \\
x, \text { se }|x|>\lambda
\end{array}\right.
$$

$\mathrm{e}$

$$
\delta_{\lambda}^{S}(x)=\left\{\begin{array}{l}
0, \text { se }|x| \leq \lambda, \\
\sin (x)(|x|-\lambda), \text { se }|x|>\lambda
\end{array}\right.
$$

Para a escolha do valor mais adequado para $\lambda$, são propostos alguns estimadores, como pode ser visto em Morettin (2014).

Neste trabalho, foi proposto o uso do threshold para altos quantis, como sugerido em Morettin et al. (2010), em que

$$
\delta_{Q}(x)=\left\{\begin{array}{l}
0, \text { se } x \leq Q_{p}(\boldsymbol{x}) \\
x, \text { se } x>Q_{p}(\boldsymbol{x})
\end{array}\right.
$$

em que $Q_{p}(\boldsymbol{x})$ é o p-quantil de $\boldsymbol{x}$.

Logo, para o estudo de simulação foram geradas 5.000 réplicas para amostras de tamanho 1.024, considerando dados de séries temporais, com componentes dependentes e propondo o uso da ondaleta Daubechie "D2" para os ajustes. Seguindo os mesmos passos do algoritmo detalhado, porém considerando um threshold de alto quantil com $p=0.9$ para os coeficientes $\hat{d}_{j, \mathbf{k}}^{\mu}$, foram calculados os valores do viés e do EQM, todos multiplos de $10^{-4}$, como podem ser vistos na tabela a seguir.

De acordo com os valores apresentados na Tabela 5.3 é possível notar que não houve muitas alterações para os valores do Viés e do EQM calculados para as cópulas ajustadas em valores das bordas. Já para outros pontos, a tabela apresenta menores valores para o Viés e para o EQM quando utilizou-se o threshold, indicando que para 


\subsection{SIMULAÇÃO PARA O CASO DE SÉRIES TEMPORAIS COM THRESHOLD51}

\begin{tabular}{|c|c|c|c|c|c|c|c|c|}
\hline \multicolumn{9}{|c|}{ Cópulas } \\
\hline & $\times 10^{-4}$ & $\mathrm{C}(, 01 ;, 01)$ & $\mathrm{C}(, 05 ;, 05)$ & $\mathrm{C}(, 25 ;, 25)$ & $\mathrm{C}(, 50 ;, 50)$ & $\mathrm{C}(, 75 ;, 75)$ & $\mathrm{C}(, 95 ;, 95)$ & $\mathrm{C}(, 99 ;, 99)$ \\
\hline & Real & 26,9 & 197,2 & $1.509,8$ & $3.739,9$ & $6.508,8$ & $9.197,2$ & $9.826,9$ \\
\hline Nível & Estatística & \multicolumn{7}{|c|}{ Estimador Ondaleta "D2" - com threshold(5000 réplicas) } \\
\hline \multirow{3}{*}{$l=1$} & Média & 20,52574 & 184,39444 & $1.482,05359$ & $3.656,44652$ & $6.518,90115$ & $9.181,81132$ & $9.822,95164$ \\
\hline & Viés & $-6,39936$ & $-12,78198$ & $-26,78521$ & $-83,44168$ & 10,062352 & $-15,36508$ & $-3,97346$ \\
\hline & EQM & 0,01052 & 0,10116 & 0,47938 & 1,22934 & 0,37617 & 0,07141 & 0,00726 \\
\hline \multirow{3}{*}{$l=2$} & Média & 17,3965 & 185,1919 & $1.483,5674$ & $3.656,2255$ & $6.517,9895$ & $9.185,0776$ & $9.821,3706$ \\
\hline & Viés & $-9,52861$ & $-11,98454$ & $-25,27142$ & $-83,66274$ & 9,15075 & $-12,09881$ & $-5,55453$ \\
\hline & EQM & 0,02029 & 0,11018 & 0,50373 & 1,23807 & 0,361494 & 0,08432 & 0,00473 \\
\hline \multirow{3}{*}{$l=3$} & Média & 17,78912 & 185,72434 & $1.483,40881$ & $3.656,31813$ & $6.518,07051$ & $9.184,22524$ & $9.817,49253$ \\
\hline & Viés & $-9,13599$ & $-11,45208$ & $-25,42999$ & $-83,57007$ & 9,23171 & $-12,95116$ & $-9,43256$ \\
\hline & EQM & 0,01867 & 0,12077 & 0,50173 & 1,24342 & 0,36941 & 0,08182 & 0,01481 \\
\hline \multirow{3}{*}{$l=4$} & Média & 18,38931 & 185,65573 & $1.483,51320$ & $3.656,32124$ & $6.518,10266$ & $9.184,03034$ & $9.815,84980$ \\
\hline & Viés & $-8,53580$ & $-11,52069$ & $-25,32560$ & $-83,56696$ & 9,26386 & $-13,14606$ & $-11,07530$ \\
\hline & EQM & 0,02225 & 0,11923 & 0,50913 & 1,25208 & 0,37141 & 0,08047 & 0,02207 \\
\hline \multirow{4}{*}{$l=5$} & Média & 18,21132 & 185,73713 & $1.483,50617$ & $3.656,31393$ & $6.518,09973$ & $9.184,01194$ & $9.815,71797$ \\
\hline & Viés & $-8,71379$ & $-11,43929$ & $-25,33263$ & $-83,57427$ & 9,26093 & $-13,16446$ & $-11,20713$ \\
\hline & EQM & 0,02161 & 0,12264 & 0,50845 & 1,25159 & 0,37387 & 0,08014 & 0,02166 \\
\hline & & \multicolumn{7}{|c|}{ Estimador Ondaleta "D8” (1.000 réplicas) - Morettin et al. (2010) } \\
\hline \multirow[t]{3}{*}{$\mathrm{J}=5$} & Viés & $-0,30$ & 0,96 & 3,89 & 7,37 & 9,56 & 0,05 & 1,99 \\
\hline & EQM & 0,02 & 0,11 & 0,50 & 0,66 & 0,52 & 0,11 & 0,02 \\
\hline & & \multicolumn{7}{|c|}{ Estimador Ondaleta "Haar" (500 réplicas) - Morettin et al. (2011) } \\
\hline \multirow[t]{3}{*}{$\mathrm{J}=5$} & Viés & 0,60 & $-1,96$ & $-21,32$ & $-32,93$ & 13,27 & 9,22 & 16,75 \\
\hline & EQM & 0,02 & 0,11 & 0,46 & 0,73 & 0,50 & 0,11 & 0,05 \\
\hline & & \multicolumn{7}{|c|}{ Estimador via Kernel (5.000 réplicas) - Fermanian and Scaillet (2003) } \\
\hline \multirow[t]{2}{*}{-} & Viés & $-7,47$ & $-34,88$ & 130,32 & 172,28 & $-130,53$ & $-35,25$ & 7,65 \\
\hline & EQM & 0,01 & 0,18 & 1,98 & 3,36 & 1,99 & 0,18 & 0,01 \\
\hline
\end{tabular}

Tabela 5.3: Média, Viés e EQM do estimador proposto utilizando Daubechie "D2" caso dependente com threshold de quantil com $p=0.9$.

dados dependentes, seria mais adequado o ajuste da cópula via ondaletas utilizando também as funções de detalhes.

Na Figura 5.4 são apresentados os gráficos das cópulas estimadas em vários níveis para o caso de séries temporais com threshold. Pelos gráficos é possível notar que realmente há uma melhora quando comparados aos gráficos da Figura 5.3, pois todas as figuras em todos os níveis são parecidas com a verdadeira cópula. Como são utiliza- 

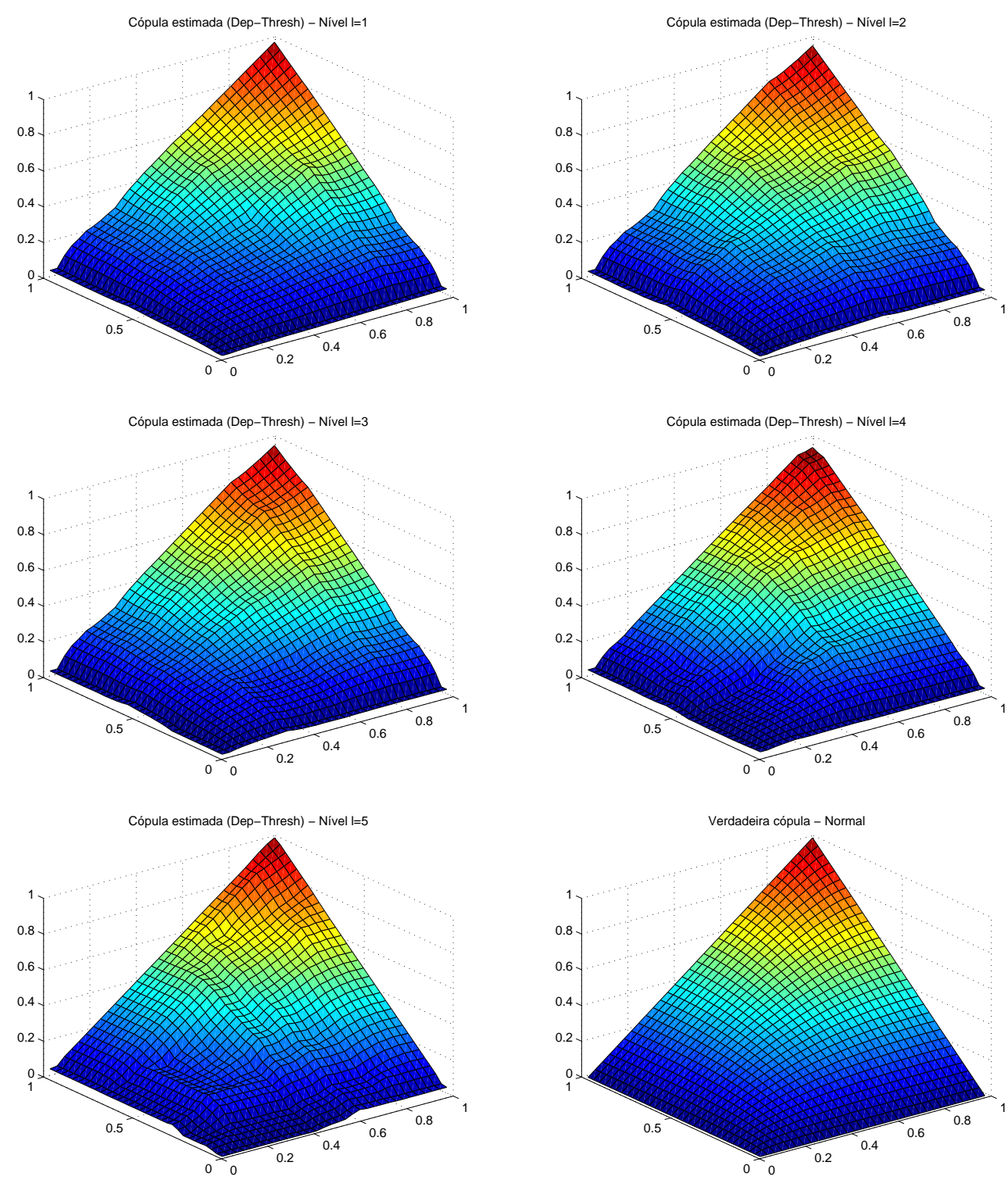

Figura 5.4: Gráficos das Cópulas estimadas em vários níveis para o componentes dependentes com Threshold.

das as funções de detalhes além da função escala, as cópulas estimadas com threshold parecem ser menos suaves, porém com um Viés e EQM menores quando comparadas 
as cópulas estimadas no caso de séries temporais com componentes dependente sem threshold.

Este método de estimação com uso de threshold também é chamado de método não linear, uma vez que há uma seleção de coeficientes utilizados no ajuste. Estes estimadores não lineares para cópulas seriam uma extensão do estimador proposto, uma vez que seria necessário novos cálculos de taxa de convergência para demonstrar a eficiência do estimador. Esta estensão não será apresentada neste trabalho, mas poderia ser uma sugestão para trabalhos futuros.

\subsection{Simulação para o caso iid}

Foram feitas simulações também para o caso em que a amostra é iid, considerando componentes dependentes e independentes, visando analisar o comportamento do estimador.

\section{1 - Componentes Independentes}

Para este caso, serão consideradas as variáveis aleatórias $(X, Y)$ tais que

$$
\left[\begin{array}{l}
X \\
Y
\end{array}\right] \sim N\left(\left[\begin{array}{l}
1,33 \\
4
\end{array}\right] ;\left[\begin{array}{ll}
0,8 & 0 \\
0 & 2,86
\end{array}\right]\right)
$$

Foram geradas 5.000 réplicas de uma amostra de tamanho $n=1.024$ obtida da densidade dada em (5.3). Foi proposto, assim como no caso dependente, o uso da ondaleta Daubechie "D2". A partir dos passos detalhados nas seções 5.1 e 5.2, foram obtidos os valores do Viés e do EQM, mútliplos de $10^{-4}$ apresentados na Tabela 5.4.

Os valores da Tabela 5.4 indicam que quando o nível $l$ aumenta, os valores do Viés e do EQM tendem a diminuir, indicando que os níveis $l=4$ e $l=5$ apresentam melhores resultados.

Considerando que os valores são múltiplos de $10^{-4}$, pode-se concluir que a estimação da função cópula, via ondaletas para dados independentes com componentes 


\begin{tabular}{|c|c|c|c|c|c|c|c|c|}
\hline \multicolumn{9}{|c|}{ Cópulas } \\
\hline & $\times 10^{-4}$ & $\mathrm{C}(, 01 ;, 01)$ & $\mathrm{C}(, 05 ;, 05)$ & $\mathrm{C}(, 25 ;, 25)$ & $\mathrm{C}(, 50 ;, 50)$ & $\mathrm{C}(, 75 ;, 75)$ & $\mathrm{C}(, 95 ;, 95)$ & $\mathrm{C}(, 99 ;, 99)$ \\
\hline & Real & 1,00 & 25,00 & 625,00 & $2.500,00$ & $5.625,00$ & $9.025,00$ & $9.801,00$ \\
\hline Nível & Estatística & \multicolumn{7}{|c|}{ Estimador Ondaleta "D2" (5000 réplicas) } \\
\hline \multirow{3}{*}{$l=1$} & Média & 1,90561 & 24,92 & 625,60 & $2.501,69$ & $5.625,93$ & $9.027,76$ & $9.851,28$ \\
\hline & Viés & 0,90561 & $-0,07571$ & 0,60058 & 1,69088 & 0,93426 & 2,76271 & 50,2832 \\
\hline & EQM & 0,00622 & 0,16637 & 3,6186 & 6,13468 & 3,50512 & 0,15379 & 2,52971 \\
\hline \multirow{3}{*}{$l=2$} & Média & 1,25984 & 24,50 & 625,74 & $2.501,65$ & $5.625,80$ & $9.028,50$ & $9.814,29$ \\
\hline & Viés & 0,25984 & $-0,49238$ & 0,73695 & 1,64967 & 0,79662 & 3,50903 & 13,2999 \\
\hline & EQM & 0,00071 & 0,02078 & 0,34866 & 0,59704 & 0,33520 & 0,02173 & 0,01788 \\
\hline \multirow{3}{*}{$l=3$} & Média & 0,86686 & 24,50 & 625,72 & $2.501,67$ & $5.625,84$ & $9.028,50$ & $9.807,87$ \\
\hline & Viés & $-0,13313$ & $-0,49926$ & 0,71719 & 1,67639 & 0,83611 & 3,50460 & 6,87077 \\
\hline & EQM & 0,00099 & 0,02047 & 0,34133 & 0,58830 & 0,32950 & 0,02113 & 0,00531 \\
\hline \multirow{3}{*}{$l=4$} & Média & 0,98855 & 24,49925 & 625,70 & $2.501,69$ & $5.625,8162$ & $9.028,50$ & $9.805,67$ \\
\hline & Viés & $-0,01144$ & $-0,50075$ & 0,70948 & 1,69390 & 0,81623 & 3,50504 & 4,67279 \\
\hline & EQM & 0,001062 & 0,02078 & 0,33915 & 0,58574 & 0,32783 & 0,02260 & 0,00327 \\
\hline \multirow{3}{*}{$l=5$} & Média & 0,97892 & 24,50 & 625,75 & $2.501,62$ & $5.625,89$ & $9.028,50$ & $9.805,67$ \\
\hline & Viés & $-0,02107$ & 0,50252 & 0,75044 & 1,62857 & 0,89153 & 3,50348 & 4,67398 \\
\hline & EQM & 0,00095 & 0,02120 & 0,34447 & 0,59008 & 0,33201 & 0,02246 & 0,00318 \\
\hline
\end{tabular}

Tabela 5.4: Média, Viés e EQM do estimador proposto utilizando Daubechie "D2" caso iid com componentes independentes.

independentes apresentou resultado satisfatório.

\section{2 - Componentes Dependentes}

Considerando componentes dependentes, foram geradas 5.000 réplicas de uma amostra de tamanho $n=1.024$ obtida da densidade conjunta

$$
\left[\begin{array}{l}
X \\
Y
\end{array}\right] \sim N\left(\left[\begin{array}{l}
3.05 \\
6.44
\end{array}\right] ;\left[\begin{array}{ll}
1.13 & 1.49 \\
1.49 & 3.98
\end{array}\right]\right)
$$

Os resultados são apresentados na Tabela 5.5, indicando comportamento semelhante ao dos valores obtidos no caso dependente. Como comentado anteriormente, isso pode ser pelo fato de não utilizarmos as funções de detalhes na aproximação, fato evidenciado quando a estimação da cópula foi feita utilizando threshold no caso 
dependente.

\begin{tabular}{|c|c|c|c|c|c|c|c|c|}
\hline \multicolumn{9}{|c|}{ Cópulas } \\
\hline & $\times 10^{-4}$ & $\mathrm{C}(, 01 ;, 01)$ & $\mathrm{C}(, 05 ;, 05)$ & $\mathrm{C}(, 25 ;, 25)$ & $\mathrm{C}(, 50 ;, 50)$ & $\mathrm{C}(, 75 ;, 75)$ & $\mathrm{C}(, 95 ;, 95)$ & $\mathrm{C}(, 99 ;, 99)$ \\
\hline & Real & 26,9 & 197,2 & $1.509,8$ & $3.739,9$ & $6.508,8$ & $9.197,2$ & $9.826,9$ \\
\hline Nível & Estatística & \multicolumn{7}{|c|}{ Estimador Ondaleta "D2" (5000 réplicas) } \\
\hline \multirow{3}{*}{$l=5$} & Média & 28,02 & 246,54 & $1.693,19$ & $3.974,67$ & $6.689,60$ & $9.247,27$ & $9.839,80$ \\
\hline & Viés & 5,547882 & 24,68301 & 92,17984 & 117,39426 & 90,38550 & 25,04703 & 6,43748 \\
\hline & EQM & 0,025848 & 0,33976 & 3,78879 & 6,04942 & 3,66830 & 0,34989 & 0,03637 \\
\hline \multirow{3}{*}{$l=4$} & Média & 28,24 & 246,79 & $1.693,09$ & $3.974,68$ & $6.689,39$ & $9.247,31$ & $9.840,41$ \\
\hline & Viés & 5,65745 & 24,80838 & 92,12631 & 117,39958 & 90,27855 & 25,07040 & 6,74231 \\
\hline & EQM & 0,02690 & 0,34450 & 3,78089 & 6,04487 & 3,65641 & 0,35090 & 0,03949 \\
\hline \multirow{3}{*}{$l=3$} & Média & 29,29 & 247,85 & $1.693,24$ & $3.974,8053$ & $6.689,53$ & $9.246,78$ & $9.840,04$ \\
\hline & Viés & 6,18244 & 25,34008 & 92,20058 & 117,45853 & 90,34739 & 24,80288 & 6,55926 \\
\hline & EQM & 0,03151 & 0,36085 & 3,78883 & 6,05331 & 3,66433 & 0,33809 & 0,02950 \\
\hline \multirow{3}{*}{$l=2$} & Média & 28,46279 & 253,11 & $1.694,23$ & $3.975,69$ & $6.690,42$ & $9.247,86$ & $9.835,70$ \\
\hline & Viés & 5,76884 & 27,96876 & 92,69756 & 117,90462 & 90,79356 & 25,34236 & 4,39136 \\
\hline & EQM & 0,01991 & 0,42927 & 3,83516 & 6,10562 & 3,70585 & 0,35227 & 0,01050 \\
\hline \multirow{3}{*}{$l=1$} & Média & 27,77 & 235,93 & $1.699,63$ & $3.979,61$ & $6.694,94$ & $9.241,76$ & $9.865,37$ \\
\hline & Viés & 5,42284 & 19,37992 & 95,39718 & 119,8644 & 93,05555 & 22,29249 & 19,22409 \\
\hline & EQM & 0,01437 & 0,21517 & 4,06349 & 6,31467 & 3,89012 & 0,26519 & 0,14911 \\
\hline
\end{tabular}

Tabela 5.5: Média, Viés e EQM do estimador proposto utilizando Daubechie "D2" caso iid com componentes dependentes. 


\subsection{Simulações adicionais}

Durante o estudo de simulação feito para este trabalho, foi observado que utilizar somente a função de escala no processo de estimação da cópula, para dados dependentes, pode fazer com que o ajuste não seja tão preciso. Porém, como pode ser visto, o Viés e o EQM foi calculado de forma pontual.

Visando obter uma outra análise da qualidade do ajuste, foi utilizado o mesmo procedimento que o utilizado em Autin et al. (2010), em que as cópulas ajustadas via ondaletas são comparadas com algumas cópulas paramétricas.

Considere uma função de perda empírica definida por

$\operatorname{Erro}\left(\hat{C}_{l^{*}}, C_{\theta}\right)=\frac{1}{N^{2}}\left\|\hat{C}_{l^{*}}-C_{\theta}\right\|_{2}^{2}=\frac{1}{N^{2}} \sum_{i=1}^{N} \sum_{j=1}^{N}\left\{\hat{C}_{l^{*}}\left(\frac{i}{N}, \frac{j}{N}\right)-C_{\theta}\left(\frac{i}{N}, \frac{j}{N}\right)\right\}^{2}$,

denominado como Erro entre as cópulas, em que $N$ é o tamanho da grade, $\theta$ é o parâmetro da verdadeira cópula e $\hat{C}_{l^{*}}$ é a cópula ajustada via ondaletas.

Também foi calculado o erro relativo (ER) definido por

$$
\operatorname{ER}\left(\hat{C}_{l^{*}}, C_{\theta}\right)=\operatorname{Erro}\left(\hat{C}_{l^{*}}, C_{\theta}\right) \times\left[\frac{1}{N^{2}}\left\|C_{\theta}\right\|_{2}^{2}\right]^{-1}
$$

Foram consideradas então 5.000 réplicas de amostras de tamanhos $n=256$ e $n=1.024$, obtidas das cópulas Normal, t-Student, Gumbel, Clayton e Frank, com parâmetros fixos. Para a cópula normal, o parâmetro fixado é o coeficiente de correlação, para a t-Student são o coeficiente de correlação e os graus de liberdade, para as cópulas Gumbel, Clayton e Frank, denominadas cópulas arquimedianas, o parâmetro fixado é o que mede o grau de dependência entre as séries, utilizado na função geradora das cópulas arquimedianas. Para mais detalhes, veja Nelsen (2005).

O procedimento utilizado é detalhado a seguir:

(1) Gere uma amostra de tamanho $n$ de cada cópula paramétrica;

(2) Estime a cópula via ondaletas; 
(3) Calcule o $E R$ entre a cópula paramétrica e a estimada;

(4) Repita os passos (1)-(3) r-vezes;

(5) Calcule a média e o desvio padrão entre as r-ésimas réplicas do $E R$;

Para cada uma das réplicas, foram ajustadas as cópulas via ondaletas, considerando algumas ondaletas Daubechies, e para cada réplica foram calculados os erros e os erros relativos em relação a verdadeira cópula. Logo, calculou-se a média e o desvio padrão para os erros relativos.

Os valores obtidos, todos múltiplos de $10^{-4}$, são apresentados nas Tabelas 5.6 e 5.7 , para amostras de tamanho $n=256$ e $n=1.024$, respectivamente.

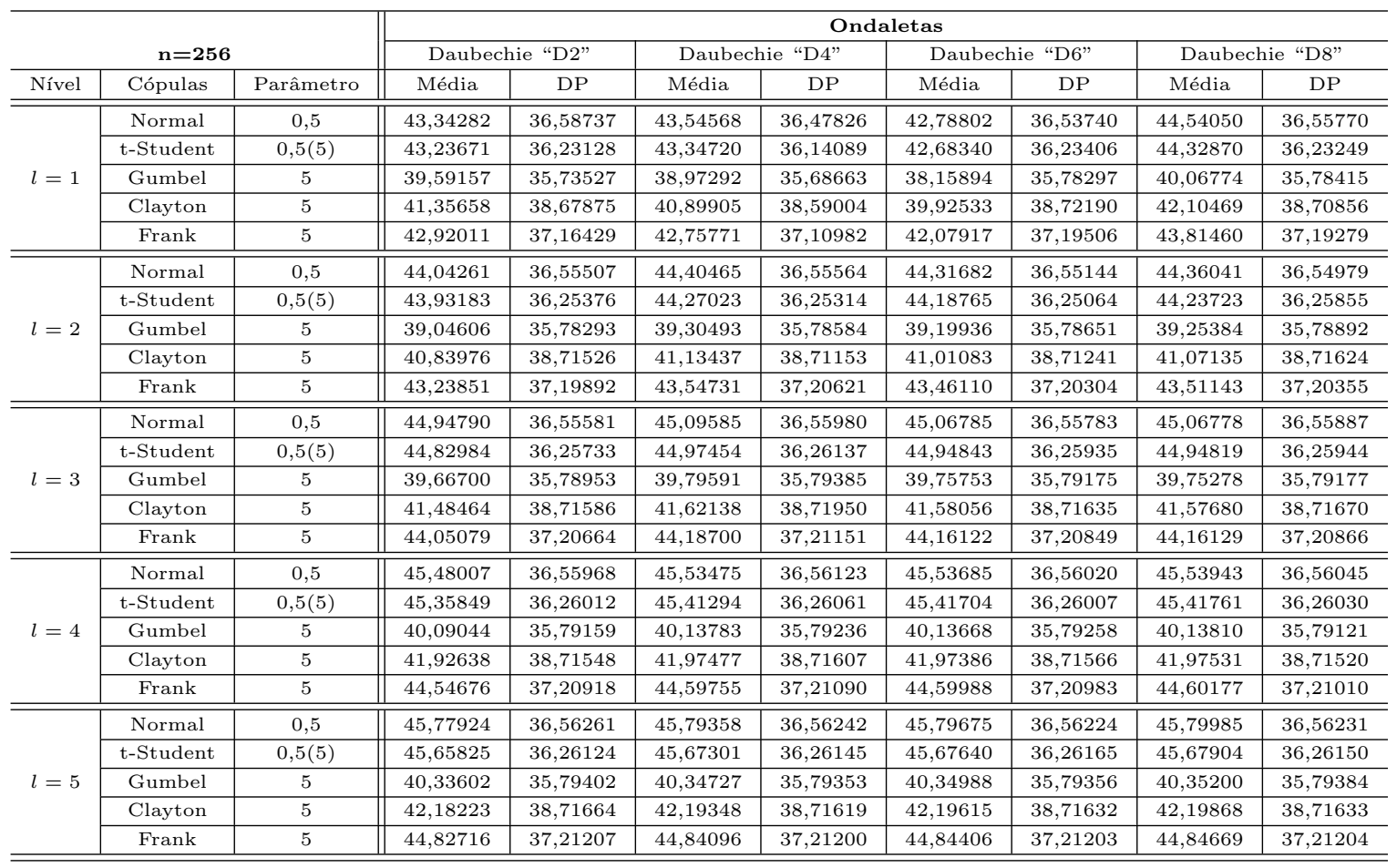

Tabela 5.6: Média e desvio padrão do erro relativo entre as cópulas estimadas e as cópulas verdadeiras. 
De acordo com os valores apresentados na Tabela 5.6 é possível notar que os valores não se alteram muito quando adotados outros tipos de ondaletas Daubechies. Os valores também não se alteram muito para os diferentes valores de $l^{*}$. Para os diferentes tipos de cópulas, a que apresentou menor valor para a média dos erros relativos foi a cópula Gumbel. De acordo com Autin et al. (2010), as cópulas que apresentam menores valores para o erro relativo seriam as que possuem maior regularidade.

\begin{tabular}{|c|c|c|c|c|c|c|c|c|c|c|}
\hline & & & \multicolumn{8}{|c|}{ Ondaletas } \\
\hline \multicolumn{3}{|c|}{$\mathrm{n}=1.024$} & \multicolumn{2}{|c|}{ Daubechie "D2" } & \multicolumn{2}{|c|}{ Daubechie "D4" } & \multicolumn{2}{|c|}{ Daubechie "D6" } & \multicolumn{2}{|c|}{ Daubechie "D8" } \\
\hline Nível & Cópulas & Parâmetro & Média & DP & Média & DP & Média & DP & Média & DP \\
\hline \multirow{5}{*}{$l=1$} & Normal & 0,5 & 11,03417 & 8,63750 & 11,05499 & 8,63688 & 11,04717 & 8,63676 & 11,07887 & 8,63736 \\
\hline & t-Student & $0,5(5)$ & 11,29598 & 9,16735 & 11,31518 & 9,16635 & 11,30857 & 9,16726 & 11,33834 & 9,16672 \\
\hline & Gumbel & 5 & 9,81370 & 8,89528 & 9,82328 & 8,89501 & 9,81392 & 8,89545 & 9,84704 & 8,89538 \\
\hline & Clayton & 5 & 10,42943 & 9,51825 & 10,43882 & 9,51825 & 10,42894 & 9,51884 & 10,46558 & 9,51880 \\
\hline & Frank & 5 & 10,95613 & 9,26732 & 10,97186 & 9,26754 & 10,96547 & 9,26763 & 10,99454 & 9,26772 \\
\hline \multirow{5}{*}{$l=2$} & Normal & 0,5 & 11,14503 & 8,63724 & 11,16010 & 8,63733 & 11,16077 & 8,63740 & 11,16245 & 8,63724 \\
\hline & t-Student & $0,5(5)$ & 11,40716 & 9,16740 & 11,42161 & 9,16740 & 11,42249 & 9,16748 & 11,42419 & 9,16737 \\
\hline & Gumbel & 5 & 9,89338 & 8,89563 & 9,90560 & 8,89577 & 9,90529 & 8,89578 & 9,90719 & 8,89578 \\
\hline & Clayton & 5 & 10,51101 & 9,51900 & 10,52401 & 9,51886 & 10,52369 & 9,51868 & 10,52556 & 9,51886 \\
\hline & Frank & 5 & 11,05746 & 9,26775 & 11,07062 & 9,26748 & 11,07133 & 9,26762 & 11,07316 & 9,26775 \\
\hline \multirow{5}{*}{$l=3$} & Normal & 0,5 & 11,20998 & 8,63756 & 11,21698 & 8,63761 & 11,21753 & 8,63762 & 11,21796 & 8,63762 \\
\hline & t-Student & $0,5(5)$ & 11,47195 & 9,16743 & 11,47882 & 9,16745 & 11,47943 & 9,16743 & 11,47988 & 9,16745 \\
\hline & Gumbel & 5 & 9,94526 & 8,89593 & 9,95110 & 8,89594 & 9,95134 & 8,89591 & 9,95163 & 8,89589 \\
\hline & Clayton & 5 & 10,56532 & 9,51891 & 10,57147 & 9,51894 & 10,57170 & 9,51892 & 10,57202 & 9,51892 \\
\hline & Frank & 5 & 11,11776 & 9,26758 & 11,12417 & 9,26759 & 11,12474 & 9,26759 & 11,12514 & 9,26761 \\
\hline \multirow{5}{*}{$l=4$} & Normal & 0,5 & 11,24418 & 8,63769 & 11,24728 & 8,63771 & 11,24783 & 8,63770 & 11,24809 & 8,63772 \\
\hline & t-Student & $0,5(5)$ & 11,50619 & 9,16751 & 11,50928 & 9,16752 & 11,50986 & 9,16752 & 11,51010 & 9,16753 \\
\hline & Gumbel & 5 & 9,97319 & 8,89596 & 9,97580 & 8,89595 & 9,97618 & 8,89595 & 9,97640 & 8,89597 \\
\hline & Clayton & 5 & 10,59459 & 9,51895 & 10,59726 & 9,51897 & 10,59770 & 9,51896 & 10,59791 & 9,51895 \\
\hline & Frank & 5 & 11,14971 & 9,26764 & 11,15258 & 9,26766 & 11,15312 & 9,26766 & 11,15336 & 9,26765 \\
\hline \multirow{5}{*}{$l=5$} & Normal & 0,5 & 11,52501 & 8,63776 & 11,26400 & 8,63775 & 11,26434 & 8,63776 & 11,26448 & 8,63776 \\
\hline & t-Student & $0,5(5)$ & 11,52500 & 9,16757 & 11,52610 & 9,16757 & 11,52639 & 9,16757 & 11,52655 & 9,16757 \\
\hline & Gumbel & 5 & 9,98861 & 8,89598 & 9,98950 & 8,89599 & 9,98975 & 8,89597 & 9,98987 & 8,89598 \\
\hline & Clayton & 5 & 10,61070 & 9,51895 & 10,61160 & 9,51896 & 10,61188 & 9,51895 & 10,61202 & 9,51895 \\
\hline & Frank & 5 & 11,16720 & 9,26767 & 11,16830 & 9,26767 & 11,16859 & 9,26767 & 11,16874 & 9,26767 \\
\hline
\end{tabular}

Tabela 5.7: Média e desvio padrão do erro relativo entre as cópulas estimadas e as cópulas verdadeiras.

Os valores apresentados na Tabela 5.7 são semelhantes aos apresentados na Tabela 5.6 em relação a utilização de diferentes tipos de ondaletas e para os diferentes níveis, porém a média e o desvio padrão apresentam valores menores, indicando que para maiores valores de $n$, os erros relativos tendem a ser menores. 


\section{Capítulo 6}

\section{Aplicações}

Neste capítulo serão apresentadas aplicações para dados reais considerando o estimador via ondaletas, para os casos independente e de séries temporais.

\subsection{Casos de séries temporais}

Para séries temporais, as aplicações foram divididas em três partes, considerando seis séries, analisadas duas a duas, para as quais foram ajustadas 3 cópulas.

Com o intuito de avaliar o desempenho do estimador proposto, foram utilizadas as mesmas séries que em Morettin et al. (2010).

\subsubsection{Séries CAC40 - DAX30}

A série CAC40, abreviação de Cotation Assistée en Continu, é o índice da Bolsa de Valores da França que reúne as 40 maiores empresas do país. Já o DAX30, abreviação de Deutscher AktienindeX, é o índice Alemão que reúne as 30 empresas com melhor performance financeira do país.

Propõe-se então o ajuste da função cópula considerando os índices francês e alemão para analisar se existe dependência entre as séries. 
Foram considerados índices CAC40 e DAX30 obtidos de 31/12/1993 a 08/08/2000, com um total de 1.723 observações.

Na Figura 6.1 são apresentados os gráficos dos log-retornos das séries.
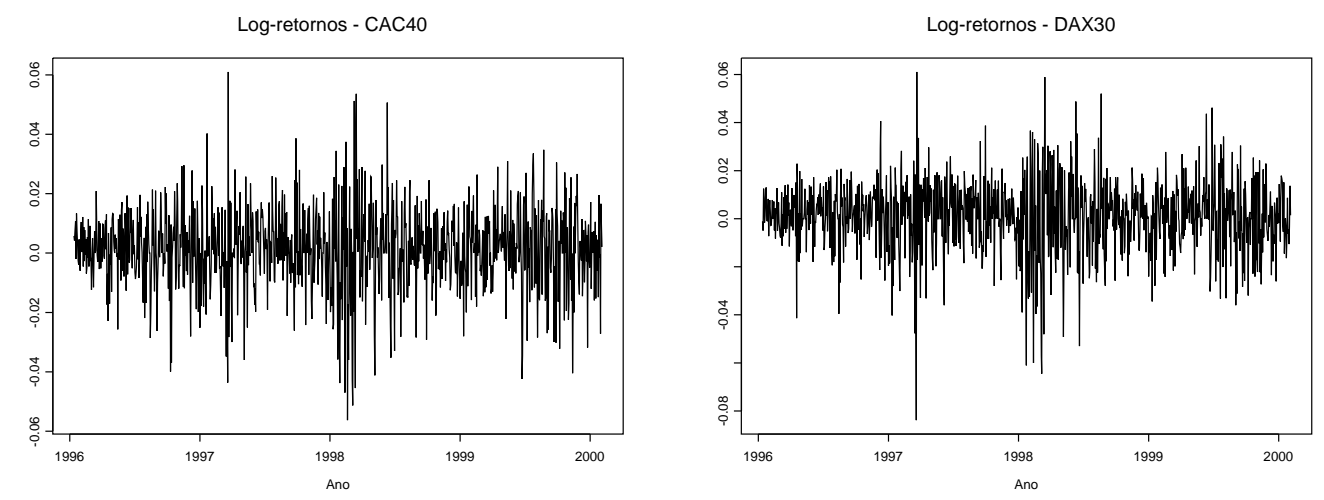

Figura 6.1: Gráficos dos log-retornos das séries.

O gráfico de dispersão entre as séries CAC40 e DAX30 é apresentado na Figura 6.2. O gráfico indica que aparentemente há correlação entre as séries. O coeficiente de correlação calculado entre as séries é de 0,6702, indicando uma correlação moderada.

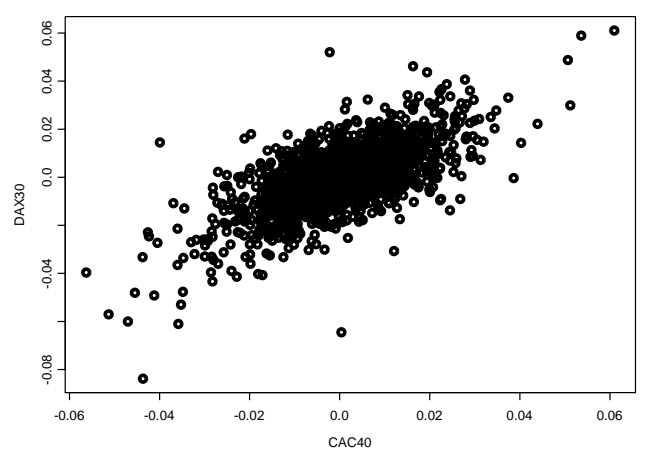

Figura 6.2: Gráfico de dispersão entre as séries CAC40 e DAX30.

Para a aplicação, foram utilizadas as últimas 1.024 observações. Considerando a ondaleta Daubechie "D2", foi feito o ajuste da cópula para as séries CAC40-DAX30, 
com $l^{*}=5$ utilizando somente a função escala. Os gráficos da cópula ajustada e das curvas de nível são apresentados na Figura 6.3.
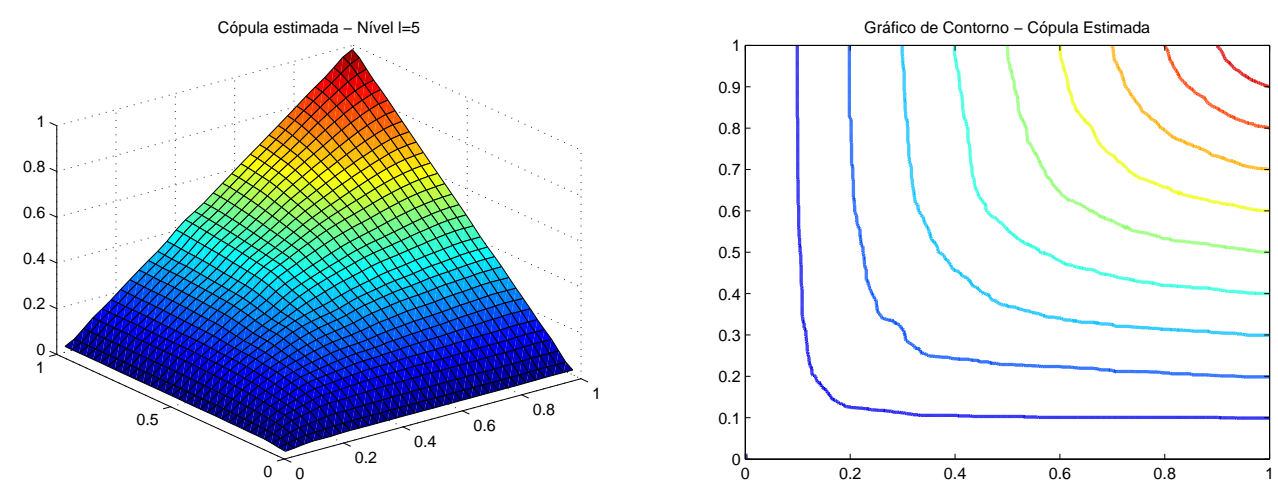

Figura 6.3: Gráficos da cópula ajustada via ondaletas e das curvas de nível

Visando analisar o comportamento das cópulas ajustadas via ondaletas, as mesmas serão comparadas com algumas cópulas paramétricas ajustadas.

No caso do ajuste de cópulas paramétricas para séries temporais, como pode ser visto em Morettin (2011), a amostra não é composta por pares independentes. Uma sugestão para contornar este problema é ajustar um modelo ARMA-GARCH a cada série, obter os respectivos resíduos padronizados, e então ajustar uma cópula paramétrica a esses resíduos.

De acordo com análises preliminares, foi ajustado um modelo $\operatorname{AR}(7)-\mathrm{GARCH}(1,1)$ para a série CAC40, e um modelo $\operatorname{AR}(6)-G A R C H(1,1)$ para a série DAX30. Como pode ser visto no Apêndice B, ambas considerando que os erros seguem uma distribuição t-Student. Para o ajuste dos modelos, foi utilizado o software S-Plus 8.0.

Aos resíduos padronizados, foram ajustadas as cópulas Normal, t-Student, Gumbel, Frank e Clayton. Foi utilizado o método de máxima verossimilhança. Os ajustes também podem ser vistos no Apêndice B, feito por meio do software R i386 3.0.2. As estimativas dos parâmetros são apresentadas na Tabela 6.1.

Note que, no caso do ajuste considerando cópulas paramétricas, para analisar 
qual o ajuste seria o mais adequado, o valor da máxima verossimilhança pode ser um indicativo.

De acordo com os resultados, a cópula ajustada que apresentou maior valor da verossimilhança foi a cópula t-Student, indicando que para esse conjunto de dados, a cópula t-Student é a mais adequada.
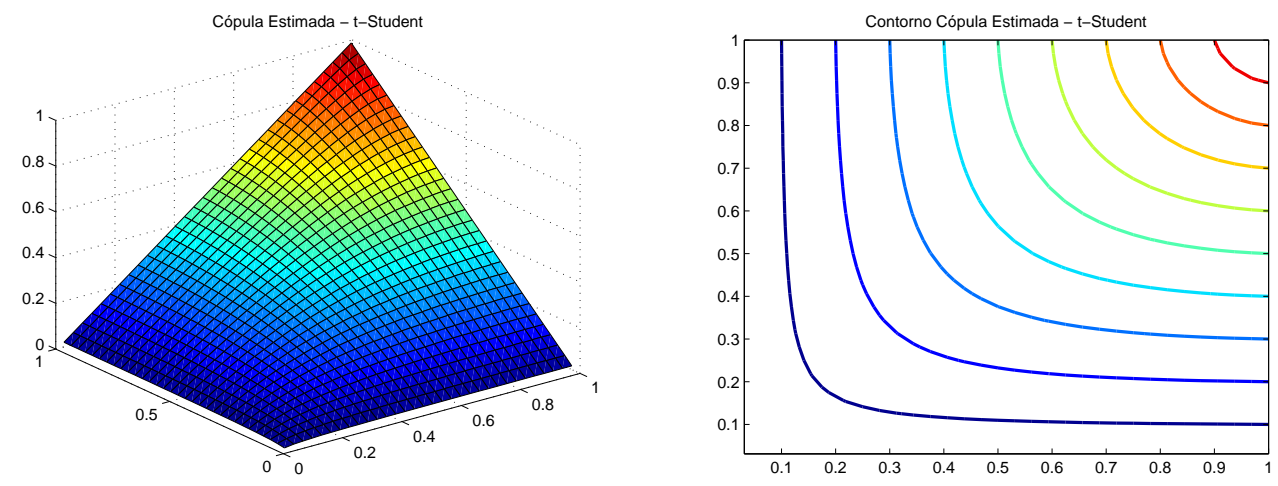

Figura 6.4: Gráficos da cópula ajustada e das curvas de nível - t-Student

Na Figura 6.4 são apresentados os gráficos da cópula t-Student estimada e das curvas de nível.

Os gráficos das curvas de nível apresentados para os ajustes de cópulas, tanto no caso não paramétrico utilizando ondaletas, como no caso paramétrico, apresentados nas Figuras 6.3 e 6.4, indicam que as séries tem uma estrutura de dependência moderada e além disso que os ajustes apresentam valores próximos, evidenciando que o estimador via ondaletas, no caso não paramétrico para dados dependentes controla o fato dos dados não serem independentes, o que é feito através de ajustes ARMAGARCH nos modelos paramétricos.

Porém, dizer que os valores são aparentemente próximos utilizando somente os gráficos das curvas de nível pode ser algo superficial, sendo assim, visando analisar qual o comportamento da cópula ajustada via ondaletas, em relação as cópulas paramétricas ajustadas, foram calculados os valores do Erro e Erro Relativo(ER). 


\begin{tabular}{c||c||c|c||c|c||c|c||c|c}
\multicolumn{2}{c||}{ Paramétrico } & \multicolumn{2}{c||}{ Ondaleta "D2" } & \multicolumn{2}{c||}{ Ondaleta "D4" } & \multicolumn{2}{c||}{ Ondaleta "D6" } & \multicolumn{2}{c}{ Ondaleta - "D8" } \\
\hline Cópula & Estimativa & Erro & ER & Erro & ER & Erro & ER & Erro & ER \\
\hline \hline Normal & 0,67391 & 0,4672 & 3,2476 & 0,4673 & 3,2484 & 0,4675 & 3,2494 & 0,4675 & 3,2498 \\
\hline Student & $0,6857(5,43)$ & $\mathbf{0 , 3 5 4 6}$ & $\mathbf{2 , 4 5 8 2}$ & 0,3548 & 2,4590 & 0,3549 & 2,4601 & 0,3550 & 2,4605 \\
\hline Gumbel & 1,8013 & 1,5042 & 10,4658 & 1,5043 & 10,4666 & 1,5045 & 10,4677 & 1,5046 & 10,4681 \\
\hline Clayton & 1,4081 & 1,7169 & 12,6474 & 1,7170 & 12,6483 & 1,7172 & 12,6494 & 1,7173 & 12,6498 \\
\hline Frank & 5,5571 & 0,8271 & 5,6803 & 0,8273 & 5,6811 & 0,8274 & 5,6822 & 0,8275 & 5,6826 \\
\hline
\end{tabular}

Tabela 6.1: Erro e ER entre as cópulas estimadas.

A Tabela 6.1 apresenta os valores do Erro e do ER, múltiplos de $10^{-4}$, entre as cópulas paramétricas ajustadas e as cópulas estimadas por meio de ondaletas, considerando as ondaletas Daubechie "D2", "D4", "D6" e "D8". Na tabela também são apresentadas as estimativas dos parâmetros das cópulas ajustadas, em que para a cópula Normal, o parâmetro estimado é o coeficiente de correlação linear, para a cópula t-Student são o coeficiente de correlação linear e os graus de liberdade e para as cópulas Gumbel, Clayton e Frank, denominadas cópulas arquimedianas, o parâmetro estimado é o que mede o grau de dependência entre as séries, utilizado na função geradora das cópulas arquimedianas. Para mais detalhes, veja Nelsen (2005).

Analisando a Tabela 6.1, os menores valores para os erros foram obtidos entre as cópulas t-Student ajustada (para o modelo paramétrico) e a cópula via ondaletas, considerando a Daubechie "D2". Note que os valores não se alteram muito quando foram consideradas outras ondaletas, o que indica que neste caso, o ajuste não paramétrico é pouco alterado considerando outros tipos de ondaletas.

Os valores da tabela são um indicativo de que a cópula estimada via ondaletas, apresenta valores próximos aos da cópula t-Student ajustada, porém sem suposição alguma sobre o comportamento dos dados, e também sem a necessidade de ajustes de modelos ARMA-GARCH, além de utilizar somente a função escala, não utilizando funções de detalhes, diminuindo o número de coeficientes estimados.

Dados os valores das cópulas estimadas via ondaletas, é possível verificar de que forma as séries podem estar associadas. Uma maneira de analisar essa possível as- 
sociação é estimar os parâmetros de dependência nas caudas superior e inferior das séries, utilizando cópulas.

Para as medidas de dependência nas caudas, existem os estimadores paramétricos, que no caso da cópula normal e t-Student são associados ao coeficiente de correlação linear. Já para as cópulas arquimedianas, nem todas permitem o cálculo dessas medidas, mas para as que permitem, o cálculo dessas medidas está associado ao parâmetro que mede o grau de dependência.

Tendo como objetivo estimar as medidas de dependência por meio de cópula, sabendo que a cópula foi estimada via ondaletas, seria mais adequado utilizar um estimador não paramétrico para as medidas.

Assim, serão propostos os estimadores dados por Schmidt and Stadtmüller (2006), definidos por

$$
\hat{\lambda}_{L}\left(\frac{i}{n}\right)=\frac{\hat{C}\left(\frac{i}{n} \frac{i}{n}\right)}{\frac{i}{n}}
$$

e

$$
\hat{\lambda}_{U}\left(\frac{i}{n}\right)=\frac{1-\frac{2 i}{n}+\hat{C}\left(\frac{i}{n} \frac{i}{n}\right)}{1-\frac{i}{n}}
$$

em que $\hat{\lambda}_{L}$ é o estimador para a medida de dependência na cauda inferior, $\hat{\lambda}_{U}$ é o estimador para a medida na cauda superior, $i \in\{1,2, \ldots, n-1\}$ e $n$ é tamanho amostral. Neste caso, será utilizado o estimador proposto via ondaletas ao invés da cópula empírica $\hat{C}\left(\frac{i}{n} \frac{i}{n}\right)$.

Na Figura 6.5 são apresentados os gráficos das estimativas para as medidas de dependência nas caudas inferior e superior, para as séries CAC40 e DAX30, calculadas para os pares de pontos $\left(\frac{i}{n}, \frac{i}{n}\right)$ próximos as caudas.

Os gráficos indicam que existe dependência nos quantis inferiores, o que também ocorre nos quantis superiores, evidenciando que há dependência entre as séries. 

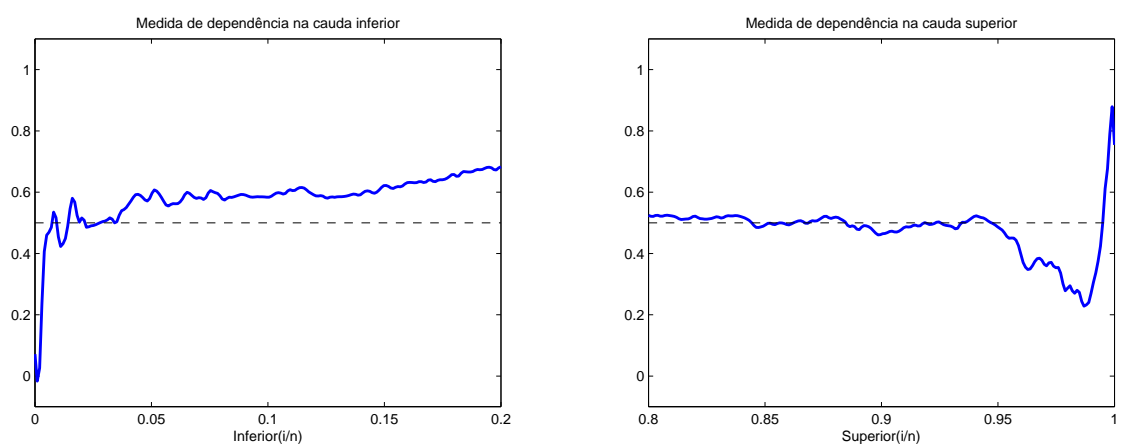

Figura 6.5: Gráfico das estimativas para as medidas de dependência nas caudas

\subsubsection{Séries Ibovespa - IPC}

Nesta seção serão analisadas as séries Ibovespa e IPC.

Ibovespa, abreviação de índice Bovespa é o mais importante indicador do desempenho médio das cotações das ações negociadas na BM\&FBOVESPA (Bolsa de Mercadorias e Futuros de São Paulo). IPC, abreviação de Índice de Precios y Cotizaciones é o principal índice da Bolsa Valores do México, formado pelas 35 empresas com maior liquidez no mercado.

Os log-retornos das séries são apresentados na Figura 6.6.
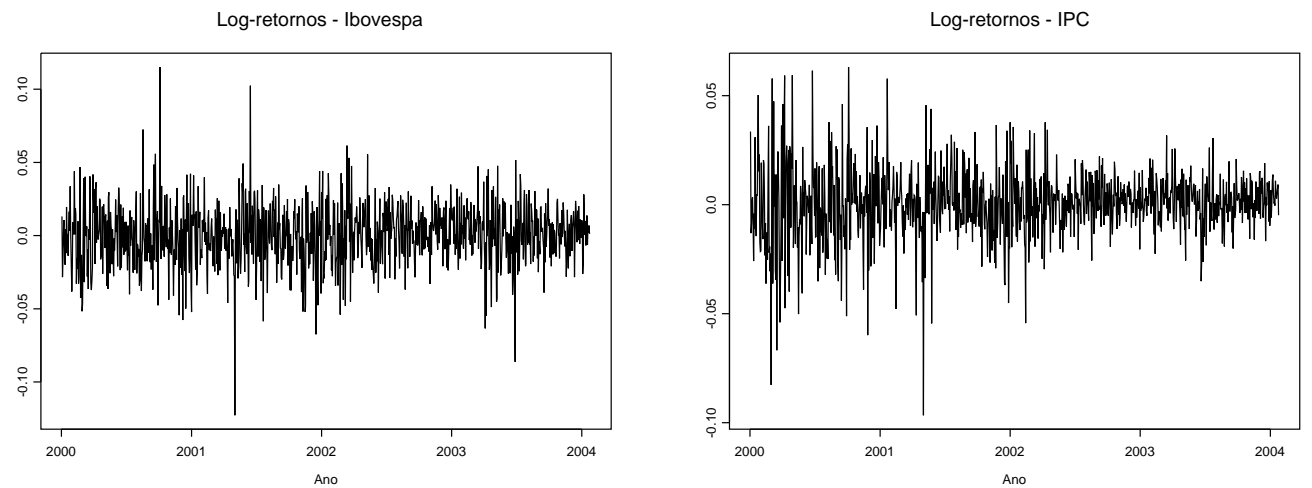

Figura 6.6: Gráficos dos log-retornos das séries. 
Os índices obtidos são de 04/09/1995 a 30/12/2004, em um total de 1.981 observações para cada uma das duas séries.

A Figura 6.7 apresenta o gráfico de dispersão entre as séries Ibovespa e IPC, com coeficiente de correlação 0,5516, também indicando correlação moderada entre as séries.

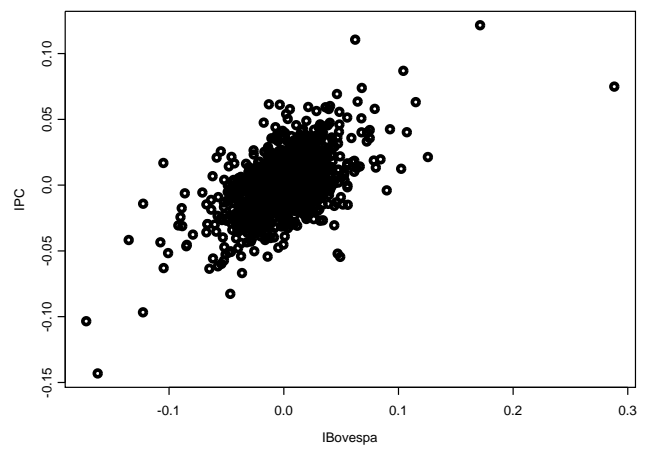

Figura 6.7: Gráfico de dispersão entre as séries Ibovespa e IPC.

Utilizando somente a função escala, foi feito o ajuste da cópula para as últimas 1.024 observações das séries Ibovespa-IPC, com $l^{*}=5$, considerando também a ondaleta Daubechie "D2". Os gráficos da cópula ajustada e das curvas de nível são apresentados na Figura 6.8.

Como no caso anterior, também foram ajustadas algumas cópulas paramétricas para fins de comparação com as cópulas ajustadas via ondaletas.

Antes do ajuste das cópulas paramétricas, uma vez que os dados não são independentes, serão ajustados os modelos ARMA-GARCH para ambas as séries, e então obtidos os resíduos padronizados necessários para o ajuste de cópulas paramétricas.

Para a série Ibovespa, não foi necessário fazer ajustes de modelos ARMA-GARCH. Já para a série IPC foi ajustado um modelo $\operatorname{GARCH}(1,1)$. Aos resíduos padronizados, foram ajustadas as cópulas paramétricas como pode ser visto no Apêndice B.

O ajuste que apresentou o maior valor para a verossimilhança foi para a cópula 

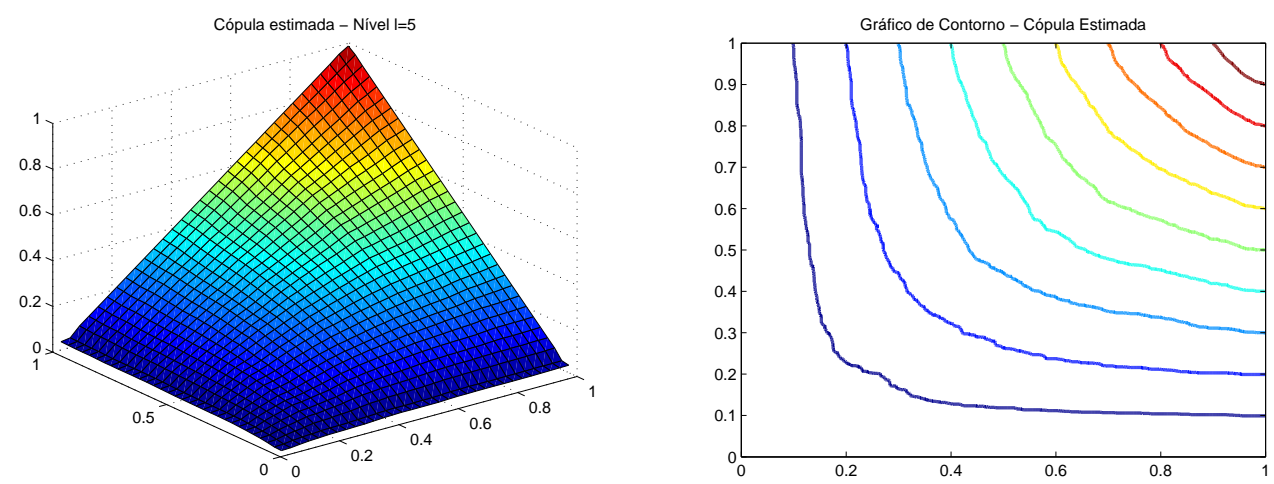

Figura 6.8: Gráficos da cópula estimada e das curvas de nível para as séries Ibovespa e IPC - via ondaletas.

t-Student, indicando ser o ajuste paramétrico mais adequado. A Figura 6.9 apresenta os gráficos da cópula t-Student estimada para as séries Ibovespa e IPC.
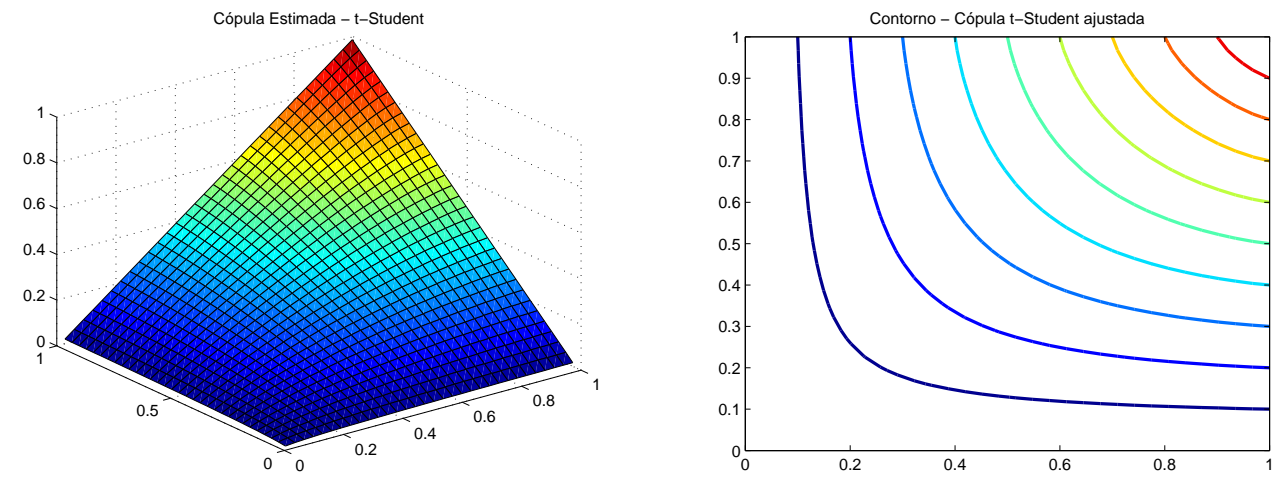

Figura 6.9: Gráficos da cópula estimada t-Student e das curvas de nível para séries Ibovespa IPC.

Os gráficos das curvas de nível, apresentados nas Figuras 6.8 e 6.9 aparentemente indicam que as cópulas ajustadas apresentam valores próximos, porém como dito anteriormente, essa afirmação é feita somente pela observação dos gráficos.

Para analisar, não só graficamente, o comportamento do ajuste não paramétrico em relação ao paramétrico, são calculados os valores do Erro e do ER entre as cópulas 
paramétricas estimadas e as cópulas estimadas considerando ondaletas Daubechies. Os valores, todos múltiplos de $10^{-4}$, são apresentados na Tabela 6.2, que apresenta também as estimativas dos ajustes das cópulas paramétricas.

\begin{tabular}{c||c||c|c||c|c||c|c||c|c}
\multicolumn{2}{c||}{ Paramétrico } & \multicolumn{2}{c||}{ Ondaleta "D2" } & \multicolumn{2}{c||}{ Ondaleta "D4" } & \multicolumn{2}{c||}{ Ondaleta "D6" } & \multicolumn{3}{c}{ Ondaleta "D8" } \\
\hline Cópula & Estimativa & Erro & ER & Erro & ER & Erro & ER & Erro & ER \\
\hline \hline Normal & 0,4356 & 0,3418 & 2,6173 & 0,3418 & 2,6175 & 0,3418 & 2,6176 & 0,3418 & 2,6177 \\
\hline Student & $0,4439(7,45)$ & $\mathbf{0 , 2 9 3 3}$ & $\mathbf{2 , 2 4 0 1}$ & 0,2934 & 2,2403 & 0,2934 & 2,2403 & 0,2934 & 2,2405 \\
\hline Gumbel & 1,3559 & 1,1778 & 9,0066 & 1,1778 & 9,0069 & 1,1778 & 9,0070 & 1,1778 & 9,0071 \\
\hline Clayton & 0,6529 & 0,8760 & 7,0072 & 0,8760 & 7,0075 & 0,8760 & 7,0076 & 0,8760 & 7,0077 \\
\hline Frank & 2,8910 & 0,3743 & 2,8398 & 0,3743 & 2,8400 & 0,3743 & 2,8401 & 0,3744 & 2,8402 \\
\hline
\end{tabular}

Tabela 6.2: Erro e ER entre as cópulas estimadas para as séries Ibovespa - IPC.

Como no caso anterior, os erros obtidos apresentaram menores valores entre as cópulas t-Student (para o modelo paramétrico) e a cópula utilizando ondaleta Daubechie "D2".

Também foram ajustadas as medidas de dependência nas caudas, como pode ser visto na Figura 6.10.
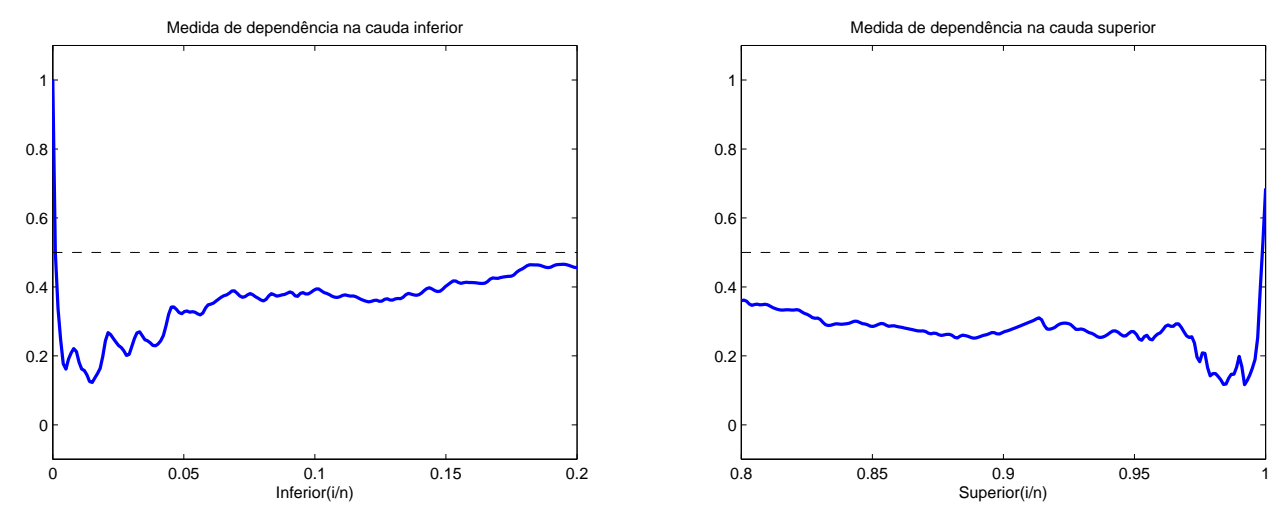

Figura 6.10: Gráfico das estimativas para as medidas de dependência nas caudas para as séries Ibovespa e IPC.

Os gráficos apresentados na Figura 6.10 indicam que há dependência fraca nos quantis inferiores e superiores para as séries. 


\subsubsection{Séries S\&P500 - DJIA}

S\&P550, abreviação de Standard \& Poor 500, é um índice americano composto por ativos de 500 grandes empresas. Já o Dow Jones Industrial Average, abreviado por DJIA, é o segundo maior índice dos Estados Unidos baseado na cotação das ações das 30 maiores e mais importantes empresas americanas.

Os índices obtidos são de 31/12/1993 a 08/08/2000, totalizando 1.723 observações. Os log-retornos das séries são apresentados na Figura 6.11.
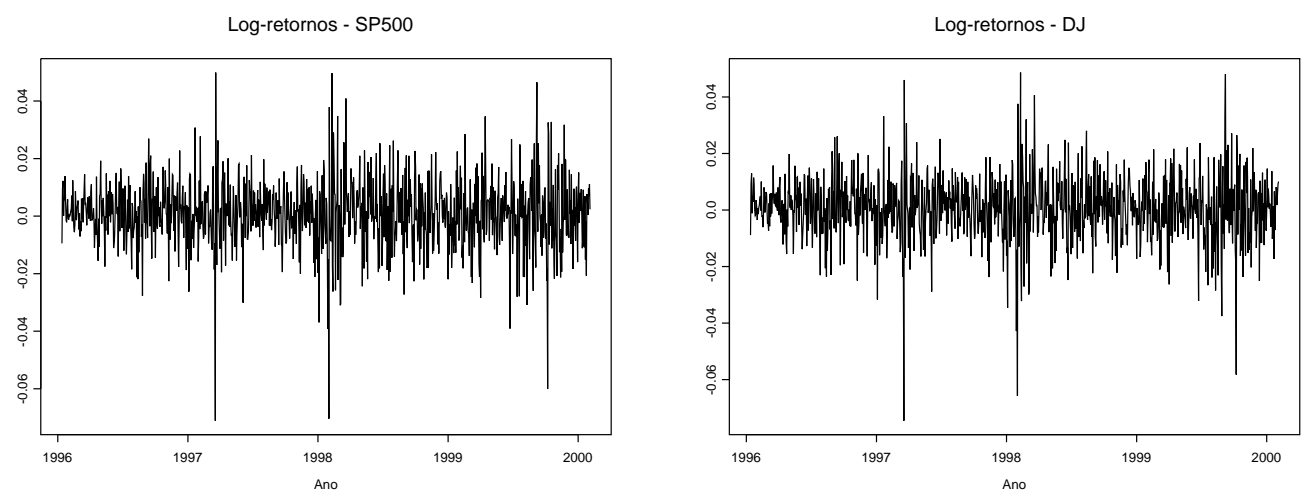

Figura 6.11: Gráficos dos log-retornos das séries.

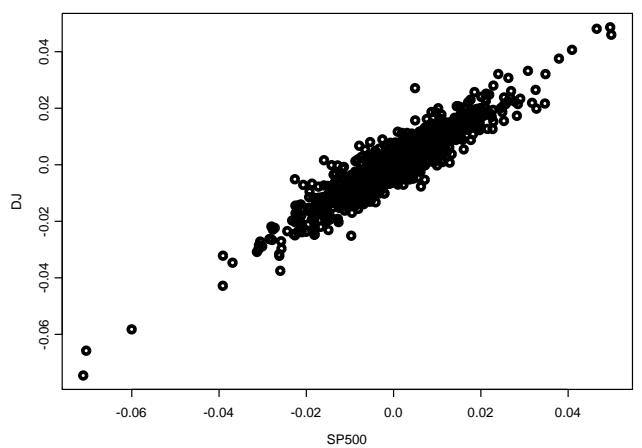

Figura 6.12: Gráfico de dispersão entre as séries S\&P500 e DJIA. 
A Figura 6.12 apresenta o gráfico de dispersão entre as séries. O coeficiente de correlação calculado entre as séries é de 0,9309, indicando alta correlação.

Assim como nos casos anteriores, deseja-se avaliar de que forma as duas séries podem estar correlacionadas. Para isso, propõe-se o ajuste de cópulas por meio de ondaletas, considerando as últimas 1.024 observações, utilizando a ondaleta Daubechie "D2", $\operatorname{com} l^{*}=5$.

Os gráficos com o ajuste da cópula por meio de ondaletas são apresentados na Figura 6.13.
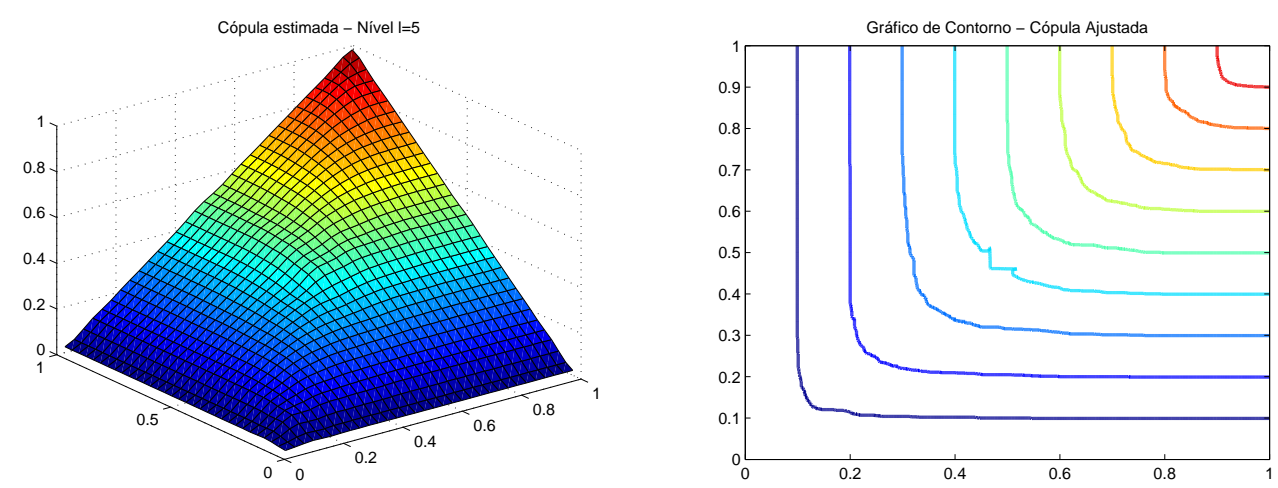

Figura 6.13: Gráficos da cópula ajustada e das curvas de nível para as séries S\&P500 e DJIA via ondaletas.

Nota-se pelo gráfico das curvas de nível, à direita da Figura 6.13, que há dependência forte entre as séries quando comparado ao gráfico de curvas de nível de séries com co-monotonicidade.

A cópula estimada via ondaletas também foi comparada ao ajuste de algumas cópulas paramétricas.

Para o ajuste de cópulas paramétricas, foi ajustado o modelo $\operatorname{AR}(7)-\mathrm{GARCH}(1,1)$ para as séries S\&P500 e DJIA, e aos resíduos padronizados foram ajustadas as cópulas Normal, t-Student, Gumbel, Clayton e Frank. Os ajustes também são apresentados no Apêndice B.

Como nos casos anteriores, o ajuste que apresentou maior valor para a verossimi- 
lhança foi para a cópula t-Student. Na Figura 6.14 são apresentados os gráficos da cópula t-Student estimada e das curvas de nível.
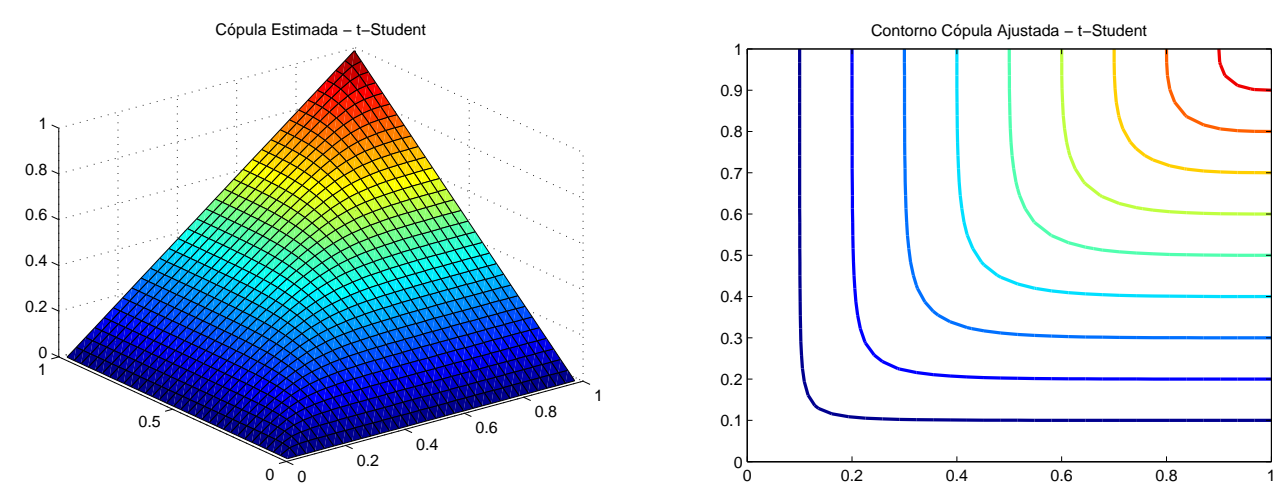

Figura 6.14: Gráficos da cópula t-Student ajustada e das curvas de nível para séries S\&P500 DJIA.

Assim como nos casos anteriores, os gráficos das curvas de nível para as cópulas ajustadas no caso paramétrico e via ondaletas, apresentados nas Figuras 6.13 e 6.14 indicam que os ajustes apresentam valores próximos, além de mostrar que existe uma alta correlação entre as séries. Também foram calculados os Erros e ER para analisar os ajustes paramétrico e não paramétrico.

\begin{tabular}{c||c||c|c||c|c||c|c||c|c}
\multicolumn{2}{c||}{ Paramétrico } & \multicolumn{2}{c||}{ Ondaleta "D2" } & \multicolumn{2}{c||}{ Ondaleta "D4" } & \multicolumn{2}{c||}{ Ondaleta "D6" } & \multicolumn{2}{c}{ Ondaleta "D8" } \\
\hline Cópula & Estimativa & Erro & ER & Erro & ER & Erro & ER & Erro & ER \\
\hline \hline Normal & 0,9214 & 0,1155 & 0,7209 & 0,1175 & 0,7335 & 0,1163 & 0,7259 & 0,1158 & 0,7229 \\
\hline Student & $0,9218(5,19)$ & $\mathbf{0 , 1 0 0 0}$ & $\mathbf{0 , 6 2 5 1}$ & 0,1020 & 0,6378 & 0,1008 & 0,6301 & 0,1003 & 0,6271 \\
\hline Gumbel & 3,6690 & 0,2143 & 1,3400 & 0,2163 & 1,3527 & 0,2151 & 1,3450 & 0,2146 & 1,3420 \\
\hline Clayton & 3,9255 & 1,8597 & 12,2000 & 1,8618 & 12,2133 & 1,8605 & 12,2053 & 1,8601 & 12,2021 \\
\hline Frank & 13,6540 & 0,3488 & 2,1744 & 0,3508 & 2,1870 & 0,3496 & 2,1794 & 0,3491 & 2,1764 \\
\hline
\end{tabular}

Tabela 6.3: Erro e ER entre as cópulas ajustadas para as séries S\&P500 - DJIA.

Na Tabela 6.3 são apresentados os valores das estimativas obtidas para as cópulas paramétricas ajustadas, além dos valores do Erro e ER, múltiplos de $10^{-4}$, entre as cópulas paramétricas ajustadas e a cópula estimada via ondaletas. Os menores valores 
para o Erro e o ER foram obtidos entre as cópulas ajustadas utilizando ondaleta Daubechie "D2" e a cópula t-Student.

Na Figura 6.15 são apresentados os gráficos das estimativas para as medidas de dependência nas caudas para as séries S\&P500 e DJIA.
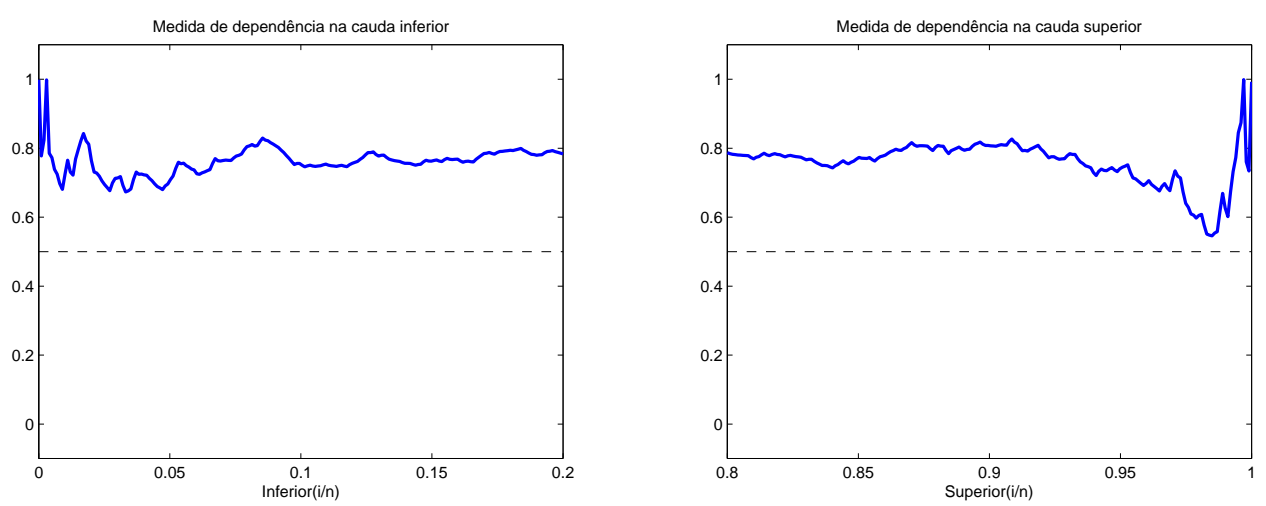

Figura 6.15: Gráfico das estimativas para as medidas de dependência nas caudas para as séries S\&P500 e DJIA.

Assim como indicado pelos gráficos das curvas de nível, existe uma alta dependência entre as séries, como pode ser visto nos gráficos das medidas de dependência calculadas para os quantis. Tanto para os altos quantis quanto para os baixo quantis, os valores são próximos a um.

No período analisado, aparentemente há uma relação co-monotônica entre as séries S\&P500 e DJIA.

\subsection{Caso iid}

Para a aplicação no caso iid, serão consideradas as séries de índices anuais de desempenho das vendas de grandes empresas privadas e estatais (exceto banco e seguradoras) no ano de 2006, de acordo com o critério da revista Exame, totalizando 1018 empresas, utilizadas em Latif e Morettin (2010). 
Para analisar se existe estrutura de dependência entre os índices, utilizando o estimador via ondaletas, serão considerados os Índices de vendas (US \$ milhões), margem sobre as vendas (\%) e lucro líquido (US \$ milhões) das 864 empresas que continham estas informações.

\subsubsection{Séries Lucro líquido - Margem de vendas}

Inicialmente, foram consideradas as variáveis lucro líquido e margem de venda. Os postos padronizados para ambas as séries apresentaram um coeficiente de correlação de 0,8455 , indicando uma alta correlação, como é possível ver pelo gráfico de dispersão, apresentando na Figura 6.16. O comportamento do gráfico sugere que, considerando o ajuste de cópulas paramétricas, a cópula Clayton seja a mais adequada.

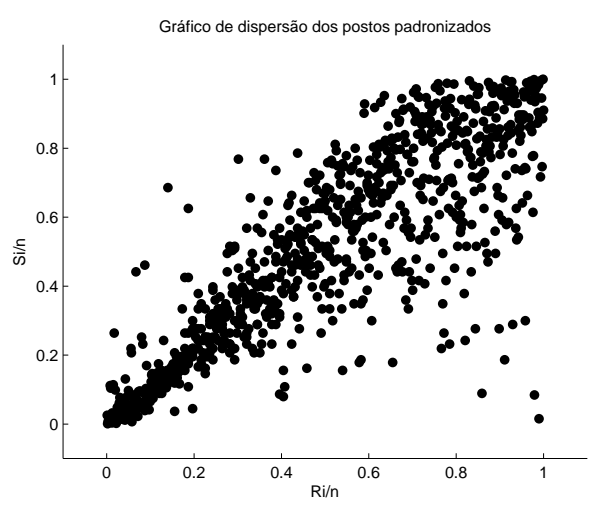

Figura 6.16: Gráfico de dispersão entre os postos padronizados das séries Lucro líquido e Margem de vendas.

Na Figura 6.17 são apresentados os gráficos da cópula ajustada via ondaleta aos postos padronizados, utilizando ondaleta Daubechie "D2", para um nível $l^{*}=5$.

Para fins de comparação, foram também ajustadas cópulas paramétricas aos postos padronizados das séries. Os ajustes podem ser vistos no Apêndice B.

A cópula paramétrica ajustada que apresentou o maior valor para a máxima verossimilhança foi a cópula Clayton, indicando que este seria o ajuste mais indicado, 

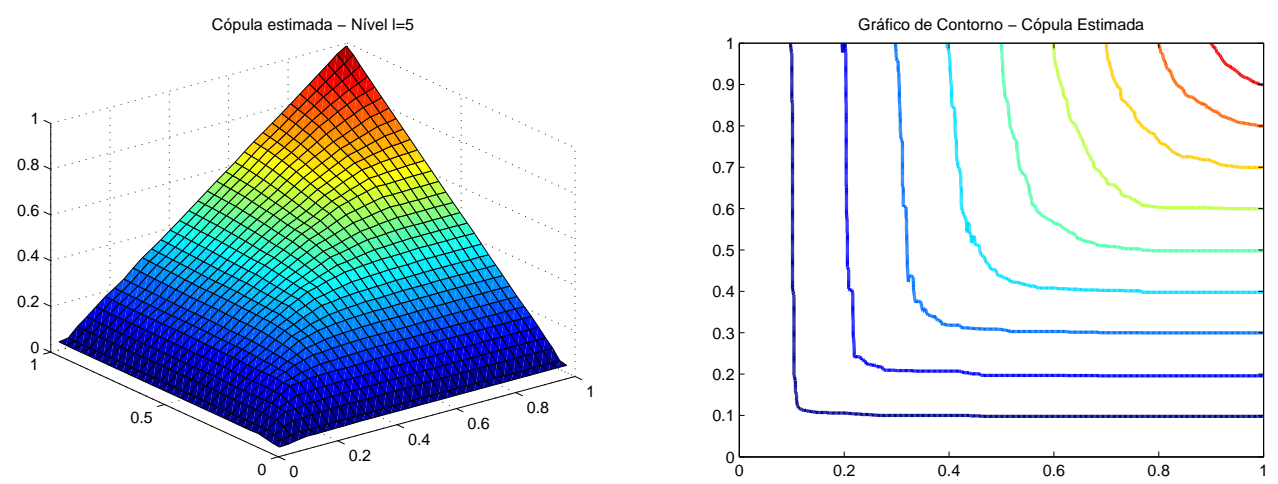

Figura 6.17: Gráficos da cópula ajustada via ondaletas e das curvas de nível

como comentado anteriormente.

A Figura 6.18 apresenta o gráfico da cópula Clayton ajustada e das curvas de nível.
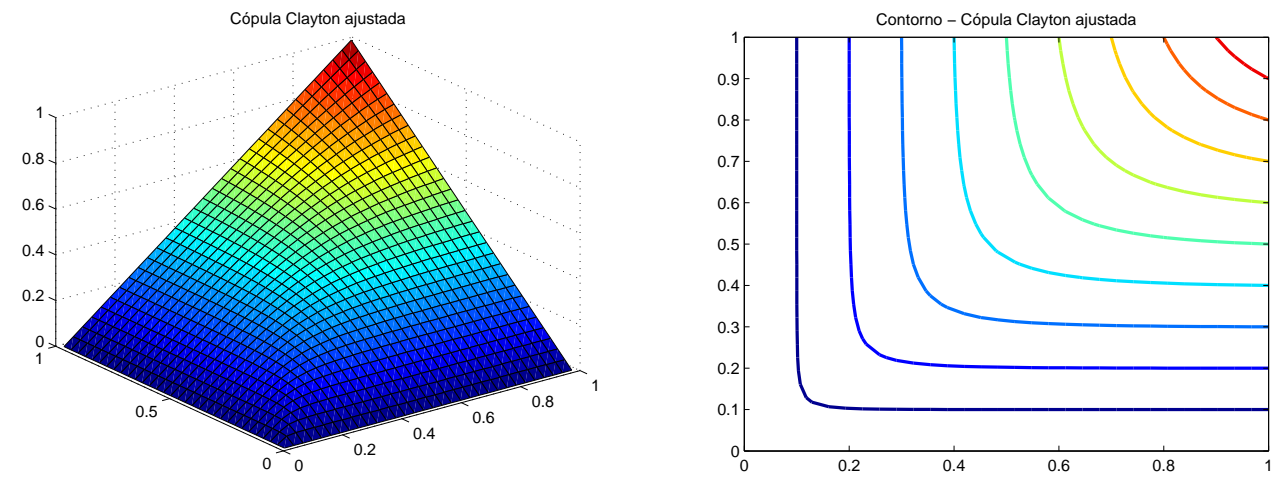

Figura 6.18: Gráfico das estimativas para as medidas de dependência nas caudas para as séries lucro líquido e margem de venda.

De acordo com as Figuras 6.17 e 6.18, os gráficos das curvas de nível, além de indicarem que existe uma alta correlação entre as séries, aparentemente indicam também que os ajustes apresentam valores próximos. Para analisar quão próximos estão, calculou-se os valores do Erro e ER, como feito no caso dependente, considerando 
várias ondaletas Daubechies.

\begin{tabular}{c||c||c|c||c|c||c|c||c|c}
\multicolumn{2}{c||}{ Paramétrico } & \multicolumn{2}{c||}{ Ondaleta "D2" } & \multicolumn{2}{c||}{ Ondaleta "D4" } & \multicolumn{2}{c||}{ Ondaleta "D6" } & \multicolumn{2}{c}{ Ondaleta "D8" } \\
\hline Cópula & Estimativa & Erro & ER & Erro & ER & Erro & ER & Erro & ER \\
\hline \hline Normal & 0,8337 & 1,3194 & 8,5800 & 1,3195 & 8,5807 & 1,3200 & 8,5838 & 1,3203 & 8,5857 \\
\hline Student & $0,8587(3,35)$ & 1,0474 & 6,7614 & 1,0475 & 6,7620 & 1,0480 & 6,7652 & 1,0483 & 6,7670 \\
\hline Gumbel & 2,4695 & 2,8513 & 18,6410 & 2,8514 & 18,6416 & 2,8519 & 18,6448 & 2,8522 & 18,6467 \\
\hline Clayton & 3,5664 & $\mathbf{0 , 5 6 3 6}$ & $\mathbf{3 , 7 3 5 8}$ & 0,5637 & 3,7364 & 0,5642 & 3,7397 & 0,5645 & 3,7416 \\
\hline Frank & 10,3942 & 0,9937 & 6,3265 & 0,9938 & 6,3271 & 0,9943 & 6,3303 & 0,9946 & 6,3321 \\
\hline
\end{tabular}

Tabela 6.4: Erro e ER entre as cópulas estimadas para as séries lucro líquido - margem de venda.

As estimativas para os ajustes de cópulas paramétricas são apresentadas na Tabela 6.4, assim como os valores do Erro e ER calculados, múltiplos de $10^{-4}$. Os menores valores obtidos para os erros foram entre a cópula Clayton ajustada e a cópula ajustada utilizando ondaleta Daubechie "D2".

Também são calculadas as medidas de dependência nas caudas inferiores e superiores, como podem ser vistas pelos gráficos apresentados na Figura 6.19 a seguir.
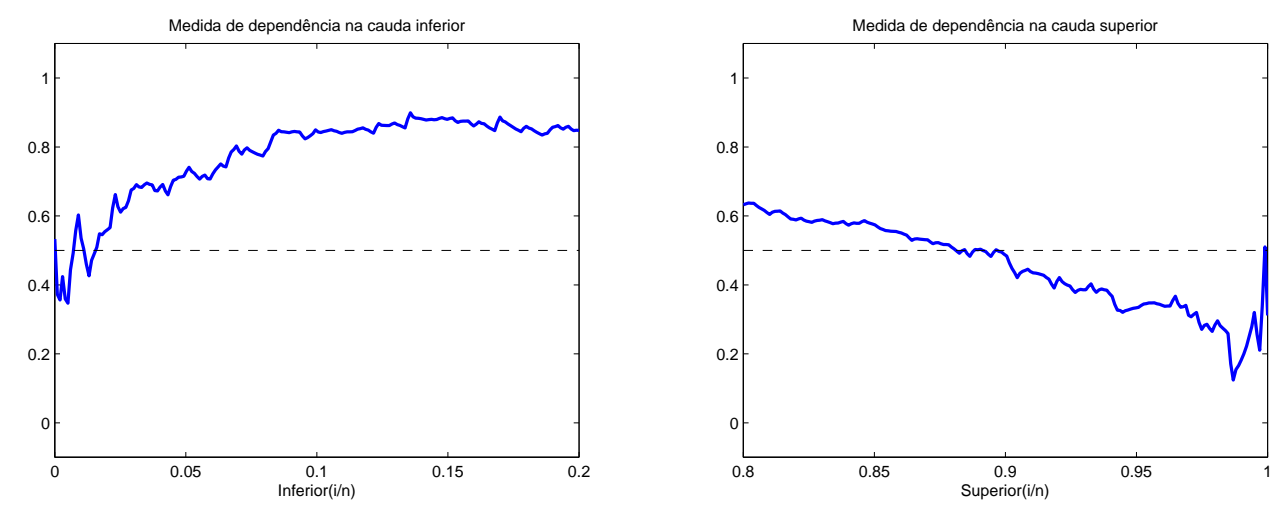

Figura 6.19: Gráfico das estimativas para as medidas de dependência nas caudas para as séries lucro líquido e margem de venda.

Os gráficos das medidas de dependência indicam que nas caudas inferiores existe mais dependência que nas caudas superiores, indicando que uma série é relacionada 
com a outra para os menores quantis.

\subsection{Comentários}

Neste capítulo foram ajustadas cópulas utilizando a metodologia proposta para os casos dependente e independente. Verificou-se que ao utilizar o estimador via ondaletas, não é necessário fazer suposições ou afirmações preliminares sobre o comportamento dos dados.

Foi possível verificar também que em todos os casos, o ajuste via ondaletas apresentou resultados próximos aos obtidos no caso paramétrico indicado como o mais adequado, evidenciando que o método contorna o problema dos pares não serem independentes, que ocorre no caso de ajuste de cópulas para séries temporais. 


\section{Capítulo 7}

\section{Conclusões}

A estimação de densidades de cópulas via ondaletas tem sido proposta recentemente por inúmeros autores, que visam a estimação de forma precisa. Porém, a estimação direta da função cópula surge como uma proposta de obter uma aproximação mais precisa, sem a necessidade do ajuste das funções de distribuição marginais.

Neste trabalho foi proposto um estimador para a função cópula, a partir da expansão da função em bases de ondaletas, tanto para o caso iid como para o caso de séries temporais.

Foram calculadas as taxas de convergência, utilizando uma função de risco em ambos os casos. Para o caso de séries temporais, foram feitas algumas suposições sobre a estrutura de dependência do processo, neste caso a condição $\alpha$-mixing.

Foram feitos estudos de simulação para ambos os casos, considerando variáveis correlacionadas e não correlacionadas, com o intuito de analisar o desempenho do estimador perante vários cenários.

Para variáveis não correlacionadas, nos casos de amostras dependentes e independentes foram obtidos resultados satisfatórios, o que pode ser verificado no caso dependente quando os resultados foram comparados a outros métodos propostos na literatura.

No caso de variáveis aleatórias correlacionadas, em ambos os casos os resultados 
apresentaram maiores valores para o Viés, o que levou a análise do estimador utilizando algumas funções de detalhes selecionadas pelo método de threshold. Com o uso das funções de detalhes, as simulações apresentaram menores valores para o Viés, evidenciando a importância do uso de funções de detalhes no caso dependente.

Visando analisar qual o comportamento do estimador para diferentes tamanhos amostrais, foram feitas algumas simulações adicionais propondo o uso do método sugerido em Autin et al. (2010). Os resultados indicaram que o desvio padrão tende a diminuir para maiores valores amostrais.

As aplicações foram feitas considerando várias séries importantes no cenário econômico mundial.

Foi ajustada a cópula para as séries de índices CAC40, francesa e a alemã DAX30, que apresentaram uma estrutura de dependência moderada.

O ajuste para as séries Ibovespa do Brasil e IPC do México indica que há fraca dependência entre as séries.

Já para os índices S\&P500 e DJIA o ajuste indicou que há forte dependência, provável pelo fato de ambas serem americanas.

Para o caso independente, o ajuste foi feito para as séries Lucro líquido e Margem de vendas medidos para 864 empresas privadas e estatais de acordo com a revista Exame. Pelos gráficos de curvas de nível a cópula ajustada indica que há alta dependência, como foi verificado pelos gráficos de medidas de dependência ajustadas nas caudas.

Em todos os casos também foram feitos ajustes de cópulas paramétricas com o intuito de compará-las ao método proposto. Os menores valores obtidos para o Erro e ER foram entre as cópulas ajustadas via ondaletas e as ajustadas considerando o melhor modelo paramétrico, indicando que a estimação via ondaletas está sempre mais próxima a estimação do melhor modelo paramétrico. O estimador proposto, entretando, utiliza somente a função escala, sem a necessidade de ajustes preliminares de modelos ARMA-GARCH, além de não fazer suposição alguma sobre a distribuição dos dados. 


\section{Considerações Finais}

De forma geral, após os estudos de simulação, foi verificado que o estimador, no caso dependente apresentou melhores resultados quando utilizadas as funções de detalhes, selecionadas por threshold. Além disso, as taxas de convergência indicam a necessidade da escolha de um nível $l^{*}$ considerado ótimo, que depende do grau de regularidade da função a ser estimada, que muitas vezes é deconhecido. Outro fato interessante também, é que no contexto de séries temporais, muitas vezes o objetivo principal é fazer previsões e analisar o comportamento da cópula variando no tempo.

Levantadas todas as questões, são sugeridas então como pesquisas futuras:

- Cálculo das taxas de convergência, considerando uma extensão do estimador proposto para o caso não linear, por meio de threshold;

- Propor um estimador adaptativo, para o qual não seja necessário a escolha do nível ótimo;

- Para o caso de séries temporais, considerar a estimação da cópula via ondaletas, porém variando no tempo, permitindo o cálculo de previsões. 


\section{Apêndice A}

\section{Desigualdades}

\section{A.1 Desigualdade de Dvoretsky-Kiefer-Wolfowitz}

Dadas $X_{1}, X_{2}, \ldots, X_{n}$ variáveis aleatórias reais, independentes e identicamente distribuídas, com função distribuição comum $F($.$) , seja F_{n}($.$) a função distribuição$ empírica associada, dada por

$$
F_{n}(x)=\frac{1}{n} \sum_{i=1}^{n} \mathbb{I}\left\{X_{i} \leq x\right\} \quad x \in \mathbb{R} .
$$

De acordo com Dvoretzky et al. (1956), dado uma constante $\delta>0$,

$$
P\left\{\sup _{x \in \mathbb{R}}\left(F_{n}(x)-F(x)\right)>\delta\right\} \leq \exp \left\{-2 n \delta^{2}\right\}, \text { para cada } \delta \geq \sqrt{\frac{1}{2 n} \ln (2)},
$$

o que implica em

$$
P\left\{\sup _{x \in \mathbb{R}}\left|F_{n}(x)-F(x)\right|>\delta\right\} \leq 2 \exp \left\{-2 n \delta^{2}\right\}, \text { para cada } \delta>0 .
$$

\section{A.2 Desigualdade de Rosenthal}

Como apresentado em Rosenthal (1970), seja $p \leq 2$ e seja $X_{1}, X_{2}, \ldots, X_{n}$ variáveis aleatórias independentes tais que $\mathbb{E}\left(X_{i}\right)=0$ e $\mathbb{E}\left(\left|X_{i}\right|^{p}\right)<\infty$. 
Então, existe $C(p)>0$ tal que

$$
\mathbb{E}\left(\left|\sum_{i=1}^{n} X_{i}\right|^{p}\right) \leq C(p)\left\{\sum_{i=1}^{n} \mathbb{E}\left(\left|X_{i}\right|^{p}\right)+\left(\sum_{i=1}^{n} \mathbb{E}\left(X_{i}^{2}\right)\right)^{\frac{p}{2}}\right\}
$$

\section{A.3 Desigualdade de Davydov}

O método para encontrar um limitante para a Covariância de duas variáveis aleatórias reais $X$ e $Y$, dadas as distribuições marginais e os coeficientes $\alpha$-mixing foi inicialmente estudado por Davydov (1968), e depois detalhado por Rio (1993), em que considerando $r>2$, dado que $\mathbb{E}|X|^{r}, \mathbb{E}|Y|^{r}<\infty$,

$$
|\operatorname{Cov}(X, Y)| \leq 2 \frac{r}{r-2}(2 \alpha)^{1-\frac{2}{r}}\left(\mathbb{E}|X|^{r}\right)^{\frac{1}{r}}\left(\mathbb{E}|Y|^{r}\right)^{\frac{1}{r}} .
$$




\section{Apêndice B}

\section{Ajuste de Modelos}

\section{B.1 Casos de séries temporais}

\section{B.1.1 Séries CAC40 - DAX30}

Os modelos que apresentaram melhores ajustes aos conjuntos de dados foram $\mathrm{AR}(7)$ $\operatorname{GARCH}(1,1)$ para a série $\mathrm{CAC} 40$ e $\mathrm{AR}(6)-\mathrm{GARCH}(1,1)$ para a série DAX30, ambas com alguns coeficientes não significativos aos ajustes. Estes coeficientes foram desconsiderados, como pode ser visto a seguir.

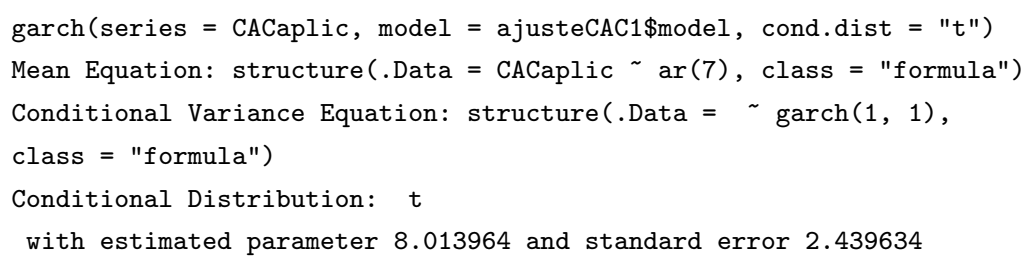

Estimated Coefficients

\begin{tabular}{|c|c|c|c|c|}
\hline & Value & Std.Error & $t$ value & $\operatorname{Pr}(>|t|)$ \\
\hline C & $1.944 \mathrm{e}-003$ & $3.918 e-004$ & 4.963 & $8.144 e-007$ \\
\hline $\operatorname{AR}(1)$ & $0.000 e+000$ & NA & NA & NA \\
\hline $\operatorname{AR}(2)$ & $0.000 e+000$ & NA & NA & NA \\
\hline $\operatorname{AR}(3)$ & $-7.727 e-002$ & $3.143 e-002$ & -2.458 & $1.413 e-002$ \\
\hline $\operatorname{AR}(4)$ & $0.000 e+000$ & NA & NA & NA \\
\hline
\end{tabular}




$\begin{array}{rrrrr}\text { AR (5) } & 0.000 \mathrm{e}+000 & \mathrm{NA} & \mathrm{NA} & \mathrm{NA} \\ \mathrm{AR}(6) & 0.000 \mathrm{e}+000 & \mathrm{NA} & \mathrm{NA} & \mathrm{NA} \\ \mathrm{AR}(7) & -6.300 \mathrm{e}-002 & 3.092 \mathrm{e}-002 & -2.037 & 4.187 \mathrm{e}-002 \\ \mathrm{~A} & 5.959 \mathrm{e}-006 & 2.914 \mathrm{e}-006 & 2.045 & 4.111 \mathrm{e}-002 \\ \mathrm{ARCH}(1) & 6.531 \mathrm{e}-002 & 1.779 \mathrm{e}-002 & 3.672 & 2.536 \mathrm{e}-004 \\ \text { GARCH(1) } & 9.043 \mathrm{e}-001 & 2.642 \mathrm{e}-002 & 34.224 & 0.000 \mathrm{e}+000\end{array}$

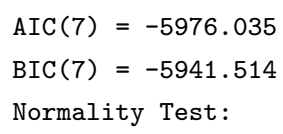

Ljung-Box test for standardized residuals:

Statistic P-value Chi`2-d.f.

$$
\begin{array}{lll}
7.883 & 0.7942 \quad 12
\end{array}
$$

Ljung-Box test for squared standardized residuals:

Statistic P-value Chi^2-d.f.
$\quad 8.5610 .7399 \quad 12$
Lagrange multiplier test:

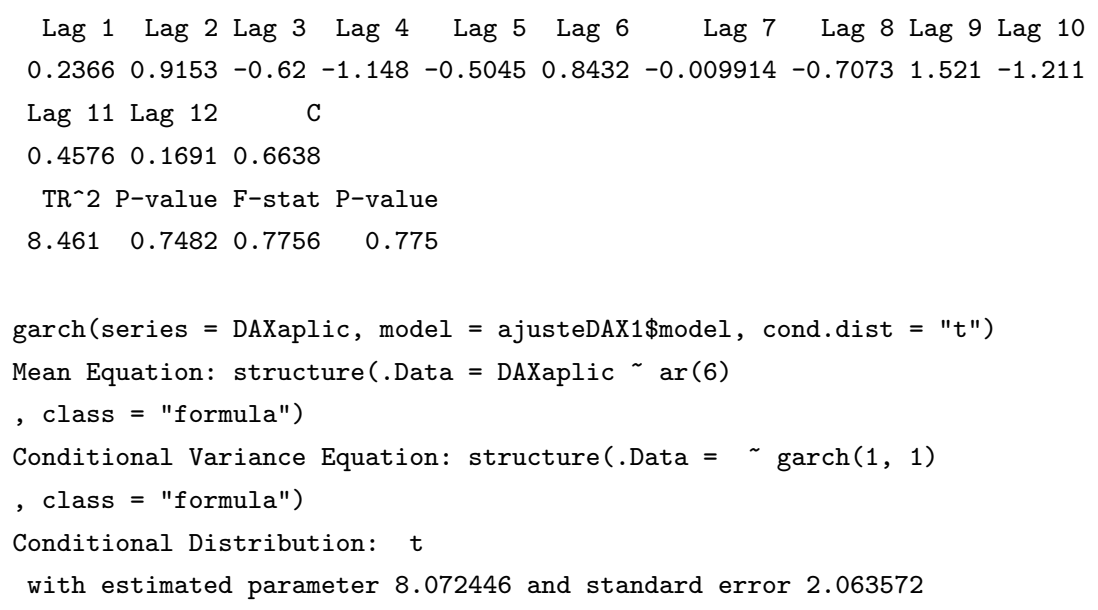




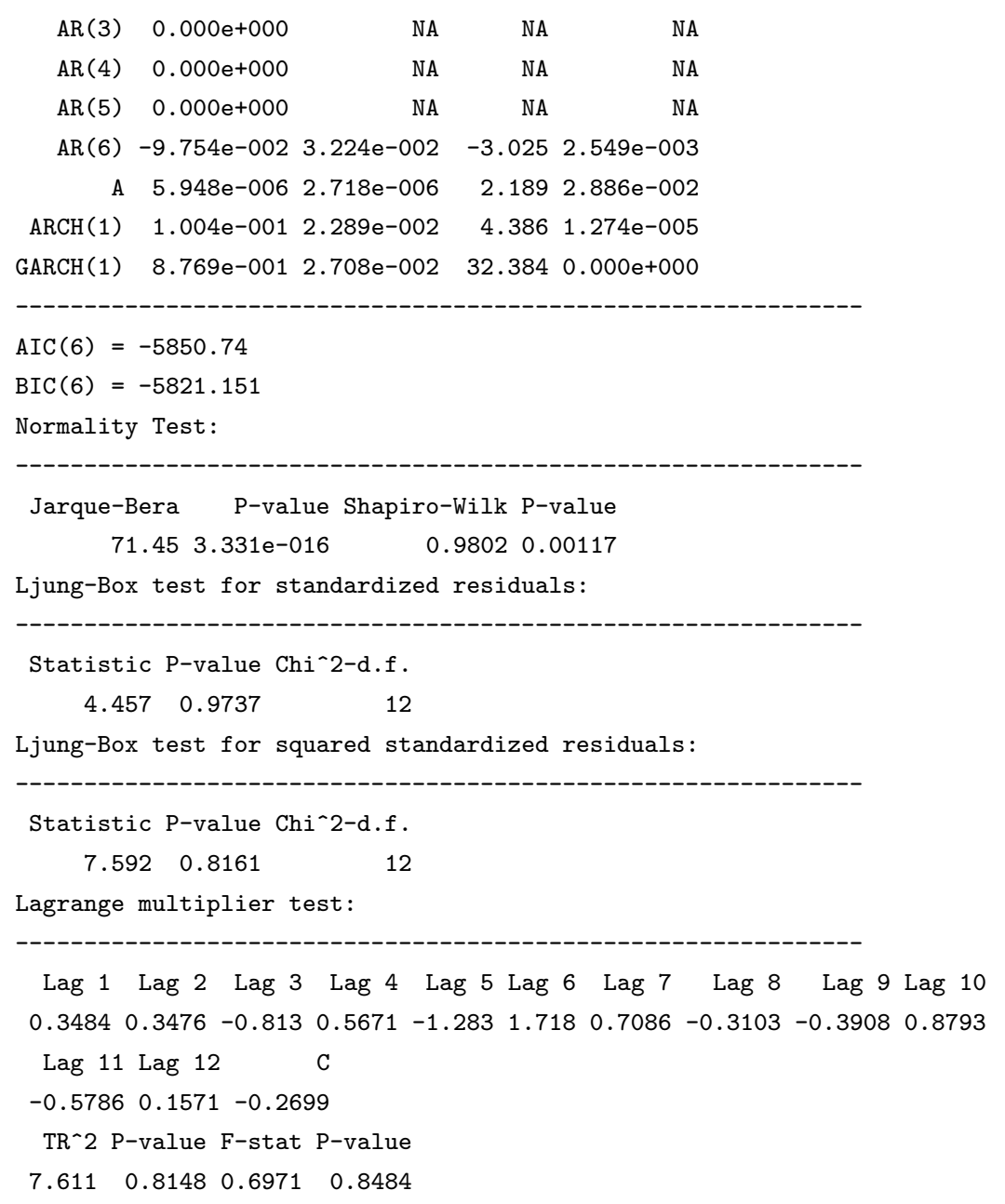

Aos resíduos padronizados dos modelos ARMA-GARCH ajustados, foram ajustadas as cópulas paramétricas, como podem ser vistas a seguir.

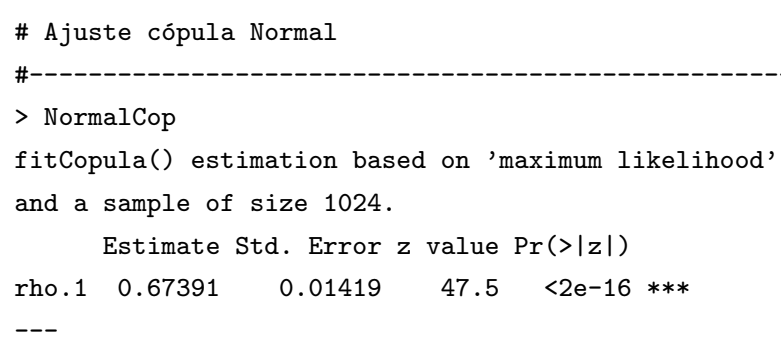




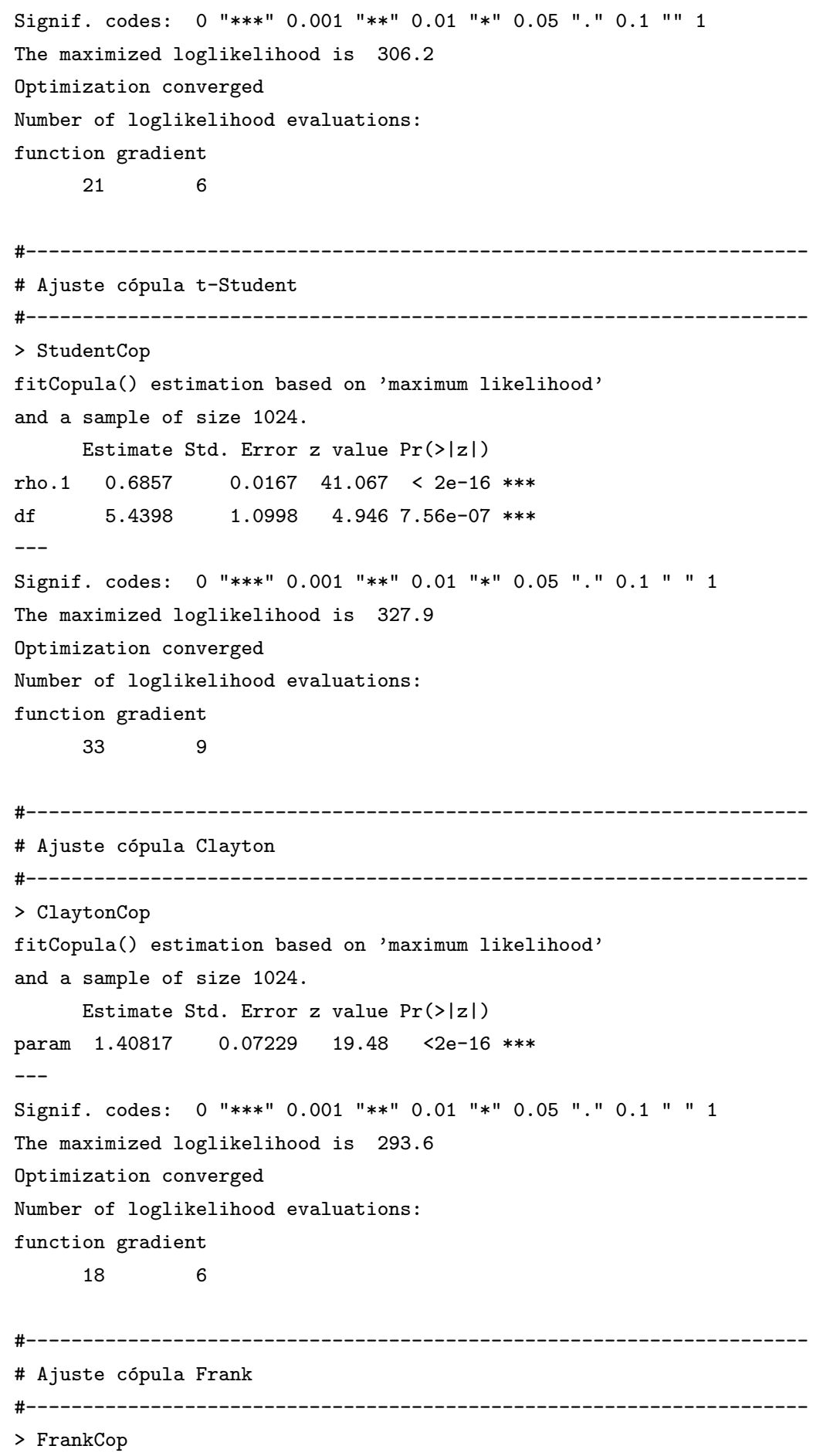




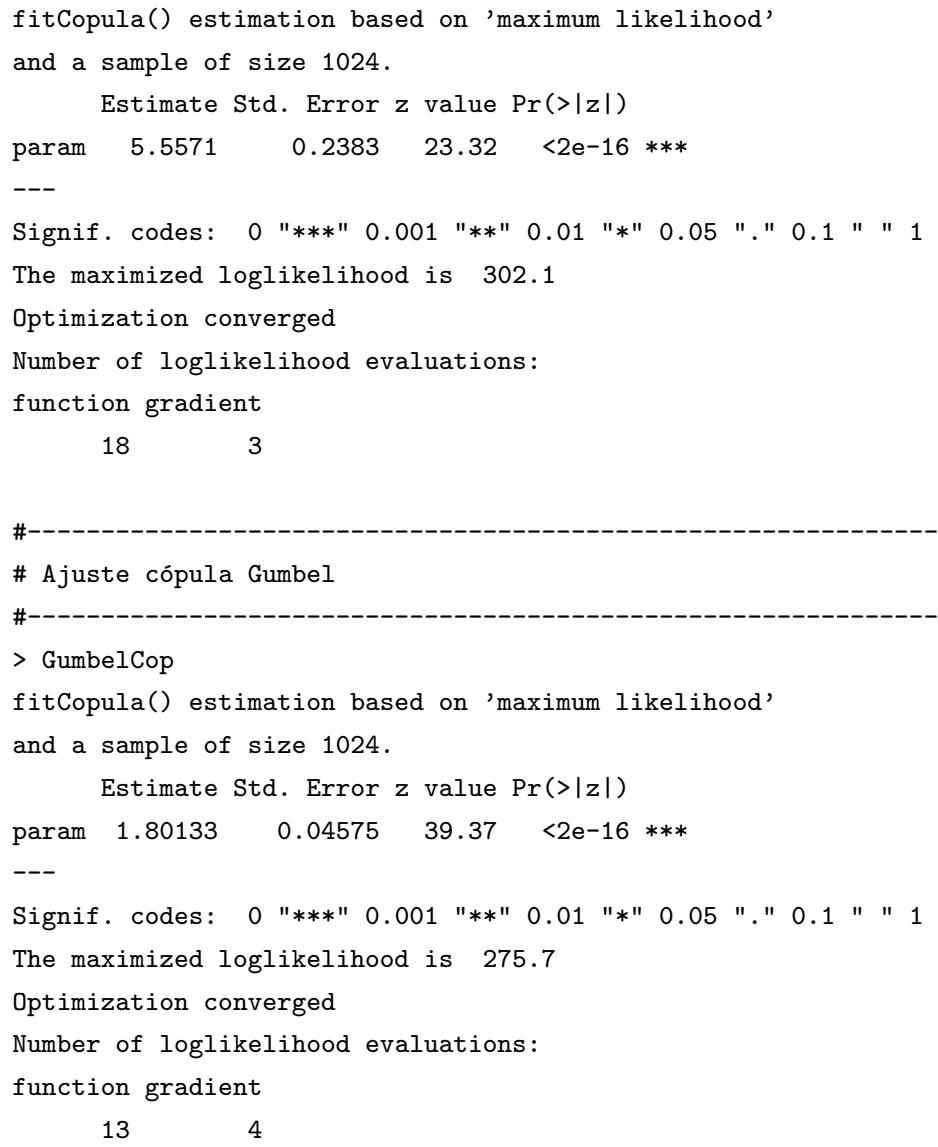

\section{B.1.2 Séries Ibovespa - IPC}

Para a série Ibovespa, o teste de correlação serial e o gráfico de autocorrelação indicaram que não seria necessário ajuste do modelo ARMA-GARCH.

$>$ autocorTest (IBVaplic, method $=$ "lb", na.rm $=\mathrm{T}, \mathrm{lag}=20$ )

Test for Autocorrelation: Ljung-Box

$\mathrm{Null}$ Hypothesis: no autocorrelation

Test Statistics:

Test Stat 30.2412

p.value 0.0660

Dist. under Null: chi-square with 20 degrees of freedom

Total Observ.: 1024 
Para a série IPC, ajustou-se somente o modelo GARCH(1,1). Os resultados para o ajuste são apresentados a seguir.

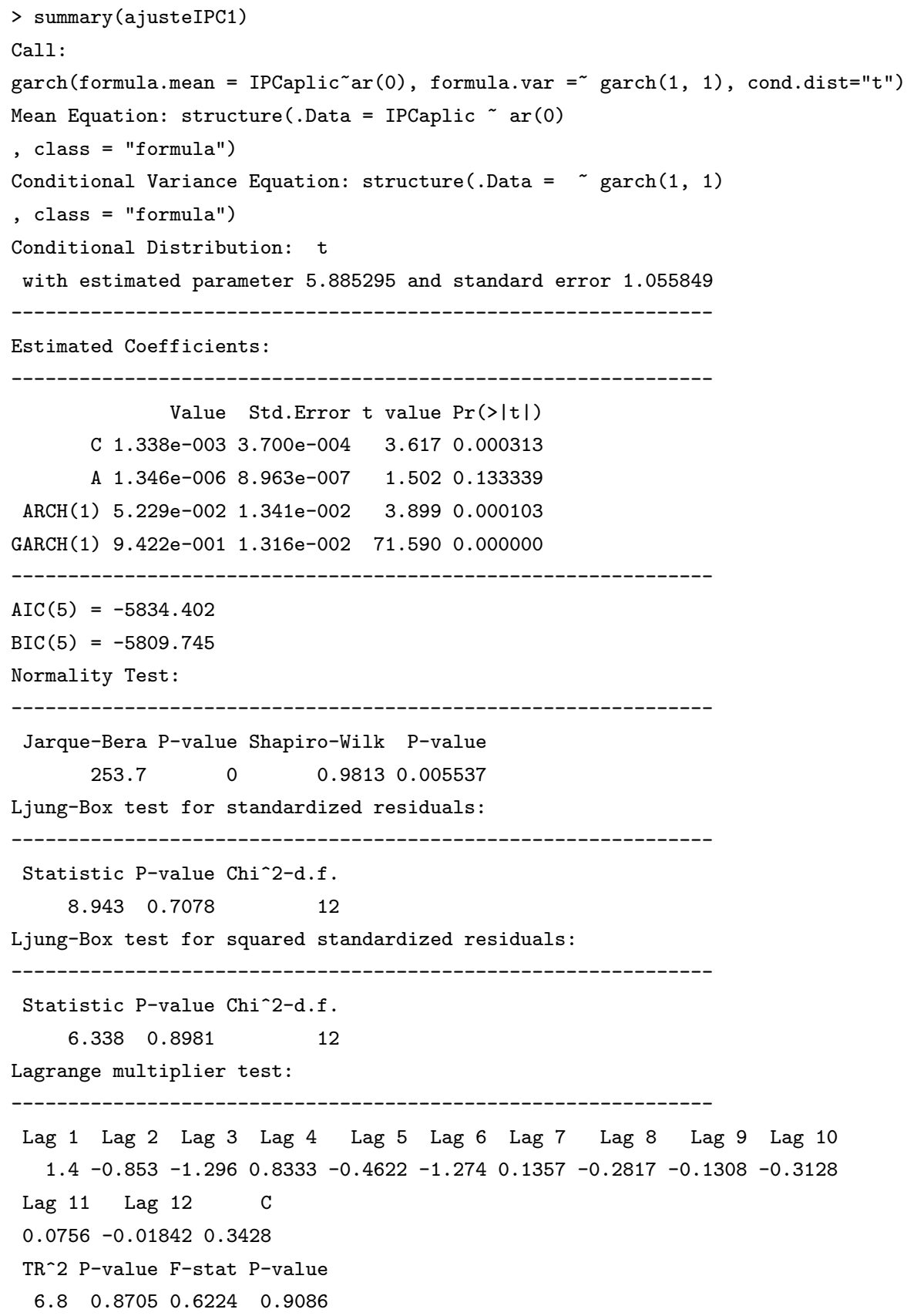


Os valores dos ajustes de cópulas paramétricas para as séries do Ibovespa e IPC também são apresentados a seguir.

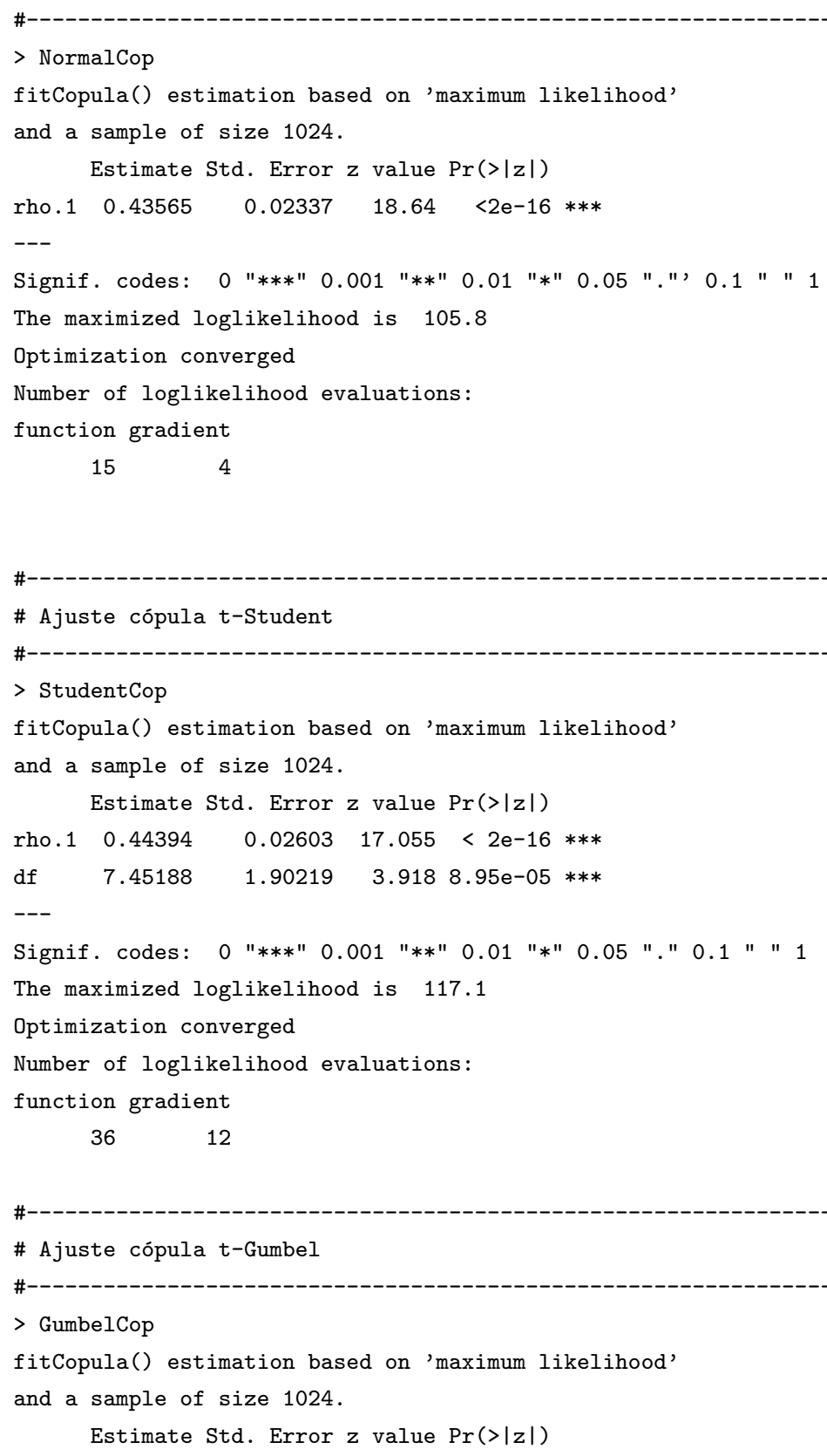




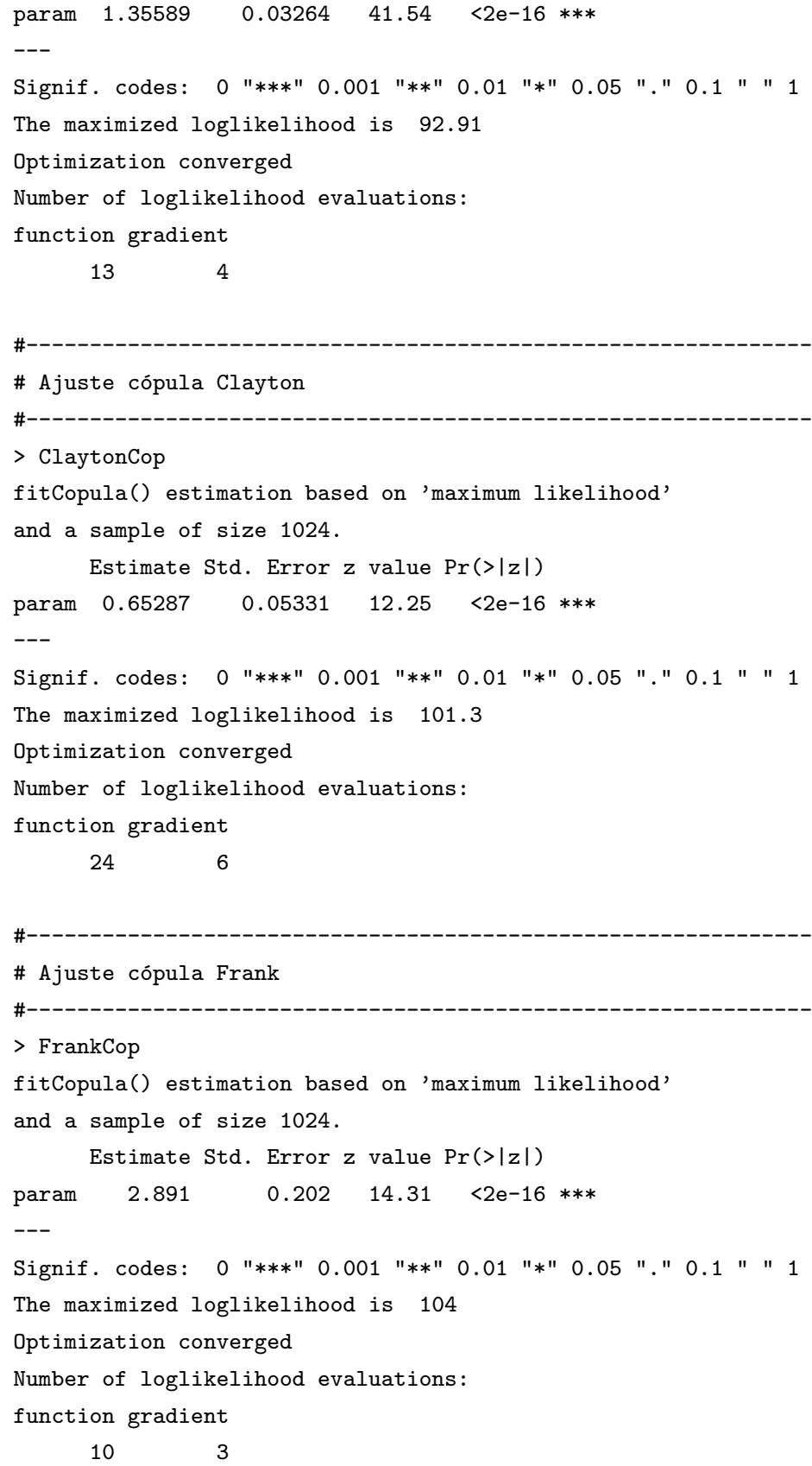




\section{B.1.3 Séries SP500 - DJ}

Foram ajustados os modelos $\mathrm{AR}(7)-\mathrm{GARCH}(1,1)$ para as séries do Ibovespa e IPC, como pode ser visto a seguir.

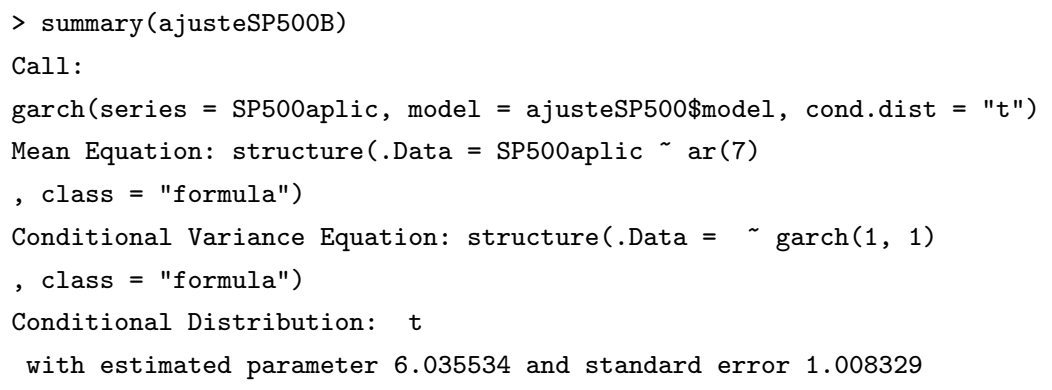


Lagrange multiplier test:

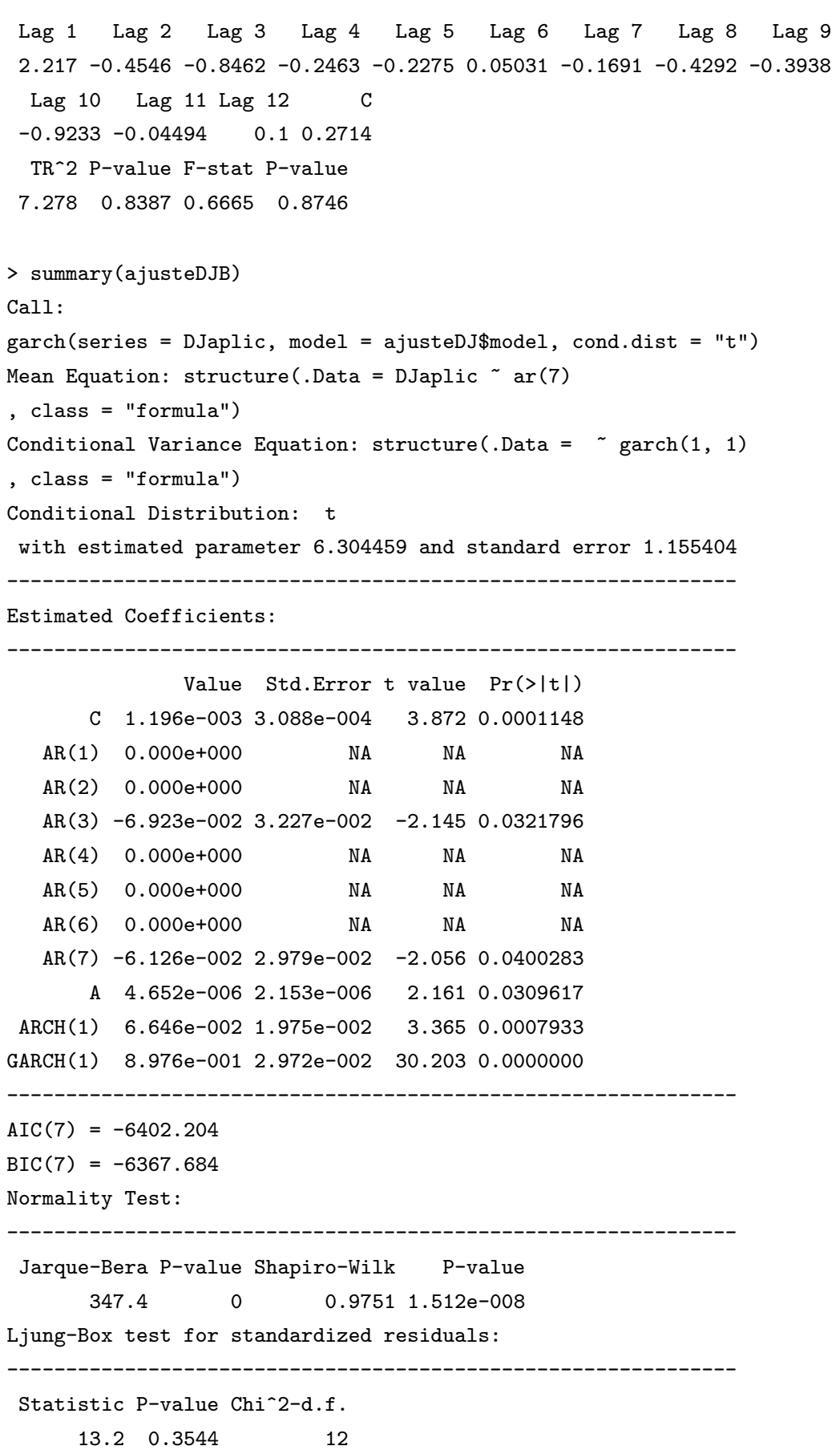


Ljung-Box test for squared standardized residuals:

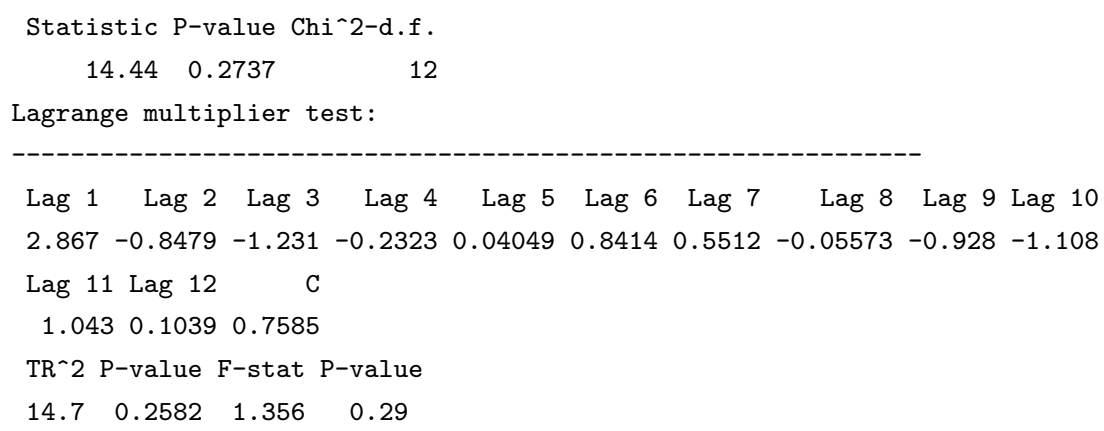

Aos resíduos padronizados, foram ajustadas cópulas paramétricas. Os ajustes são apresentados a seguir.

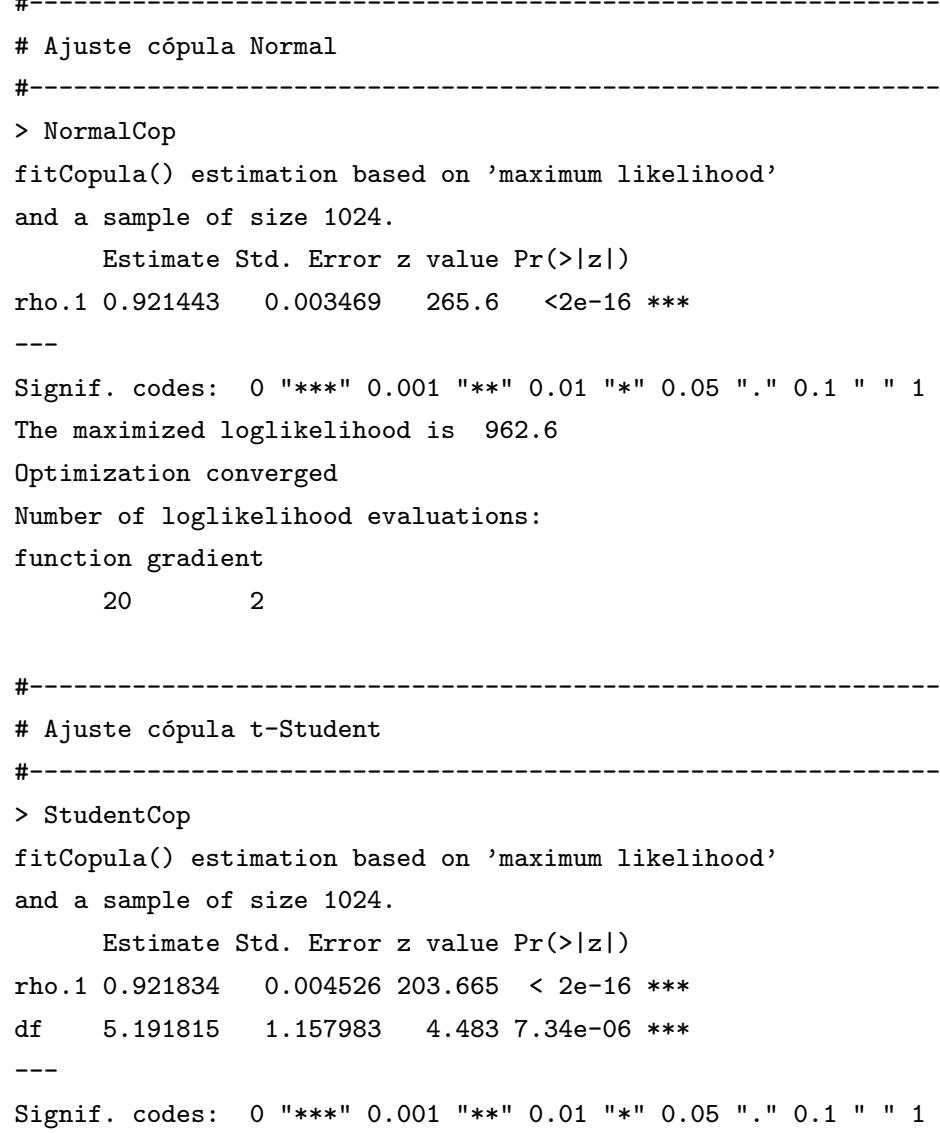


The maximized loglikelihood is 981.8

Optimization converged

Number of loglikelihood evaluations:

function gradient

28

4

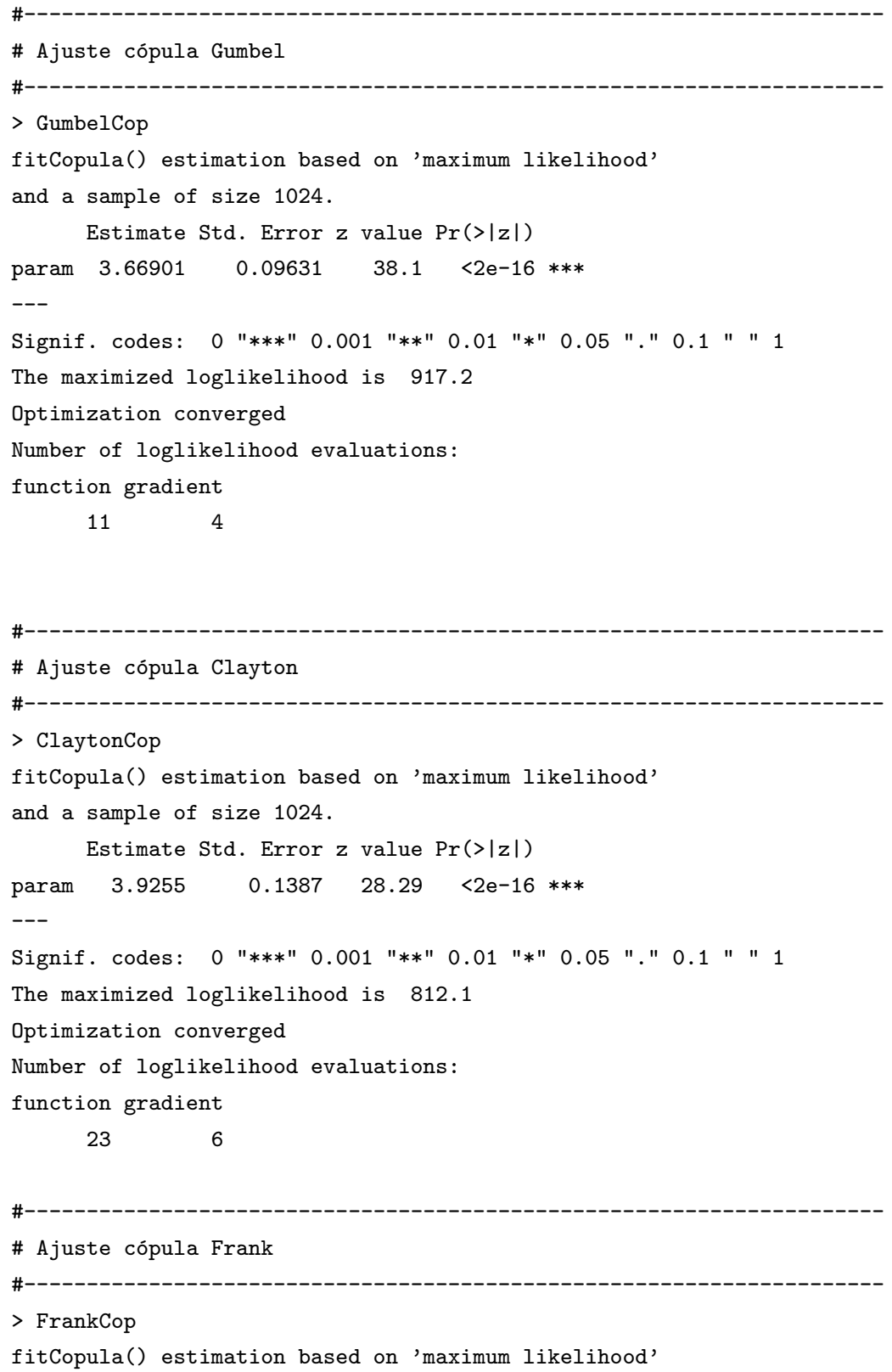




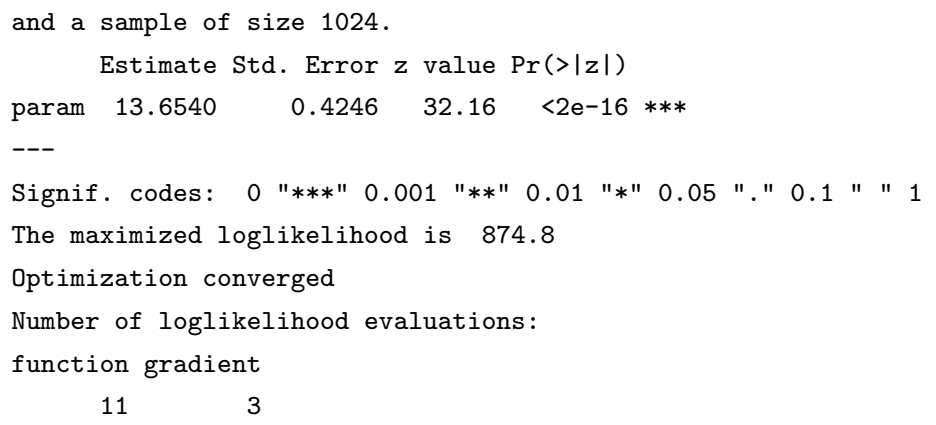

\section{B.2 Caso independente}

\section{B.2.1 Séries Lucro líquido - Margem de vendas}

Considerando os postos padronizados de cada séries, foram feitos ajustes de cópulas paramétricas, como é mostrado a seguir.

\# Ajuste cópula Normal

$>$ NormalCop

fitCopula() estimation based on 'maximum likelihood'

and a sample of size 864 .

Estimate Std. Error $\mathrm{z}$ value $\operatorname{Pr}(>|z|)$

rho.1 $0.833766 \quad 0.007976 \quad 104.5<2 e-16 * *$

$---$

Signif. codes: 0 "***" 0.001 "**" 0.01 "*" 0.05 ". 0.1 " 1

The maximized loglikelihood is 508.4

Optimization converged

Number of loglikelihood evaluations:

function gradient

26

7

\# Ajuste cópula t-Student

\#--

$>$ StudentCop

fitCopula() estimation based on 'maximum likelihood'

and a sample of size 864 .

Estimate Std. Error $\mathrm{z}$ value $\operatorname{Pr}(>|z|)$ 


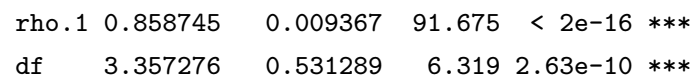




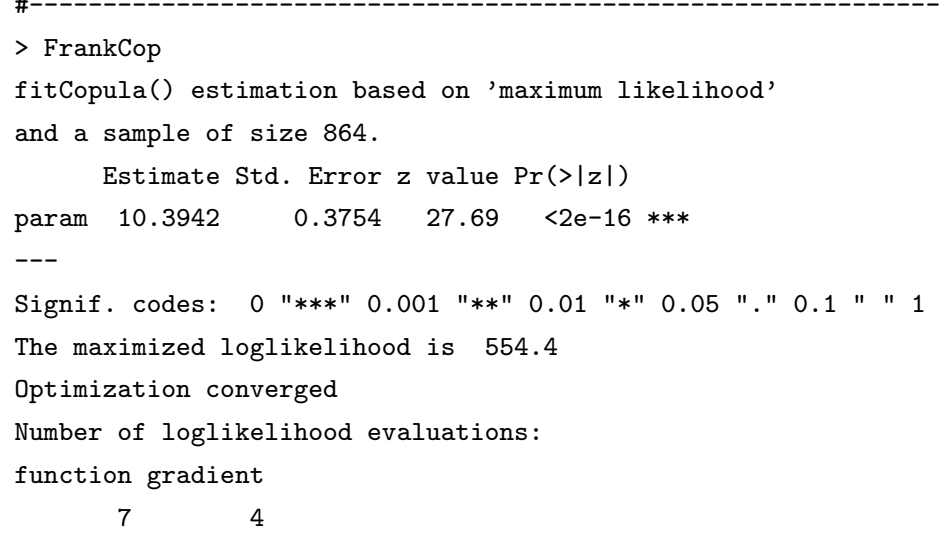




\section{Referências Bibliográficas}

Autin, F., Le Pennec and Tribouley, K. (2010), Thresholding methods to estimate copula density, Journal of Multivariate Analysis, 101, 200-222.

Boggess, A., Narcowich, F. J. (2001), A First course in wavelets with fourier analysis, Texas, Prentice Hall.

Cohen, A., Daubechies, I. and Vial, P. (1993), Wavelets on the interval and fast wavelet transforms, Applied and Computational Harmonic Analysis, 1, 54-81.

Doukhan, P. (1995). Mixing, Lecture Notes in Statistics, 85. New York: SpringerVerlag.

Davydov, Y. A.(1968). Convergence of distributions generated by stationary stochastic processes, Theory of Probability \& Its Applications , 13 (4), 691-696.

Dvoretzky, A., Kiefer, J. and Wolfowitz, J. (1956), Asymptotic minimax character of the sample distribution function and of the classical multinomial estimator, Annals of Mathematical Statistics, 27 (3): 642-669, doi:10.1214/aoms/1177728174, MR 0083864 .

Fermanian, J.-D. and Scaillet, O. (2003), Nonparametric estimation of copulas for time series, Journal of Risk, 5, 25-54.

Genest, C., Massiello, E. and Tribouley, K. (2009), Estimating copula densities through wavelets, Insurance: Mathematics and Economics, 44, 170-181. 
Härdle, W., Kerkyacharian, G., Picard, D. and Tsybakov, A. (1998), Wavelets, Approximation, and Statistical Applications, Lecture Notes in Statistics, 129. New York: Springer.

Latif, S.A., Morettin, P.A. (2010), Introdução a cópulas e aplicações na avaliação do desempenho de empresas, Revista Brasileira de Estatística, vol 71, 234, 121-148.

Mallat, S. (1989a), Multiresolution approximations and wavelet orthonormal bases of $L_{2}(\mathbb{R})$, Transactions of the American Mathematical Society, 315, 69-87.

Mallat, S. (1989b), A theory for multiresolution sinal decomposition: Wavelets representation, IEEE e Transictions on Pattern Analysis and Machine Intelligence, 11, 674-692.

Mendes, B. V. de M. (2005), Computing conditional VaR using time-varying copulas, Brazilian Review of Finance, 3, 251-265.

Meyer, Y. (1992), Wavelets and Operators, In: Cambridge Studies in Advanced Mathematics, vol 37, Cambridge University Press, Cambridge.

Morettin, P.A. (2014), Ondas e Ondaletas, $2^{\mathrm{a}}$ Edição, São Paulo, Editora da Universidade de São Paulo.

Morettin, P.A., Toloi, C. M. C., Chiann, C., e de Miranda, J. C. S. (2010), Wavelet smoothed empirical copula estimators, Brazilian Review of Finance, 8, 263-281.

Morettin, P.A., Toloi, C. M. C., Chiann, C., e de Miranda, J. C. S. (2011), Wavelet estimation of copulas for time series, Journal of Times Series Econometrics, vol. 3 , Issue 4, 1-29.

Morettin, P.A.(2011), Econometria Financeira, 2a Edição, São Paulo, Editora Blucher.

Nelsen, R. (2005), An Introduction to Copulas, 2nd Ed., Springer, New York. 
Rio, E. (1993), Covariance inequalities for strongly mixing processes, Annales de l'Institut Henri Poincaré, 29, 587-597.

Rosenthal, H.P. (1970), On the subspaces of $L^{p}(p>2)$ spanned by sequences of independent random variables, Israel Journal of Mathematics, 8, 273-303.

Schmidt, R. and Stadtmüller, U. (2006), Non-parametric estimation of tail dependence, Scandinavian Journal of Statistics, 68, 56-57.

Strang, G. and Nguyen, T. (1996), Wavelets and Filter Banks,.

Seeley, R. T. (1961), Fubini implies Leibniz implies $F_{y x}=F_{x y}$, The American Mathematical Monthly, 68, 56-57.

Yu H. (1993), A Glivenko-Cantelli lemma and weak convergence for empirical processes of associated sequences, Probability Theory and Related Fields, 95, 357-370. 